Annales Henri Lebesgue

1 (2018) 1-46

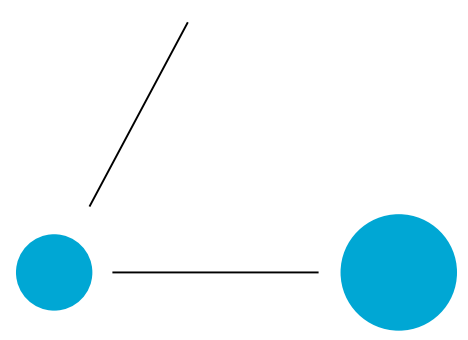

A N N A L E S

HENRI LEBESGUE

HAJER BAHOURI

JEAN - YVES CHEMIN

RAPHAËL DANCHIN

\title{
TEMPERED DISTRIBUTIONS AND FOURIER TRANSFORM ON THE HEISENBERG GROUP
}

DISTRIBUTIONS TEMPÉRÉES ET

TRANSFORMATION DE FOURIER SUR LE GROUPE DE HEISENBERG

Abstract. - We aim at extending the Fourier transform on the Heisenberg group $\mathbb{H}^{d}$, to tempered distributions. Our motivation is to provide the reader with a hands-on approach that allows for further investigating Fourier analysis and PDEs on $\mathbb{H}^{d}$.

As in the Euclidean setting, the strategy is to show that the Fourier transform is an isomorphism on the Schwartz space, then to define the extension by duality. To achieve it, the Fourier transform of an integrable function is viewed as a uniformly continuous mapping on the set $\widetilde{\mathbb{H}}^{d}=\mathbb{N}^{d} \times \mathbb{N}^{d} \times \mathbb{R} \backslash\{0\}$, that may be completed to a larger set $\widetilde{\mathbb{H}}^{d}$ for some suitable distance. This viewpoint provides a user friendly description of the range of the Schwartz space on $\mathbb{H}^{d}$ by the Fourier transform, which makes the extension to the whole set of tempered distributions straightforward.

Keywords: Fourier transform, Heisenberg group, frequency space, tempered distributions, Schwartz space.

2010 Mathematics Subject Classification: 43A30, 43A80.

DOI: https://doi.org/10.5802/ahl.1

(*) The authors are deeply indebted to the anonymous referees for their very complete reading of the manuscript. Their long list of remarks and constructive suggestions greatly contributed to improve the final version of this work. 
To highlight the strength of our approach, we give examples of computations of Fourier transforms of tempered distributions that do not correspond to integrable or square integrable functions. The most striking one is a formula for the Fourier transform of functions on $\mathbb{H}^{d}$ that are independent of the vertical variable, an open question, to the best of our knowledge.

RÉsumé. - Dans cet article, on s'intéresse à l'extension de la transformation de Fourier sur le groupe de Heisenberg $\mathbb{H}^{d}$, aux distributions tempérées. Notre but est de donner au lecteur un cadre adapté à l'étude de l'analyse de Fourier et des EDP sur $\mathbb{H}^{d}$.

Comme dans $\mathbb{R}^{n}$, il s'agit en premier lieu de montrer que la transformation de Fourier est un isomorphisme sur l'espace de Schwartz, puis d'étendre sa définition par dualité aux distributions tempérées. Pour ce faire, on définit la transformée de Fourier d'une fonction intégrable comme étant une fonction uniformément continue sur l'ensemble $\widetilde{\mathbb{H}}^{d}=\mathbb{N}^{d} \times \mathbb{N}^{d} \times \mathbb{R} \backslash\{0\}$, qui peut être complété en un ensemble $\widetilde{\mathbb{H}}^{d}$ pour une distance adéquate. Ce point de vue donne une caractérisation simple de l'image de l'espace de Schwartz sur $\mathbb{H}^{d}$ par la transformation de Fourier, permettant ainsi d'étendre sa définition à l'ensemble des distributions tempérées.

Pour illustrer la puissance de notre approche, on donne quelques exemples de calculs explicites de transformées de Fourier de fonctions ou distributions tempérées qui ne correspondent pas à des fonctions intégrables ou de carrés intégrables. Le plus spectaculaire est l'obtention d'une formule explicite pour la transformée de Fourier de fonctions régulières indépendantes de la variable verticale. Pour répondre à cette question de façon simple, avoir pris le soin de le compléter au préalable l'espace des fréquences $\widetilde{\mathbb{H}}^{d}$ s'avère fondamental.

\section{Introduction}

The present work aims at extending Fourier analysis on the Heisenberg group to tempered distributions. It is by now very classical that in the case of a commutative group, the Fourier transform is a function on the group of characters. In the Euclidean space $\mathbb{R}^{n}$ the group of characters may be identified with the dual space $\left(\mathbb{R}^{n}\right)^{\star}$ of $\mathbb{R}^{n}$ through the map $\xi \mapsto e^{i\langle\xi, \cdot\rangle}$, where $\langle\xi, \cdot\rangle$ designate the value of the one-form $\xi$ when applied to elements of $\mathbb{R}^{n}$, and the Fourier transform of an integrable function $f$ may be seen as the function on $\left(\mathbb{R}^{n}\right)^{\star}$, defined by

$$
\mathcal{F}(f)(\xi)=\widehat{f}(\xi) \stackrel{\text { def }}{=} \int_{\mathbb{R}^{n}} e^{-i\langle\xi, x\rangle} f(x) \mathrm{d} x .
$$

A fundamental fact of the distribution theory on $\mathbb{R}^{n}$ is that the Fourier transform is a bi-continuous isomorphism on the Schwartz space $\mathcal{S}\left(\mathbb{R}^{n}\right)$ - the set of smooth functions whose derivatives decay at infinity faster than any power. Hence, one can define the transposed Fourier transform ${ }^{t} \mathcal{F}$ on the so-called set of tempered distributions $\mathcal{S}^{\prime}\left(\mathbb{R}^{n}\right)$, that is the topological dual of $\mathcal{S}\left(\mathbb{R}^{n}\right)$ (see e.g. [Rud62, Sch98] for a self-contained presentation). As the whole distribution theory on $\mathbb{R}^{n}$ is based on identifying locally integrable functions with linear forms by means of the Lebesgue integral, one can look for a more direct relationship between ${ }^{t} \mathcal{F}$ and $\mathcal{F}$, by considering the following bilinear form on $\mathcal{S}\left(\mathbb{R}^{n}\right) \times \mathcal{S}\left(\mathbb{R}^{n}\right)$ :

$$
\mathcal{B}_{\mathbb{R}}(f, \phi) \stackrel{\text { def }}{=} \int_{T^{\star} \mathbb{R}^{n}} f(x) e^{-i\langle\xi, x\rangle} \phi(\xi) \mathrm{d} x \mathrm{~d} \xi,
$$

where the cotangent bundle $T^{\star} \mathbb{R}^{n}$ of $\mathbb{R}^{n}$ is identified with $\mathbb{R}^{n} \times\left(\mathbb{R}^{n}\right)^{\star}$. This allows to identify ${ }^{t} \mathcal{F}_{\mid \mathcal{S}\left(\left(\mathbb{R}^{n}\right)^{\star}\right)}$ with $\mathcal{F}_{\mid \mathcal{S}\left(\mathbb{R}^{n}\right)}$, and it is thus natural to define the extension of 
$\mathcal{F}$ on $\mathcal{S}^{\prime}\left(\mathbb{R}^{n}\right)$ to be ${ }^{t} \mathcal{F}$, that is,

$$
\forall(T, \phi) \in \mathcal{S}^{\prime}\left(\mathbb{R}^{n}\right) \times \mathcal{S}\left(\mathbb{R}^{n}\right),\langle\mathcal{F} T, \phi\rangle_{\mathcal{S}^{\prime}\left(\mathbb{R}^{n}\right) \times \mathcal{S}\left(\mathbb{R}^{n}\right)} \stackrel{\text { def }}{=}\langle T, \mathcal{F} \phi\rangle_{\mathcal{S}^{\prime}\left(\mathbb{R}^{n}\right) \times \mathcal{S}\left(\mathbb{R}^{n}\right)} .
$$

We aim at implementing that procedure on the Heisenberg group $\mathbb{H}^{d}$. As in the Euclidean case, to achieve our goal, it is fundamental to have a handy characterization of the range of the Schwartz space on $\mathbb{H}^{d}$ by the Fourier transform. The first attempt in that direction goes back to the pioneering works by D. Geller in [Gel77, Gel80] (see also [ADBR13, Far10, LT14] and the references therein). However, the description of $\mathcal{F}\left(\mathcal{S}\left(\mathbb{H}^{d}\right)\right)$ that is given therein relies on complicated asymptotic series, so that whether it allows to extend the Fourier transform to tempered distribution is unclear.

We here aim at presenting a rather elementary description of $\mathcal{F}\left(\mathcal{S}\left(\mathbb{H}^{d}\right)\right)$ that is based on the approach that we developed recently in $[\mathrm{BCD}]$. Our motivation is threefold. First, our approach enables us to recover easily different classical but highly nontrivial results like the fact that the fundamental solution of the heat flow or kernels of multipliers of the form $a\left(-\Delta_{\mathbb{H}}\right)$ with $a \in \mathcal{S}(\mathbb{R})$, belong to $\mathcal{S}\left(\mathbb{H}^{d}\right)$. Second, we are able to compute explicitly the Fourier transform of a number of functions which are not integrable like, for instance, that of functions that are independent of the vertical variable. Third, having a complete description of both $\mathcal{F}\left(\mathcal{S}\left(\mathbb{H}^{d}\right)\right)$ and $\mathcal{F}\left(\mathcal{S}^{\prime}\left(\mathbb{H}^{d}\right)\right)$ gives us a solid basis to tackle more complicated Fourier analysis issues, with applications to Partial Differential Equations.

At this stage of the paper, we have to recall the definitions of the Heisenberg group $\mathbb{H}^{d}$ and of the Fourier transform on $\mathbb{H}^{d}$, and a few basic properties. We shall define $\mathbb{H}^{d}$ to be the set $T^{\star} \mathbb{R}^{d} \times \mathbb{R}$ equipped with the product law

$$
w \cdot w^{\prime} \stackrel{\text { def }}{=}\left(Y+Y^{\prime}, s+s^{\prime}+2 \sigma\left(Y, Y^{\prime}\right)\right)=\left(y+y^{\prime}, \eta+\eta^{\prime}, s+s^{\prime}+2\left\langle\eta, y^{\prime}\right\rangle-2\left\langle\eta^{\prime}, y\right\rangle\right)
$$

where $w=(Y, s)=(y, \eta, s)$ and $w^{\prime}=\left(Y^{\prime}, s^{\prime}\right)=\left(y^{\prime}, \eta^{\prime}, s^{\prime}\right)$ are generic elements of $\mathbb{H}^{d}$. In the above definition, the notation $\langle\cdot, \cdot\rangle$ designates the duality bracket between $\left(\mathbb{R}^{d}\right)^{\star}$ and $\mathbb{R}^{d}$ and $\sigma$ is the canonical symplectic form on $\mathbb{R}^{2 d}$, seen as $T^{\star} \mathbb{R}^{d}$. This gives $\mathbb{H}^{d}$ the structure of a non commutative group for which $w^{-1}=-w$. The reader will find more details in the books [BFKG12, FH84, FR14, Fol89, FS82, Ste93, Tay86, Tha98] and in the references therein.

In accordance with the above product formula, one can define the set of the dilations on the Heisenberg group to be the family of operators $\left(\delta_{a}\right)_{a>0}$ given by

$$
\delta_{a}(w)=\delta_{a}(Y, s) \stackrel{\text { def }}{=}\left(a Y, a^{2} s\right) .
$$

Note that dilations commute with the product law on $\mathbb{H}^{d}$, that is $\delta_{a}\left(w \cdot w^{\prime}\right)=$ $\delta_{a}(w) \cdot \delta_{a}\left(w^{\prime}\right)$. Furthermore, as the determinant of $\delta_{a}$ (seen as an automorphism of $\left.\mathbb{R}^{2 d+1}\right)$ is $a^{2 d+2}$, the homogeneous dimension of $\mathbb{H}^{d}$ is $N \stackrel{\text { def }}{=} 2 d+2$.

The Heisenberg group is endowed with a smooth left invariant Haar measure, which, in the coordinate system $(y, \eta, s)$ is just the Lebesgue measure on $\mathbb{R}^{2 d+1}$. The corresponding Lebesgue spaces $L^{p}\left(\mathbb{H}^{d}\right)$ are thus the sets of measurable functions $f: \mathbb{H}^{d} \rightarrow \mathbb{C}$ such that

$$
\|f\|_{L^{p}\left(\mathbb{H}^{d}\right)} \stackrel{\text { def }}{=}\left(\int_{\mathbb{H}^{d}}|f(w)|^{p} \mathrm{~d} w\right)^{\frac{1}{p}}<\infty, \quad \text { if } 1 \leqslant p<\infty,
$$

with the standard modification if $p=\infty$. 
The convolution product of any two integrable functions $f$ and $g$ is given by

$$
f \star g(w) \stackrel{\text { def }}{=} \int_{\mathbb{H}^{d}} f\left(w \cdot v^{-1}\right) g(v) \mathrm{d} v=\int_{\mathbb{H}^{d}} f(v) g\left(v^{-1} \cdot w\right) \mathrm{d} v .
$$

As in the Euclidean case, the convolution product is an associative binary operation on the set of integrable functions. Even though it is no longer commutative, the following Young inequalities hold true:

$$
\|f \star g\|_{L^{r}} \leqslant\|f\|_{L^{p}}\|g\|_{L^{q}}, \quad \text { whenever } 1 \leqslant p, q, r \leqslant \infty \text { and } \frac{1}{r}=\frac{1}{p}+\frac{1}{q}-1 .
$$

The Schwartz space $\mathcal{S}\left(\mathbb{H}^{d}\right)$ corresponds to the Schwartz space $\mathcal{S}\left(\mathbb{R}^{2 d+1}\right.$ ) (an equivalent definition involving the Heisenberg structure will be provided in Appendix A.3).

As the Heisenberg group is noncommutative, defining the Fourier transform of integrable functions on $\mathbb{H}^{d}$, by a formula similar to (1.1) is no longer relevant. One has to use a more elaborate family of irreducible representations of $\mathbb{H}^{d}$, all of them being unitary equivalent (see e.g. [Tay86, Chapter 2]) to the Schrödinger representation $\left(U^{\lambda}\right)_{\lambda \in \mathbb{R} \backslash\{0\}}$ which is the family of group homomorphisms $w \mapsto U_{w}^{\lambda}$ between $\mathbb{H}^{d}$ and the unitary group $\mathcal{U}\left(L^{2}\left(\mathbb{R}^{d}\right)\right)$ of $L^{2}\left(\mathbb{R}^{d}\right)$ defined for all $w=(y, \eta, s)$ in $\mathbb{H}^{d}$ and $u$ in $L^{2}\left(\mathbb{R}^{d}\right)$ by

$$
U_{w}^{\lambda} u(x) \stackrel{\text { def }}{=} e^{-i \lambda(s+2\langle\eta, x-y\rangle)} u(x-2 y) .
$$

The standard definition of the Fourier transform then reads as follows.

Definition 1.1. - For $f$ in $L^{1}\left(\mathbb{H}^{d}\right)$ and $\lambda$ in $\mathbb{R} \backslash\{0\}$, we define

$$
\mathfrak{F}_{\mathbb{H}}(f)(\lambda) \stackrel{\text { def }}{=} \int_{\mathbb{H}^{d}} f(w) U_{w}^{\lambda} \mathrm{d} w .
$$

The function $\mathfrak{F}_{\mathbb{H}}(f)$ which takes values in the space of bounded operators on $L^{2}\left(\mathbb{R}^{d}\right)$ is, by definition, the Fourier transform of $f$.

An obvious drawback of Definition 1.1 is that $\mathfrak{F}_{\mathbb{H}} f$ is not a complex valued function on some "frequency space", but a much more complicated object. Consequently, one can hardly expect to have a simple characterization of the range of the Schwartz space by $\mathfrak{F}_{\mathbb{H}}$, allowing for our extending the Fourier transform to tempered distributions. To overcome that difficulty, we proposed in our recent paper[BCD] an alternative (equivalent) definition that makes the Fourier transform of any integrable function on $\mathbb{H}^{d}$, a continuous function on another (explicit) set $\widehat{\mathbb{H}}^{d}$ endowed with some distance $\widehat{d}$.

Before giving our definition, we need to introduce some notation. Let us first recall that the Lie algebra of left invariant vector fields on $\mathbb{H}^{d}$, that is vector fields commuting with any left translation $\tau_{w}\left(w^{\prime}\right) \stackrel{\text { def }}{=} w \cdot w^{\prime}$, is spanned by the vector fields

$$
S \stackrel{\text { def }}{=} \partial_{s}, \quad \mathcal{X}_{j} \stackrel{\text { def }}{=} \partial_{y_{j}}+2 \eta_{j} \partial_{s} \quad \text { and } \quad \Xi_{j} \stackrel{\text { def }}{=} \partial_{\eta_{j}}-2 y_{j} \partial_{s}, 1 \leqslant j \leqslant d .
$$

The sublaplacian associated to the vector fields $\left(\mathcal{X}_{j}\right)_{1 \leqslant j \leqslant d}$ and $\left(\Xi_{j}\right)_{1 \leqslant j \leqslant d}$ is defined by

$$
\Delta_{\mathbb{H}} \stackrel{\text { def }}{=} \sum_{j=1}^{d}\left(\mathcal{X}_{j}^{2}+\Xi_{j}^{2}\right),
$$


and may be rewritten in terms of the usual derivatives as follows:

$$
\Delta_{\mathbb{H}} f(Y, s)=\Delta_{Y} f(Y, s)+4 \sum_{j=1}^{d}\left(\eta_{j} \partial_{y_{j}}-y_{j} \partial_{\eta_{j}}\right) \partial_{s} f(Y, s)+4|Y|^{2} \partial_{s}^{2} f(Y, s) .
$$

The sublaplacian plays a fundamental role in the Fourier theory of the Heisenberg group. The starting point is the following relation that holds true for all functions $f$ in the Schwartz space (see e.g.[Hue76, Olv74]):

$$
\mathfrak{F}_{\mathbb{H}}\left(\Delta_{\mathbb{H}} f\right)(\lambda)=4 \mathfrak{F}_{\mathbb{H}}(f)(\lambda) \circ \Delta_{\text {osc }}^{\lambda} \quad \text { with } \quad \Delta_{\text {osc }}^{\lambda} u(x) \stackrel{\text { def }}{=} \sum_{j=1}^{d} \partial_{j}^{2} u(x)-\lambda^{2}|x|^{2} u(x) .
$$

In order to take advantage of the spectral structure of the harmonic oscillator, we introduce the corresponding eigenvectors, that is the family of Hermite functions $\left(H_{n}\right)_{n \in \mathbb{N}^{d}}$ defined by

$$
H_{n} \stackrel{\text { def }}{=}\left(\frac{1}{2^{|n|} n !}\right)^{\frac{1}{2}} C^{n} H_{0} \quad \text { with } \quad C^{n} \stackrel{\text { def }}{=} \prod_{j=1}^{d} C_{j}^{n_{j}} \quad \text { and } \quad H_{0}(x) \stackrel{\text { def }}{=} \pi^{-\frac{d}{2}} e^{-\frac{|x|^{2}}{2}}
$$

where $C_{j} \stackrel{\text { def }}{=}-\partial_{j}+M_{j}$ stands for the creation operator with respect to the $j$-th variable and $M_{j}$ is the multiplication operator defined by $M_{j} u(x) \stackrel{\text { def }}{=} x_{j} u(x)$.

As usual, for any multi-index $n$ in $\mathbb{N}^{d}, n ! \stackrel{\text { def }}{=} n_{1} ! \ldots n_{d}$ ! and $|n| \stackrel{\text { def }}{=} n_{1}+\ldots+n_{d}$ stands for the length of $n$ (not to be confused with the Euclidean norm that we shall sometimes denote in the same way for elements of $\mathbb{R}^{d}$ ).

Recall that $\left(H_{n}\right)_{n \in \mathbb{N}^{d}}$ is an orthonormal basis of $L^{2}\left(\mathbb{R}^{d}\right)$, and that we have

$$
\left(-\partial_{j}^{2}+M_{j}^{2}\right) H_{n}=\left(2 n_{j}+1\right) H_{n} \quad \text { and thus } \quad-\Delta_{\text {osc }}^{1} H_{n}=(2|n|+d) H_{n} .
$$

For $\lambda$ in $\mathbb{R} \backslash\{0\}$, we finally introduce the rescaled Hermite function $H_{n, \lambda}(x) \stackrel{\text { def }}{=}$ $|\lambda|^{\frac{d}{4}} H_{n}\left(|\lambda|^{\frac{1}{2}} x\right)$. It is obvious that $\left(H_{n, \lambda}\right)_{n \in \mathbb{N}^{d}}$ is still an orthonormal basis of $L^{2}\left(\mathbb{R}^{d}\right)$ and that

$$
\left(-\partial_{j}^{2}+\lambda^{2} M_{j}^{2}\right) H_{n, \lambda}=\left(2 n_{j}+1\right)|\lambda| H_{n, \lambda} \quad \text { and } \quad-\Delta_{\mathrm{osc}}^{\lambda} H_{n, \lambda}=(2|n|+d)|\lambda| H_{n, \lambda} .
$$

Our alternative definition of the Fourier transform on $\mathbb{H}^{d}$ then reads as follows:

Definition 1.2. - Let $\widetilde{\mathbb{H}}^{d} \stackrel{\text { def }}{=} \mathbb{N}^{2 d} \times \mathbb{R} \backslash\{0\}$, and denote by $\widehat{w}=(n, m, \lambda)$ a generic point of $\widetilde{\mathbb{H}}^{d}$. For $f$ in $L^{1}\left(\mathbb{H}^{d}\right)$, we define the map $\mathcal{F}_{\mathbb{H}} f$ (also denoted by $\widehat{f}_{\mathbb{H}}$ ) to be

$$
\mathcal{F}_{\mathbb{H}} f:\left\{\begin{array}{rll}
\widetilde{\mathbb{H}}^{d} & \longrightarrow \mathbb{C} \\
\widehat{w} & \longmapsto\left(\mathfrak{F}_{\mathbb{H}}(f)(\lambda) H_{m, \lambda} \mid H_{n, \lambda}\right)_{L^{2}} .
\end{array}\right.
$$

To underline the similarity between that definition and the classical one in $\mathbb{R}^{n}$, one may further compute $\left(\mathfrak{F}_{\mathbb{H}}(f)(\lambda) H_{m, \lambda} \mid H_{n, \lambda}\right)_{L^{2}}$. One can observe that, after a change of variable, the Fourier transform recasts in terms of the mean value of $f$ modulated by some oscillatory functions which are closely related to Wigner transforms of Hermite 
functions, namely

$$
\begin{gathered}
\mathcal{F}_{\mathbb{H}} f(\widehat{w})=\int_{\mathbb{H}^{d}} \overline{e^{i s \lambda} \mathcal{W}(\widehat{w}, Y)} f(Y, s) \mathrm{d} Y \mathrm{~d} s \\
\text { with } \quad \mathcal{W}(\widehat{w}, Y) \stackrel{\text { def }}{=} \int_{\mathbb{R}^{d}} e^{2 i \lambda\langle\eta, z\rangle} H_{n, \lambda}(y+z) H_{m, \lambda}(-y+z) \mathrm{d} z .
\end{gathered}
$$

With this new point of view, Formula (1.7) recasts as follows:

$$
\mathcal{F}_{\mathbb{H}}\left(\Delta_{\mathbb{H}} f\right)(\widehat{w})=-4|\lambda|(2|m|+d) \widehat{f}_{\mathbb{H}}(\widehat{w}) .
$$

Furthermore, if we endow the set $\widetilde{\mathbb{H}}^{d}$ with the measure $d \widehat{w}$ defined by the relation

$$
\int_{\widetilde{\mathbb{H}}^{d}} \theta(\widehat{w}) d \widehat{w} \stackrel{\text { def }}{=} \sum_{(n, m) \in \mathbb{N}^{2 d}} \int_{\mathbb{R}} \theta(n, m, \lambda)|\lambda|^{d} \mathrm{~d} \lambda,
$$

then the classical inversion formula and Fourier-Plancherel theorem become:

TheOREM 1.3. - Let $f$ be a function in $\mathcal{S}\left(\mathbb{H}^{d}\right)$. Then we have the inversion formula

$$
f(w)=\frac{2^{d-1}}{\pi^{d+1}} \int_{\widetilde{\mathbb{H}}^{d}} e^{i s \lambda} \mathcal{W}(\widehat{w}, Y) \widehat{f}_{\mathbb{H}}(\widehat{w}) \mathrm{d} \widehat{w} \quad \text { for any } w \text { in } \mathbb{H}^{d},
$$

and the Fourier transform $\mathcal{F}_{\mathbb{H}}$ can be extended to a bicontinuous isomorphism between the spaces $L^{2}\left(\mathbb{H}^{d}\right)$ and $L^{2}\left(\widetilde{\mathbb{H}}^{d}\right)$, that satisfies

$$
\left\|\widehat{f}_{\mathbb{H}_{H}}\right\|_{L^{2}\left(\widetilde{\mathbb{H}}^{d}\right)}^{2}=\frac{\pi^{d+1}}{2^{d-1}}\|f\|_{L^{2}\left(\mathbb{H}^{d}\right)}^{2} .
$$

Finally, for any couple $(f, g)$ of integrable functions, the following convolution identity holds true:

$$
\begin{aligned}
\mathcal{F}_{\mathbb{H}}(f \star g)(n, m, \lambda)= & \left(\widehat{f}_{\mathbb{H}} \cdot \widehat{g}_{\mathbb{H}}\right)(n, m, \lambda) \\
& \text { with }\left(\widehat{f}_{\mathbb{H}} \cdot \widehat{g}_{\mathbb{H}}\right)(n, m, \lambda) \stackrel{\text { def }}{=} \sum_{\ell \in \mathbb{N}^{d}} \widehat{f}_{\mathbb{H}}(n, \ell, \lambda) \widehat{g}_{\mathbb{H}}(\ell, m, \lambda) .
\end{aligned}
$$

For the reader's convenience, we shall present a proof of Theorem 1.3 in the appendix.

\section{Main results}

As already mentioned, our final goal is to extend the Fourier transform to tempered distributions on $\mathbb{H}^{d}$. If we follow the standard approach of the Euclidean setting that is described by (1.2) and (1.3), then the main difficulty is to get a description of the range $\mathcal{S}\left(\widetilde{\mathbb{H}}^{d}\right)$ of $\mathcal{S}\left(\mathbb{H}^{d}\right)$ by $\mathcal{F}_{\mathbb{H}}$ that allows to find out some bilinear form $\mathcal{B}_{\mathbb{H}}$ for identifying ${ }^{t} \mathcal{F}_{\mathbb{H}}$ with $\mathcal{F}_{\mathbb{H}}$.

Here, we first present results pertaining to the characterization of the set $\mathcal{S}\left(\widetilde{\mathbb{H}}^{d}\right)$, then exhibit a large class of functions of $\mathcal{S}\left(\widetilde{\mathbb{H}}^{d}\right)$ that, in particular, contains the Fourier transform of the heat kernel. Third, we go to examples of tempered distributions on the frequency set $\widetilde{\mathbb{H}}^{d}$ and, finally, to computations of Fourier transform of tempered distributions (with a special attention to functions independent of the vertical variable). 
To characterize $\mathcal{F}_{\mathbb{H}}\left(\mathcal{S}\left(\mathbb{H}^{d}\right)\right)$, we first have to keep in mind that, as in the Euclidean setting, we expect the Fourier transform to change the regularity of functions on $\mathbb{H}^{d}$ to decay of the Fourier transform. This is achieved in the following lemma that has been proved in $[\mathrm{BCD}]$.

Lemma 2.1. - For any integer $p$, there exist an integer $N_{p}$ and a positive constant $C_{p}$ such that for all $\widehat{w}$ in $\widetilde{\mathbb{H}}^{d}$ and all $f$ in $\mathcal{S}\left(\mathbb{H}^{d}\right)$, we have

$$
(1+|\lambda|(|n|+|m|+d)+|n-m|)^{p}\left|\widehat{f}_{\mathbb{H}}(n, m, \lambda)\right| \leqslant C_{p}\|f\|_{N_{p}, \mathcal{S}},
$$

where $\|\cdot\|_{N, \mathcal{S}}$ denotes the classical family of semi-norms of $\mathcal{S}\left(\mathbb{R}^{2 d+1}\right)$, namely

$$
\|f\|_{N, \mathcal{S}} \stackrel{\text { def }}{=} \sup _{|\alpha| \leqslant N}\left\|\left(1+|Y|^{2}+s^{2}\right)^{N / 2} \partial_{Y, s}^{\alpha} f\right\|_{L^{\infty}} .
$$

The second property we expect to have is that the Fourier transform changes decay into regularity. As in the Euclidean case, this is closely related to how it acts on weight functions: we expect $\mathcal{F}_{\mathbb{H}}$ to transform multiplication by a (suitable) weight function into a derivative operator on $\widetilde{\mathbb{H}}^{d}$. So far however, we lack a notion of differentiation on $\widetilde{\mathbb{H}}^{d}$ that could fit the scope. This is the aim of the following definition (see also Proposition A.3 in Appendix):

Definition 2.2. - For any function $\theta: \widetilde{\mathbb{H}}^{d} \rightarrow \mathbb{C}$ we define

$$
\begin{aligned}
\widehat{\Delta} \theta(\widehat{w}) \stackrel{\text { def }}{=}-\frac{1}{2|\lambda|}(\mid n & +m \mid+d) \theta(\widehat{w}) \\
& +\frac{1}{2|\lambda|} \sum_{j=1}^{d}\left(\sqrt{\left(n_{j}+1\right)\left(m_{j}+1\right)} \theta\left(\widehat{w}_{j}^{+}\right)+\sqrt{n_{j} m_{j}} \theta\left(\widehat{w}_{j}^{-}\right)\right)
\end{aligned}
$$

and, if in addition $\theta$ is differentiable with respect to $\lambda$,

$$
\begin{aligned}
\widehat{\mathcal{D}}_{\lambda} \theta(\widehat{w}) \stackrel{\text { def }}{=} \frac{\mathrm{d} \theta}{\mathrm{d} \lambda}(\widehat{w})+ & \frac{d}{2 \lambda} \theta(\widehat{w}) \\
& +\frac{1}{2 \lambda} \sum_{j=1}^{d}\left(\sqrt{n_{j} m_{j}} \theta\left(\widehat{w}_{j}^{-}\right)-\sqrt{\left(n_{j}+1\right)\left(m_{j}+1\right)} \theta\left(\widehat{w}_{j}^{+}\right)\right),
\end{aligned}
$$

where $\widehat{w}_{j}^{ \pm} \stackrel{\text { def }}{=}\left(n \pm \delta_{j}, m \pm \delta_{j}, \lambda\right)$ and $\delta_{j}$ denotes the element of $\mathbb{N}^{d}$ with all components equal to 0 except the $j$-th which has value 1 .

The notation in the above definition is justified by the following lemma that will be proved in Subsection 3.2. by

Lemma 2.3. - Let $M^{2}$ and $M_{0}$ be the multiplication operators defined on $\mathcal{S}\left(\mathbb{H}^{d}\right)$

$$
\left(M^{2} f\right)(Y, s) \stackrel{\text { def }}{=}|Y|^{2} f(Y, s) \text { and } \quad M_{0} f(Y, s) \stackrel{\text { def }}{=}-i s f(Y, s) .
$$

Then for all $f$ in $\mathcal{S}\left(\mathbb{H}^{d}\right)$, the following two relations hold true on $\widetilde{\mathbb{H}}^{d}$ :

$$
\mathcal{F}_{\mathbb{H}} M^{2} f=-\widehat{\Delta} \mathcal{F}_{\mathbb{H}} f \quad \text { and } \quad \mathcal{F}_{\mathbb{H}}\left(M_{0} f\right)=\widehat{\mathcal{D}}_{\lambda} \mathcal{F}_{\mathbb{H}} f
$$


The third aspect of regularity for functions in $\mathcal{F}_{\mathbb{H}}\left(\mathcal{S}\left(\mathbb{H}^{d}\right)\right)$ we have to underline is the link between their values for positive $\lambda$ and negative $\lambda$. This link is crucial in the computation of $M_{0} \mathcal{F}_{\mathbb{H}}^{-1}$ which is done in the proof of the inversion theorem in the Schwartz space $\mathcal{S}\left(\mathbb{H}^{d}\right)$. That property has no equivalent in the Euclidean setting, and is described in the following lemma:

Lemma 2.4. - Let us consider on $\mathcal{S}\left(\mathbb{H}^{d}\right)$ the operator $\mathcal{P}$ defined by

$$
\mathcal{P}(f)(Y, s) \stackrel{\text { def }}{=} \frac{1}{2} \int_{-\infty}^{s}\left(f\left(Y, s^{\prime}\right)-f\left(Y,-s^{\prime}\right)\right) \mathrm{d} s^{\prime} .
$$

Then, $\mathcal{P}$ maps continuously $\mathcal{S}\left(\mathbb{H}^{d}\right)$ to $\mathcal{S}\left(\mathbb{H}^{d}\right)$ and, for any $f$ in $\mathcal{S}\left(\mathbb{H}^{d}\right)$ and $\widehat{w}$ in $\widetilde{\mathbb{H}}^{d}$, we have $2 i \mathcal{F}_{\mathbb{H}}(\mathcal{P} f)=\widehat{\Sigma}_{0}\left(\mathcal{F}_{\mathbb{H}} f\right)$ with

$$
\widehat{\Sigma}_{0}(\theta)(\widehat{w}) \stackrel{\text { def }}{=} \frac{\theta(n, m, \lambda)-(-1)^{|n+m|} \theta(m, n,-\lambda)}{\lambda} .
$$

Technically, the above weird relation stems from the fact that the function $\mathcal{W}$ fulfills:

$$
\forall(n, m, \lambda, Y) \in \widetilde{\mathbb{H}}^{d} \times T^{\star} \mathbb{R}^{d}, \mathcal{W}(n, m, \lambda, Y)=(-1)^{|n+m|} \mathcal{W}(m, n,-\lambda, Y) .
$$

Note that, since the left-hand side of (2.6) belongs to the space $\mathcal{F}_{\mathbb{H}}\left(\mathcal{S}\left(\mathbb{H}^{d}\right)\right)$ that is the natural candidate for being $\mathcal{S}\left(\widetilde{\mathbb{H}}^{d}\right)$, we need to prescribe in addition to the decay properties pointed out in Lemmas 2.1 and 2.3 some condition involving $\widehat{\Sigma}_{0}$. This motivates the following definition.

DEFINITION 2.5. - We define $\mathcal{S}\left(\widetilde{\mathbb{H}}^{d}\right)$ to be the set of functions $\theta$ on $\widetilde{\mathbb{H}}^{d}$ such that:

- for any $(n, m)$ in $\mathbb{N}^{2 d}$, the map $\lambda \longmapsto \theta(n, m, \lambda)$ is smooth on $\mathbb{R} \backslash\{0\}$,

- for any non negative integer $N$, the functions $\widehat{\Delta}^{N} \theta, \widehat{\mathcal{D}}_{\lambda}^{N} \theta$ and $\widehat{\Sigma}_{0} \widehat{\mathcal{D}}_{\lambda}^{N} \theta$ decay faster than any power of $\widehat{d}_{0}(\widehat{w}) \stackrel{\text { def }}{=}|\lambda|(|n+m|+d)+|m-n|$.

We equip $\mathcal{S}\left(\widetilde{\mathbb{H}}^{d}\right)$ with the family of semi-norms

$$
\|\theta\|_{N, N^{\prime}, \mathcal{S}\left(\widetilde{\mathbb{H}}^{d}\right)} \stackrel{\text { def }}{=} \sup _{\widehat{w} \in \mathbb{\mathbb { H }}^{d}}\left(1+\widehat{d}_{0}(\widehat{w})\right)^{N}\left(\left|\widehat{\Delta}^{N^{\prime}} \theta(\widehat{w})\right|+\left|\widehat{\mathcal{D}}_{\lambda}^{N^{\prime}} \theta(\widehat{w})\right|+\left|\widehat{\Sigma}_{0} \widehat{\mathcal{D}}_{\lambda}^{N^{\prime}} \theta(\widehat{w})\right|\right) .
$$

Clearly, the above definition of semi-norms guarantees that there exist an integer $K$ and a positive real number $C$ so that the following inequality holds true:

$$
\|\theta\|_{L^{1}\left(\widetilde{\mathbb{H}}^{d}\right)} \leqslant C\|\theta\|_{K, 0, \mathcal{S}\left(\widetilde{\mathbb{H}}^{d}\right)} .
$$

More importantly, we have the following isomorphism theorem.

ThEOREM 2.6. - The Fourier transform $\mathcal{F}_{\mathbb{H}}$ is a bicontinuous isomorphism between $\mathcal{S}\left(\mathbb{H}^{d}\right)$ and $\mathcal{S}\left(\widetilde{\mathbb{H}}^{d}\right)$, and the inverse map is given by

$$
\widetilde{\mathcal{F}}_{\mathbb{H}} \theta(w) \stackrel{\text { def }}{=} \frac{2^{d-1}}{\pi^{d+1}} \int_{\widetilde{\mathbb{H}}^{d}} e^{i s \lambda} \mathcal{W}(\widehat{w}, Y) \theta(\widehat{w}) \mathrm{d} \widehat{w} .
$$

We shall discover throughout the paper that the definition of $\mathcal{S}\left(\widetilde{\mathbb{H}}^{d}\right)$ encodes a number of nontrivial hidden informations that are consequences of the sub-ellipticity of $\Delta_{\mathbb{H}}$. For instance, the stability of $\mathcal{S}\left(\widetilde{\mathbb{H}}^{d}\right)$ by the multiplication law defined in (1.13) 
is a consequence of the stability of $\mathcal{S}\left(\mathbb{H}^{d}\right)$ by convolution and of Theorem 2.6. Another hidden information is the behavior of functions of $\mathcal{S}\left(\widetilde{\mathbb{H}}^{d}\right)$ when $\lambda$ tends to 0 .

Keeping the decay inequality (2.1) in mind, we endow the set $\widetilde{\mathbb{H}}^{d}$ with the distance:

$$
\widehat{d}\left(\widehat{w}, \widehat{w}^{\prime}\right) \stackrel{\text { def }}{=}\left|\lambda(n+m)-\lambda^{\prime}\left(n^{\prime}+m^{\prime}\right)\right|_{1}+\left|(n-m)-\left(n^{\prime}-m^{\prime}\right)\right|+d\left|\lambda-\lambda^{\prime}\right|,
$$

where $|\cdot|_{1}$ denotes the Manhattan norm $\ell^{1}$ on $\mathbb{R}^{d}$.

With this choice, the Fourier transform of any function of $\mathcal{S}\left(\mathbb{H}^{d}\right)$ (and even of any integrable function on $\mathbb{H}^{d}$ ) is uniformly continuous on $\widetilde{\mathbb{H}}^{d}$, and can thus be extended by continuity on the completion $\widehat{\mathbb{H}}^{d}$ of $\widetilde{\mathbb{H}}^{d}$, as explained in greater details in the following statement that has been proved in [BCD]:

THEOREM 2.7. - The completion of $\left(\widetilde{\mathbb{H}}^{d}, \widehat{d}\right)$ is the metric space $\left(\widehat{\mathbb{H}}^{d}, \widehat{d}\right)$ where

$$
\widehat{\mathbb{H}}^{d} \stackrel{\text { def }}{=} \widetilde{\mathbb{H}}^{d} \cup \widehat{\mathbb{H}}_{0}^{d} \quad \text { with } \quad \widehat{\mathbb{H}}_{0}^{d} \stackrel{\text { def }}{=} \mathbb{R}_{\mp}^{d} \times \mathbb{Z}^{d} \quad \text { and } \quad \mathbb{R}_{\mp}^{d} \stackrel{\text { def }}{=}\left(\mathbb{R}_{-}\right)^{d} \cup\left(\mathbb{R}_{+}\right)^{d}
$$

and the extended distance (still denoted by $\widehat{d})$ is given for all $\widehat{w}=(n, m, \lambda)$ and $\widehat{w}^{\prime}=$ $\left(n^{\prime}, m^{\prime}, \lambda^{\prime}\right)$ in $\widetilde{\mathbb{H}}^{d}$, and for all $(\dot{x}, k)$ and $\left(\dot{x}^{\prime}, k^{\prime}\right)$ in $\widehat{\mathbb{H}}_{0}^{d}$ by

$$
\begin{aligned}
\widehat{d}\left(\widehat{w}, \widehat{w}^{\prime}\right) & =\left|\lambda(n+m)-\lambda^{\prime}\left(n^{\prime}+m^{\prime}\right)\right|_{1}+\left|(m-n)-\left(m^{\prime}-n^{\prime}\right)\right|+d\left|\lambda-\lambda^{\prime}\right|, \\
\widehat{d}(\widehat{w},(\dot{x}, k)) & =\widehat{d}((\dot{x}, k), \widehat{w}) \stackrel{\text { def }}{=}|\lambda(n+m)-\dot{x}|_{1}+|m-n-k|+d|\lambda|, \\
\widehat{d}\left((\dot{x}, k),\left(\dot{x}^{\prime}, k^{\prime}\right)\right) & =\left|\dot{x}-\dot{x}^{\prime}\right|_{1}+\left|k-k^{\prime}\right| .
\end{aligned}
$$

The Fourier transform $\widehat{f}_{\mathbb{H}}$ of any integrable function on $\mathbb{H}^{d}$ may be extended continuously to the whole set $\widehat{\mathbb{H}}^{d}$. Still denoting by $\widehat{f}_{\mathbb{H}}\left(\right.$ or $\mathcal{F}_{\mathbb{H}} f$ ) that extension, the linear map $\mathcal{F}_{\mathbb{H}}: f \mapsto \widehat{f}_{\mathbb{H}}$ is continuous from the space $L^{1}\left(\mathbb{H}^{d}\right)$ to the space $\mathcal{C}_{0}\left(\widehat{\mathbb{H}}^{d}\right)$ of continuous functions on $\widehat{\mathbb{H}}^{d}$ tending to 0 at infinity.

The above theorem prompts us to consider the space $\mathcal{S}\left(\widehat{\mathbb{H}}^{d}\right)$ of functions on $\widehat{\mathbb{H}}^{d}$ which are continuous extensions of elements of $\mathcal{S}\left(\widetilde{\mathbb{H}}^{d}\right)$, endowed with the semi-norms $\|\cdot\|_{N, N^{\prime}, \mathcal{S}\left(\widetilde{\mathbb{H}}^{d}\right)}$. Those semi-norms will be denoted by $\|\cdot\|_{N, N^{\prime}, \mathcal{S}\left(\widehat{\mathbb{H}}^{d}\right)}$ in all that follows.

Note that for any function $\theta$ in $\mathcal{S}\left(\widehat{\mathbb{H}}^{d}\right)$, having $\widehat{w}$ tend to $(\dot{x}, k)$ in $(2.6)$ yields

$$
\theta(\dot{x}, k)=(-1)^{|k|} \theta(-\dot{x},-k) .
$$

As regards convolution, we obtain, after passing to the limit in (1.13), the following noteworthy formula, valid for any two functions $f$ and $g$ in $L^{1}\left(\mathbb{H}^{d}\right)$ :

$$
\begin{aligned}
\mathcal{F}_{\mathbb{H}}(f \star g)_{\widehat{\mathbb{H}}_{0}^{d}}=\left(\mathcal{F}_{\mathbb{H}} f\right)_{\widehat{\mathbb{H}}_{0}^{d}} \stackrel{\widehat{\mathbb{H}}_{0}^{d}}{\stackrel{0}{(}}\left(\mathcal{F}_{\mathbb{H}} g\right)_{\widehat{\mathbb{H}}_{0}^{d}} \\
\text { with } \quad\left(\theta_{1} \stackrel{\widehat{\mathbb{H}}_{0}^{d}}{\cdot} \theta_{2}\right)(\dot{x}, k) \stackrel{\text { def }}{=} \sum_{k^{\prime} \in \mathbb{Z}^{d}} \theta_{1}\left(\dot{x}, k^{\prime}\right) \theta_{2}\left(\dot{x}, k-k^{\prime}\right) .
\end{aligned}
$$

Let us underline that the above product law (2.10) is commutative even though convolution of functions on the Heisenberg group is not (see (1.13)). 
The next question is how to extend the measure $\mathrm{d} \widehat{w}$ to $\widehat{\mathbb{H}}^{d}$. In fact, we have for any positive real numbers $R$ and $\varepsilon$,

$$
\begin{aligned}
\int_{\widehat{\mathbb{H}}^{d}} \mathbb{1}_{\{|\lambda||n+m|+|m-n| \leqslant R\}} \mathbb{1}_{|\lambda| \leqslant \varepsilon} \mathrm{d} \widehat{w} & =\int_{-\varepsilon}^{\varepsilon}\left(\sum_{n, m} \mathbb{1}_{\{|\lambda||n+m|+|m-n| \leqslant R\}}\right)|\lambda|^{d} \mathrm{~d} \lambda \\
& \leqslant C R^{2 d} \varepsilon .
\end{aligned}
$$

Therefore, it is natural to extend $\mathrm{d} \widehat{w}$ by 0 on $\widehat{\mathbb{H}}_{0}^{d}$. With this convention, keeping the same notation for the extended measure, we have for all continuous compactly supported function $\theta$ on $\widehat{\mathbb{H}}^{d}$,

$$
\int_{\widehat{\mathbb{H}}^{d}} \theta(\widehat{w}) \mathrm{d} \widehat{w} \stackrel{\text { def }}{=} \int_{\widetilde{\mathbb{H}}^{d}} \theta(\widehat{w}) \mathrm{d} \widehat{w} .
$$

At this stage of the paper, pointing out non-trivial examples of functions in $\mathcal{S}\left(\widehat{\mathbb{H}}^{d}\right)$ is highly informative. To this end, we introduce the set $\mathcal{S}_{d}^{+}$of smooth functions $f$ on $\left[0, \infty\left[^{d} \times \mathbb{Z}^{d} \times \mathbb{R}\right.\right.$ such that for any integer $p$, we have

$$
\sup _{\substack{\left(x_{1}, \ldots, x_{d}, k, \lambda\right) \in\left[0, \infty\left[{ }^{d} \times \mathbb{Z}^{d} \times \mathbb{R} \\|\alpha| \leqslant p\right.\right.}}\left(1+x_{1}+\ldots+x_{d}+|k|\right)^{p}\left|\partial_{x, \lambda}^{\alpha} f\left(x_{1}, \ldots, x_{d}, k, \lambda\right)\right|<\infty .
$$

As may be easily checked by the reader, the space $\mathcal{S}_{d}^{+}$is stable under derivation and multiplication by polynomial functions of $(x, k)$.

TheOrem 2.8. - Let $f$ be a function in $\mathcal{S}_{d}^{+}$. Let us define for $\widehat{w}=(n, m, \lambda)$ in $\widetilde{\mathbb{H}}^{d}$,

$$
\Theta_{f}(\widehat{w}) \stackrel{\text { def }}{=} f(|\lambda| R(n, m), m-n, \lambda) \quad \text { with } \quad R(n, m) \stackrel{\text { def }}{=}\left(n_{j}+m_{j}+1\right)_{1 \leqslant j \leqslant d} .
$$

If $f$ is supported in $\left[0, \infty\left[^{d} \times\{0\} \times \mathbb{R}\right.\right.$, or if $f$ is supported in $\left[r_{0}, \infty\left[^{d} \times \mathbb{Z}^{d} \times \mathbb{R}\right.\right.$ for some positive real number $r_{0}$, and satisfies

$$
f(x,-k,-\lambda)=(-1)^{|k|} f(x, k, \lambda) \quad \text { for all } k \in \mathbb{Z}^{d}, \lambda>0 \text { and } \quad x>0,
$$

then $\Theta_{f}$ belongs to $\mathcal{S}\left(\widehat{\mathbb{H}}^{d}\right)$.

A particularly striking consequence of Theorem 2.8 is that it provides a trivial proof of Hulanicki's theorem in [Hul84] and of the fact that the fundamental solution of the heat equation in $\mathbb{H}^{d}$ for the sublaplacian belongs to $\mathcal{S}\left(\mathbb{H}^{d}\right)$ (a nontrivial result that is usually deduced from the explicit formula established by B. Gaveau in [Gav77]).

Corollary 2.9. - For any function $a$ in $\mathcal{S}(\mathbb{R})$, there exists a function $h_{a}$ in $\mathcal{S}\left(\mathbb{H}^{d}\right)$ such that

$$
\forall f \in \mathcal{S}\left(\mathbb{H}^{d}\right), \quad a\left(-\Delta_{\mathbb{H}}\right) f=f \star h_{a} .
$$

In particular, the function $h$ such that, for all $u_{0}$ in $L^{1}\left(\mathbb{H}^{d}\right)$ and $t>0$, the map

$$
u: t \longmapsto u_{0} \star h_{t} \quad \text { with } h_{t}(y, \eta, s) \stackrel{\text { def }}{=} \frac{1}{t^{d+1}} h\left(\frac{y}{\sqrt{t}}, \frac{\eta}{\sqrt{t}}, \frac{s}{t}\right)
$$

fulfills

$$
\left.\partial_{t} u-\Delta_{\mathbb{H}} u=0 \text { in }\right] 0,+\infty\left[\times \mathbb{H}^{d} \text { and } \lim _{t \rightarrow 0^{+}} u(t)=u_{0} \text { in } L^{1}\left(\mathbb{H}^{d}\right),\right.
$$

belongs to $\mathcal{S}\left(\mathbb{H}^{d}\right)$. 
Proof. - Let us focus on the second result, proving the first one being similar. Then applying the Fourier transform with respect to the Heisenberg variable gives that $u$ must fulfill

$$
\widehat{u}_{\mathbb{H}}(t, n, m, \lambda)=e^{-4 t|\lambda|(2|m|+d)} \widehat{u}_{0}(n, m, \lambda) .
$$

At the same time, we have $u(t)=u_{0} \star h_{t}$. Hence, combining the convolution formula (1.13) and identity (2.12), we gather that

$$
\widehat{h}_{\mathbb{H}}(\widehat{w})=e^{-4|\lambda|(2|n|+d)} \mathbb{1}_{\{n=m\}} .
$$

Then applying Theorem 2.8 to the function $e^{-4\left(x_{1}+\ldots+x_{d}\right)} \mathbb{1}_{\{k=0\}}$ ensures that $\widehat{h}_{\mathbb{H}}$ belongs to $\mathcal{S}\left(\widehat{\mathbb{H}}^{d}\right)$, and the inversion theorem 2.6 eventually implies that $h$ is in $\mathcal{S}\left(\mathbb{H}^{d}\right)$.

Before explaining how to extend the Fourier transform to tempered distributions, let us specify what a tempered distribution on $\widehat{\mathbb{H}}^{d}$ is.

Definition 2.10. — Tempered distributions on $\widehat{\mathbb{H}}^{d}$ are elements of the set $\mathcal{S}^{\prime}\left(\widehat{\mathbb{H}}^{d}\right)$ of continuous linear forms on the Fréchet space $\mathcal{S}\left(\widehat{\mathbb{H}}^{d}\right)$.

Convergence of tempered distributions is defined in the standard way: we say that a sequence $\left(\mathcal{T}_{n}\right)_{n \in \mathbb{N}}$ of $\mathcal{S}^{\prime}\left(\widehat{\mathbb{H}}^{d}\right)$ converges to a tempered distribution $\mathcal{T}$ if

$$
\forall \theta \in \mathcal{S}\left(\widehat{\mathbb{H}}^{d}\right), \lim _{n \rightarrow \infty}\left\langle\mathcal{T}_{n}, \theta\right\rangle_{\mathcal{S}^{\prime}\left(\widehat{\mathbb{H}}^{d}\right) \times \mathcal{S}\left(\widehat{\mathbb{H}}^{d}\right)}=\langle\mathcal{T}, \theta\rangle_{\mathcal{S}^{\prime}\left(\widehat{\mathbb{H}}^{d}\right) \times \mathcal{S}\left(\widehat{\mathbb{H}}^{d}\right)} .
$$

A first class of examples of tempered distributions on $\widehat{\mathbb{H}}^{d}$ is given by "functions with moderate growth":

Definition 2.11. - We denote by $L_{M}^{1}\left(\widehat{\mathbb{H}}^{d}\right)$ the set of functions on $\widehat{\mathbb{H}}^{d}$ with moderate growth, that is the set of locally integrable functions $f$ on $\widehat{\mathbb{H}}^{d}$ such that there exists an integer $p$ satisfying

$$
\int_{\widehat{\mathbb{H}^{d}}}(1+|\lambda|(n+m \mid+d)+|n-m|)^{-p}|f(\widehat{w})| \mathrm{d} \widehat{w}<\infty .
$$

As in the Euclidean setting, functions in $L_{M}^{1}\left(\widehat{\mathbb{H}}^{d}\right)$ may be identified with tempered distributions:

TheOREM 2.12. - Let us consider $\iota$ be the map defined by

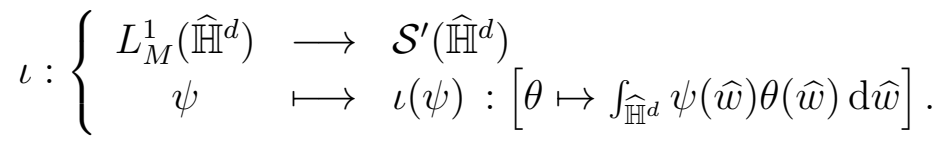

Then $\iota$ is a one-to-one linear map.

Moreover, if $p$ is an integer such that the map

$$
(n, m, \lambda) \longmapsto(1+|\lambda|(n+m \mid+d)+|n-m|)^{-p} f(n, m, \lambda)
$$

belongs to $L^{1}\left(\widehat{\mathbb{H}}^{d}\right)$, then we have

$$
|\langle\iota(f), \theta\rangle| \leqslant\left\|(1+|\lambda|(n+m \mid+d)+|n-m|)^{-p} f\right\|_{L^{1}\left(\widehat{\mathbb{H}}^{d}\right)}\|\theta\|_{p, 0, \mathcal{S}\left(\widehat{\mathbb{H}}^{d}\right)} .
$$

The following proposition that will be proved in Section 5 provides examples of functions in $L_{M}^{1}\left(\widehat{\mathbb{H}}^{d}\right)$. 
Proposition 2.13. - Let $\gamma<d+1$. Then the set $L_{M}^{1}\left(\widehat{\mathbb{H}}^{d}\right)$ contains the function $f_{\gamma}$ defined by

$$
f_{\gamma}(n, m, \lambda) \stackrel{\text { def }}{=}(|\lambda|(2|m|+d))^{-\gamma} \delta_{n, m}, \quad(n, m, \lambda) \in \widetilde{\mathbb{H}}^{d} .
$$

Remark 2.14. - The above proposition is no longer true for $\gamma=d+1$. If we look at the quantity $|\lambda|(2|n|+d)$ in $\widehat{\mathbb{H}}^{d}$ as an equivalent of $|\xi|^{2}$ for $\mathbb{R}^{d}$, then it means that the homogeneous dimension of $\widehat{\mathbb{H}}^{d}$ is $2 d+2$, as for $\mathbb{H}^{d}$.

Obviously, any Dirac mass on $\widehat{\mathbb{H}}^{d}$ is a tempered distribution. Let us also note that because

the linear form

$$
|\theta(n, n, \lambda)| \leqslant(1+|\lambda|(2|n|+d))^{-d-2}\|\theta\|_{d+2,0, \mathcal{S}\left(\widehat{\mathbb{H}}^{d}\right)},
$$

$$
\mathcal{I}:\left\{\begin{array}{cll}
\mathcal{S}\left(\widehat{\mathbb{H}}^{d}\right) & \longrightarrow \mathbb{C} \\
\theta & \longmapsto & \sum_{n \in \mathbb{N}^{d}} \int_{\mathbb{R}} \theta(n, n, \lambda)|\lambda|^{d} \mathrm{~d} \lambda
\end{array}\right.
$$

is a tempered distribution on $\widehat{\mathbb{H}}^{d}$.

We now want to exhibit tempered distributions on $\widehat{\mathbb{H}}^{d}$ which are not functions or measures. The following proposition states that the analogue on $\widehat{\mathbb{H}}^{d}$ of finite part distributions on $\mathbb{R}^{n}$, are indeed in $\mathcal{S}^{\prime}\left(\widehat{\mathbb{H}}^{d}\right)$.

Proposition 2.15. - Let $\gamma$ be in the interval $] d+1, d+3 / 2[$ and denote by $\hat{0}$ the element $(0,0)$ of $\widehat{\mathbb{H}}_{0}^{d}$. Then for any function $\theta$ in $\mathcal{S}\left(\widehat{\mathbb{H}}^{d}\right)$, the function defined a.e. on $\widehat{\mathbb{H}}^{d}$ by

$$
(n, m, \lambda) \longmapsto \delta_{n, m}\left(\frac{\theta(n, n, \lambda)+\theta(n, n,-\lambda)-2 \theta(\widehat{0})}{|\lambda|^{\gamma}(2|n|+d)^{\gamma}}\right),
$$

is integrable. Furthermore, the linear form defined by

$$
\left\langle\operatorname{Pf}\left(\frac{1}{|\lambda|^{\gamma}(2|n|+d)^{\gamma}}\right), \theta\right\rangle \stackrel{\text { def }}{=} \frac{1}{2} \int_{\widehat{\mathbb{H}}^{d}}\left(\frac{\theta(n, n, \lambda)+\theta(n, n,-\lambda)-2 \theta(\widehat{0})}{|\lambda|^{\gamma}(2|n|+d)^{\gamma}}\right) \delta_{n, m} \mathrm{~d} \widehat{w}
$$

is in $\mathcal{S}^{\prime}\left(\widehat{\mathbb{H}}^{d}\right)$, and its restriction to $\widetilde{\mathbb{H}}^{d}$ is the function $f_{\gamma}$ that has been defined above, that is, for any $\theta$ in $\mathcal{S}\left(\widehat{\mathbb{H}}^{d}\right)$ such that $\theta(n, n, \lambda)=0$ for small enough $|\lambda|(2|n|+d)$, we have

$$
\left\langle\operatorname{Pf}\left(\frac{1}{|\lambda|^{\gamma}(2|n|+d)^{\gamma}}\right), \theta\right\rangle=\int_{\widehat{\mathbb{H}}^{d}} f_{\gamma}(\widehat{w}) \theta(\widehat{w}) \mathrm{d} \widehat{w} .
$$

Another interesting example of tempered distribution on $\widehat{\mathbb{H}}^{d}$ is the measure $\mu_{\widehat{\mathbb{H}}_{0}^{d}}$ defined in Lemma 3.1 of [BCD]. In our setting, that result recasts as follows: by

Proposition 2.16. - Let the measure $\mu_{\widehat{\mathbb{H}}_{0}^{d}}$ be defined for all function $\theta$ in $\mathcal{S}\left(\widehat{\mathbb{H}}^{d}\right)$,

$$
\left\langle\mu_{\widehat{\mathbb{H}}_{0}^{d}}, \theta\right\rangle=\int_{\widehat{\mathbb{H}}_{0}^{d}} \theta(\dot{x}, k) \mathrm{d} \mu_{\widehat{\mathbb{H}}_{0}^{d}}(\dot{x}, k) \stackrel{\text { def }}{=} 2^{-d} \sum_{k \in \mathbb{Z}^{d}}\left(\int_{\left(\mathbb{R}_{-}\right)^{d}} \theta(\dot{x}, k) \mathrm{d} \dot{x}+\int_{\left(\mathbb{R}_{+}\right)^{d}} \theta(\dot{x}, k) \mathrm{d} \dot{x}\right) .
$$

Then $\mu_{\widehat{\mathbb{H}}_{0}^{d}}$ belongs to $\mathcal{S}^{\prime}\left(\widehat{\mathbb{H}}^{d}\right)$, and for any function $\psi$ in $\mathcal{S}(\mathbb{R})$ with integral 1 we have

$$
\lim _{\varepsilon \rightarrow 0} \frac{1}{\varepsilon} \psi\left(\frac{\lambda}{\varepsilon}\right)=\mu_{\widehat{\mathbb{H}}_{0}^{d}} \quad \text { in } \mathcal{S}^{\prime}\left(\widehat{\mathbb{H}}^{d}\right) .
$$


Let us finally explain how Formulae (1.2) and (1.3) may be adapted so as to extend the Fourier transform on $\mathbb{H}^{d}$ to tempered distributions. We introduce:

$$
\begin{aligned}
& \mathcal{B}_{\mathbb{H}}:\left\{\begin{array}{cll}
\mathcal{S}\left(\mathbb{H}^{d}\right) \times \mathcal{S}\left(\widehat{\mathbb{H}}^{d}\right) & \longrightarrow & \mathbb{C} \\
(f, \theta) & \longmapsto & \int_{\mathbb{H}^{d} \times \widehat{\mathbb{H}}^{d}} f(Y, s) \overline{e^{i s \lambda} \mathcal{W}(\widehat{w}, Y)} \theta(\widehat{w}) \mathrm{d} w \mathrm{~d} \widehat{w}
\end{array}\right.
\end{aligned}
$$

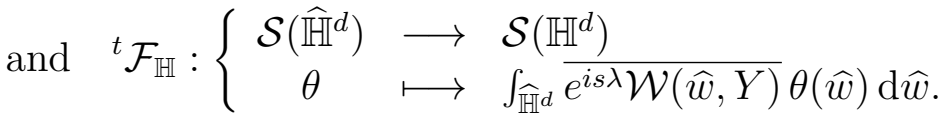

Since, for any $\theta$ in $\mathcal{S}\left(\widehat{\mathbb{H}}^{d}\right)$ and $w=(y, \eta, s)$ in $\mathbb{H}^{d}$, we have

$$
\left({ }^{t} \mathcal{F}_{\mathbb{H}} \theta\right)(y, \eta, s)=\frac{\pi^{d+1}}{2^{d-1}}\left(\mathcal{F}_{\mathbb{H}}^{-1} \theta\right)(y,-\eta,-s) .
$$

Theorem 2.6 ensures that ${ }^{t} \mathcal{F}_{\mathbb{H}}$ is a continuous isomorphism between $\mathcal{S}\left(\widehat{\mathbb{H}}^{d}\right)$ and $\mathcal{S}\left(\mathbb{H}^{d}\right)$. Now, we observe that for any $f$ in $\mathcal{S}\left(\mathbb{H}^{d}\right)$ and $\theta$ in $\mathcal{S}\left(\widehat{\mathbb{H}}^{d}\right)$, we have

$$
\mathcal{B}_{\mathbb{H}}(f, \theta)=\int_{\mathbb{H}^{d}} f(w)\left({ }^{t} \mathcal{F}_{\mathbb{H}} \theta\right)(w) \mathrm{d} w=\int_{\widehat{\mathbb{H}^{d}}}\left(\mathcal{F}_{\mathbb{H}} f\right)(\widehat{w}) \theta(\widehat{w}) \mathrm{d} \widehat{w} .
$$

This prompts us to extend $\mathcal{F}_{\mathbb{H}}$ on $\mathcal{S}^{\prime}\left(\mathbb{H}^{d}\right)$ by

$$
\left\langle\mathcal{F}_{\mathbb{H}} T, \theta\right\rangle_{\mathcal{S}^{\prime}\left(\widehat{\mathbb{H}}^{d}\right) \times \mathcal{S}\left(\widehat{\mathbb{H}}^{d}\right)} \stackrel{\text { def }}{=}\left\langle T,{ }^{t} \mathcal{F}_{\mathbb{H}} \theta\right\rangle_{\mathcal{S}^{\prime}\left(\mathbb{H}^{d}\right) \times \mathcal{S}\left(\mathbb{H}^{d}\right)} \quad \text { for all } \quad \theta \in \mathcal{S}\left(\widehat{\mathbb{H}}^{d}\right) .
$$

A direct consequence of this definition is the following statement:

Proposition 2.17. - The map $\mathcal{F}_{\mathbb{H}}$ defined just above is continuous and one-toone from $\mathcal{S}^{\prime}\left(\mathbb{H}^{d}\right)$ onto $\mathcal{S}^{\prime}\left(\widehat{\mathbb{H}}^{d}\right)$. Furthermore, its restriction to $L^{1}\left(\mathbb{H}^{d}\right)$ coincides with Definition 1.2.

Just to compare with the Euclidean case, let us give some examples of simple computations of Fourier transform of tempered distributions on $\mathbb{H}^{d}$.

Proposition 2.18. - We have

$$
\mathcal{F}_{\mathbb{H}}\left(\delta_{0}\right)=\mathcal{I} \quad \text { and } \quad \mathcal{F}_{\mathbb{H}}(\mathbb{1})=\frac{\pi^{d+1}}{2^{d-1}} \delta_{\widehat{0}}
$$

where $\mathcal{I}$ is defined by (2.14) and $\widehat{0}$ is the element of $\widehat{\mathbb{H}}_{0}^{d}$ corresponding to $\dot{x}=0$ and $k=0$.

Let us emphasize, that without completing the frequency set $\widetilde{\mathbb{H}}^{d}$, it would not be even possible to state the second result of the above proposition. The same happens in the following statement when computing the Fourier transform of a function independent of the vertical variable.

THEOREM 2.19. - We have for any integrable function $g$ on $T^{\star} \mathbb{R}^{d}$,

$$
\mathcal{F}_{\mathbb{H}}(g \otimes \mathbf{1})=\left(\mathcal{G}_{\mathbb{H}} g\right) \mu_{\widehat{\mathbb{H}}_{0}^{d}}
$$


where $\mathcal{G}_{\mathbb{H}} g$ is defined by

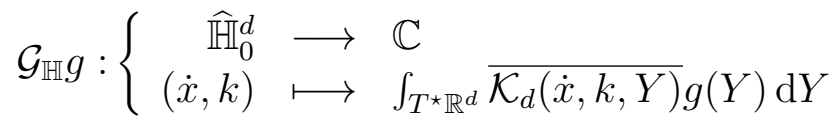

$$
\begin{aligned}
& \text { with } \mathcal{K}_{d}(\dot{x}, k, Y)=\bigotimes_{j=1}^{d} \mathcal{K}\left(\dot{x}_{j}, k_{j}, Y_{j}\right) \\
& \text { and } \mathcal{K}(\dot{x}, k, y, \eta) \stackrel{\text { def }}{=} \frac{1}{2 \pi} \int_{-\pi}^{\pi} e^{i\left(2 \mid \dot{x}^{\frac{1}{2}}(y \sin z+\eta \operatorname{sgn}(\dot{x}) \cos z)+k z\right)} \mathrm{d} z \text {. }
\end{aligned}
$$

The rest of the paper unfolds as follows. In Section 3, we prove Lemmas 2.3 and 2.4, and then Theorem 2.6. In Section 4, we establish Theorem 2.8. In Section 5, we study in full details the examples of tempered distributions on $\widehat{\mathbb{H}}^{d}$ given in Propositions 2.132.15, and Theorem 2.12. In Section 6, we prove Proposition 2.18 and Theorem 2.19. Further remarks as well as proofs within our setting of known results are postponed in the appendix.

\section{The range of the Schwartz class by the Fourier transform}

The present sectionaims at giving a handy characterization of the range of $\mathcal{S}\left(\mathbb{H}^{d}\right)$ by the Fourier transform. Our Ariadne thread throughout will be that we expect that, for the action of $\mathcal{F}_{\mathbb{H}}$, regularity implies decay and decay implies regularity. The answer to the first issue has been given in Lemma 2.1 (proved in [BCD]). Here we shall concentrate on the second issue, in connection with the definition of differentiation for functions on $\widetilde{\mathbb{H}}^{d}$, given in (2.2) and (2.3). To complete our analysis of the space $\mathcal{F}_{\mathbb{H}}\left(\mathcal{S}\left(\mathbb{H}^{d}\right)\right.$ ), we will have to get some information on the behavior of elements of $\mathcal{F}_{\mathbb{H}}\left(\mathcal{S}\left(\mathbb{H}^{d}\right)\right.$ ) for $\lambda$ going to 0 (that is in the neighborhood of the set $\widehat{\mathbb{H}}_{0}^{d}$ ). This is Lemma 2.4 that points out an extra and fundamental relationship between positive and negative $\lambda$ 's for functions of $\mathcal{F}_{\mathbb{H}}\left(\mathcal{S}\left(\mathbb{H}^{d}\right)\right)$.

A great deal of our program will be achieved by describing the action of the weight function $M^{2}$ and of the differentiation operator $\partial_{\lambda}$ on $\mathcal{W}$. This is the goal of the next paragraph.

\subsection{Some properties for Wigner transform of Hermite functions}

The following lemma describes the action of the weight function $M^{2}$ on $\mathcal{W}$.

Lemma 3.1. - For all $\widehat{w}$ in $\widetilde{\mathbb{H}}^{d}$ and $Y$ in $T^{\star} \mathbb{R}^{d}$, we have

$$
|Y|^{2} \mathcal{W}(\widehat{w}, Y)=-\widehat{\Delta} \mathcal{W}(\cdot, Y)(\widehat{w})
$$

where Operator $\widehat{\Delta}$ has been defined in (2.2). 
Proof. - From the definition of $\mathcal{W}$ and integrations by parts, we get

$$
\begin{aligned}
& |Y|^{2} \mathcal{W}(\widehat{w}, Y)=\int_{\mathbb{R}^{d}}\left(|y|^{2}-\frac{1}{4 \lambda^{2}} \Delta_{z}\right)\left(e^{2 i \lambda\langle\eta, z\rangle}\right) H_{n, \lambda}(y+z) H_{m, \lambda}(-y+z) \mathrm{d} z \\
& =\int_{\mathbb{R}^{d}} e^{2 i \lambda\langle\eta, z\rangle}|\lambda|^{\frac{d}{2}} \mathcal{I}(\widehat{w}, y, z) \mathrm{d} z \\
& \text { with } \mathcal{I}(\widehat{w}, y, z) \stackrel{\text { def }}{=}\left(|y|^{2}-\frac{1}{4 \lambda^{2}} \Delta_{z}\right)\left(H_{n}\left(|\lambda|^{\frac{1}{2}}(y+z)\right) H_{m}\left(|\lambda|^{\frac{1}{2}}(-y+z)\right)\right) \text {. }
\end{aligned}
$$

From Leibniz's rule, the chain rule and the following identity:

$$
4|y|^{2}=|y+z|^{2}+|y-z|^{2}+2(y+z) \cdot(y-z),
$$

we get

$$
\begin{aligned}
\mathcal{I}(\widehat{w}, y, z)=- & \frac{1}{4 \lambda^{2}}\left(\left(\Delta_{z}-\lambda^{2}|y+z|^{2}\right) H_{n}\left(|\lambda|^{\frac{1}{2}}(y+z)\right)\right) H_{m}\left(|\lambda|^{\frac{1}{2}}(-y+z)\right) \\
& -\frac{1}{4 \lambda^{2}}\left(\left(\Delta_{z}-\lambda^{2}|y-z|^{2}\right) H_{m}\left(|\lambda|^{\frac{1}{2}}(-y+z)\right)\right) H_{n}\left(|\lambda|^{\frac{1}{2}}(y+z)\right) \\
& -\frac{1}{2|\lambda|} \sum_{j=1}^{d}\left(\partial_{j} H_{n}\right)\left(|\lambda|^{\frac{1}{2}}(y+z)\right)\left(\partial_{j} H_{m}\right)\left(|\lambda|^{\frac{1}{2}}(-y+z)\right) \\
& -\frac{1}{2}(z+y) \cdot(z-y) H_{n}\left(|\lambda|^{\frac{1}{2}}(y+z)\right) H_{m}\left(|\lambda|^{\frac{1}{2}}(-y+z)\right) .
\end{aligned}
$$

Using (1.8), we end up with

$$
\begin{aligned}
\mathcal{I}(\widehat{w}, y, z)= & \frac{1}{2|\lambda|}(|n+m|+d) H_{n}\left(|\lambda|^{\frac{1}{2}}(y+z)\right) H_{m}\left(|\lambda|^{\frac{1}{2}}(-y+z)\right) \\
& -\frac{1}{2|\lambda|} \sum_{j=1}^{d}\left\{\left(\partial_{j} H_{n}\right)\left(|\lambda|^{\frac{1}{2}}(y+z)\right)\left(\partial_{j} H_{m}\right)\left(|\lambda|^{\frac{1}{2}}(-y+z)\right)\right. \\
& \left.+\left(M_{j} H_{n}\right)\left(|\lambda|^{\frac{1}{2}}(y+z)\right)\left(M_{j} H_{m}\right)\left(|\lambda|^{\frac{1}{2}}(-y+z)\right)\right\} .
\end{aligned}
$$

Then, taking advantage of (A.4), we get Identity (2.2).

The purpose of the following lemma is to investigate the action of $\partial_{\lambda}$ on $\mathcal{W}$.

Lemma 3.2. - We have, for all $\widehat{w}$ in $\widetilde{\mathbb{H}}^{d}$ and $Y \in T^{\star} \mathbb{R}^{d}$, the following formula:

$$
\partial_{\lambda} \mathcal{W}(\widehat{w}, Y)=\underline{\mathcal{D}}_{\lambda} \mathcal{W}(\widehat{w}, Y)
$$

where, for $\theta: \widetilde{\mathbb{H}}^{d} \rightarrow \mathbb{C}$, we denoted

$$
\widehat{\mathcal{D}}_{\lambda} \theta(\widehat{w}) \stackrel{\text { def }}{=}-\frac{d}{2 \lambda} \theta(\widehat{w})+\frac{1}{2 \lambda} \sum_{j=1}^{d}\left\{\sqrt{\left(n_{j}+1\right)\left(m_{j}+1\right)} \theta\left(\widehat{w}_{j}^{+}\right)-\sqrt{n_{j} m_{j}} \theta\left(\widehat{w}_{j}^{-}\right)\right\} .
$$


Proof. - Let us write that

$$
\begin{aligned}
\partial_{\lambda} \mathcal{W}(\widehat{w}, Y) & =\int_{\mathbb{R}^{d}} \frac{\mathrm{d}}{\mathrm{d} \lambda}\left(|\lambda|^{\frac{d}{2}} e^{2 i \lambda\langle\eta, z\rangle} H_{n}\left(|\lambda|^{\frac{1}{2}}(y+z)\right) H_{m}\left(|\lambda|^{\frac{1}{2}}(-y+z)\right)\right) \mathrm{d} z \\
& =\frac{d}{2 \lambda} \mathcal{W}(\widehat{w}, Y)+\mathcal{W}_{1}(\widehat{w}, Y)+\mathcal{W}_{2}(\widehat{w}, Y)
\end{aligned}
$$

with $\quad \mathcal{W}_{1}(\widehat{w}, Y) \stackrel{\text { def }}{=} \int_{\mathbb{R}^{d}} 2 i\langle\eta, z\rangle e^{2 i \lambda\langle\eta, z\rangle}|\lambda|^{\frac{d}{2}} H_{n}\left(|\lambda|^{\frac{1}{2}}(y+z)\right) H_{m}\left(|\lambda|^{\frac{1}{2}}(-y+z)\right) \mathrm{d} z$

and $\quad \mathcal{W}_{2}(\widehat{w}, Y) \stackrel{\text { def }}{=} \int_{\mathbb{R}^{d}} e^{2 i \lambda\langle\eta, z\rangle}|\lambda|^{\frac{d}{2}} \frac{\mathrm{d}}{\mathrm{d} \lambda}\left(H_{n}\left(|\lambda|^{\frac{1}{2}}(y+z)\right) H_{m}\left(|\lambda|^{\frac{1}{2}}(-y+z)\right)\right) \mathrm{d} z$.

As we have

$$
2 i\langle\eta, z\rangle e^{2 i \lambda\langle\eta, z\rangle}=\frac{1}{\lambda} \sum_{j=1}^{d} z_{j} \partial_{z_{j}} e^{2 i \lambda\langle\eta, z\rangle},
$$

an integration by parts gives

$$
\begin{aligned}
\mathcal{W}_{1}(\widehat{w}, Y)= & -\frac{d}{\lambda} \mathcal{W}(\widehat{w}, Y) \\
& -\frac{1}{\lambda} \sum_{j=1}^{d} \int_{\mathbb{R}^{d}} e^{2 i \lambda\langle\eta, z\rangle}|\lambda|^{\frac{d}{2}} z_{j} \partial_{z_{j}}\left(H_{n}\left(|\lambda|^{\frac{1}{2}}(y+z)\right) H_{m}\left(|\lambda|^{\frac{1}{2}}(-y+z)\right)\right) \mathrm{d} z .
\end{aligned}
$$

Now, let us compute

$$
\mathcal{J}(\widehat{w}, y, z) \stackrel{\text { def }}{=}\left(\frac{\mathrm{d}}{\mathrm{d} \lambda}-\frac{1}{\lambda} \sum_{j=1}^{d} z_{j} \partial_{z_{j}}\right)\left(H_{n}\left(|\lambda|^{\frac{1}{2}}(y+z)\right) H_{m}\left(|\lambda|^{\frac{1}{2}}(-y+z)\right)\right) .
$$

From the chain rule we get

$$
\begin{array}{r}
\mathcal{J}(\widehat{w}, y, z)=\frac{|\lambda|^{\frac{1}{2}}}{2 \lambda} \sum_{j=1}^{d}\left\{\left(y_{j}+z_{j}\right) H_{m}\left(|\lambda|^{\frac{1}{2}}(-y+z)\right)\left(\partial_{j} H_{n}\right)\left(|\lambda|^{\frac{1}{2}}(y+z)\right)\right. \\
+\left(-y_{j}+z_{j}\right) H_{n}\left(|\lambda|^{\frac{1}{2}}(y+z)\right)\left(\partial_{j} H_{m}\right)\left(|\lambda|^{\frac{1}{2}}(-y+z)\right) \\
-2 z_{j} H_{m}\left(|\lambda|^{\frac{1}{2}}(-y+z)\right)\left(\partial_{j} H_{n}\right)\left(|\lambda|^{\frac{1}{2}}(y+z)\right) \\
\left.-2 z_{j} H_{n}\left(|\lambda|^{\frac{1}{2}}(y+z)\right)\left(\partial_{j} H_{m}\right)\left(|\lambda|^{\frac{1}{2}}(-y+z)\right)\right\} .
\end{array}
$$

This gives

$$
\begin{array}{r}
\mathcal{J}(\widehat{w}, y, z)=-\frac{1}{2 \lambda} \sum_{j=1}^{d}\left\{\left(\partial_{j} H_{n}\right)\left(|\lambda|^{\frac{1}{2}}(y+z)\right)|\lambda|^{\frac{1}{2}}\left(-y_{j}+z_{j}\right) H_{m}\left(|\lambda|^{\frac{1}{2}}(-y+z)\right)\right. \\
\left.+|\lambda|^{\frac{1}{2}}\left(y_{j}+z_{j}\right) H_{n}\left(|\lambda|^{\frac{1}{2}}(y+z)\right)\left(\partial_{j} H_{m}\right)\left(|\lambda|^{\frac{1}{2}}(-y+z)\right)\right\},
\end{array}
$$

which writes

$$
\begin{aligned}
\mathcal{J}(\widehat{w}, y, z)=-\frac{1}{2 \lambda} \sum_{j=1}^{d}\left\{\left(\partial_{j} H_{n}\right)(\mid\right. & \left.\left.\lambda\right|^{\frac{1}{2}}(y+z)\right)\left(M_{j} H_{m}\right)\left(|\lambda|^{\frac{1}{2}}(-y+z)\right) \\
& \left.+\left(M_{j} H_{n}\right)\left(|\lambda|^{\frac{1}{2}}(y+z)\right)\left(\partial_{j} H_{m}\right)\left(|\lambda|^{\frac{1}{2}}(-y+z)\right)\right\} .
\end{aligned}
$$

Using Relations (A.4) completes the proof of the Lemma. 


\subsection{Decay provides regularity}

Granted with Lemmas 3.1 and 3.2, it is now easy to establish Lemma 2.3. Indeed, according to (1.9), we have

$$
\left(\mathcal{F}_{\mathbb{H}} M^{2} f\right)(\widehat{w})=\int_{\mathbb{H}^{d}} e^{-i s \lambda} f(Y, s)|Y|^{2} \overline{\mathcal{W}}(\widehat{w}, Y) \mathrm{d} Y \mathrm{~d} s .
$$

Therefore, Lemma 3.1 implies that

$$
\begin{aligned}
\left(\mathcal{F}_{\mathbb{H}} M^{2} f\right)(\widehat{w})= & \frac{1}{2|\lambda|}(|n+m|+d) \int_{\mathbb{H}^{d}} f(Y, s) e^{-i s \lambda} \overline{\mathcal{W}}(\widehat{w}, Y) \mathrm{d} Y \mathrm{~d} s \\
& -\frac{1}{2|\lambda|} \sum_{j=1}^{d}\left\{\sqrt{\left(n_{j}+1\right)\left(m_{j}+1\right)} \int_{\mathbb{H}^{d}} f(Y, s) e^{-i s \lambda} \overline{\mathcal{W}}\left(\widehat{w}_{j}^{+}, Y\right) \mathrm{d} Y \mathrm{~d} s\right. \\
& \left.+\sqrt{n_{j} m_{j}} \int_{\mathbb{H}^{d}} f(Y, s) e^{-i s \lambda} \overline{\mathcal{W}}\left(\widehat{w}_{j}^{-}, Y\right) \mathrm{d} Y \mathrm{~d} s\right\} .
\end{aligned}
$$

By the definition of the Fourier transform and of $\widehat{\Delta}$, this gives $\mathcal{F}_{\mathbb{H}} M^{2} f=-\widehat{\Delta} \mathcal{F}_{\mathbb{H}} f$.

To establish the second part of the lemma, we start from (1.9) and get

$$
\begin{aligned}
\mathcal{F}_{\mathbb{H}}\left(M_{0} f\right)(\widehat{w}) & =\int_{\mathbb{H}^{d}} \frac{\mathrm{d}}{\mathrm{d} \lambda}\left(e^{-i s \lambda}\right) f(Y, s) \overline{\mathcal{W}}(\widehat{w}, Y) \mathrm{d} Y \mathrm{~d} s \\
& =\frac{\mathrm{d}}{\mathrm{d} \lambda}\left(\mathcal{F}_{\mathbb{H}} f\right)(\widehat{w})-\int_{\mathbb{H}^{d}} e^{-i s \lambda} f(Y, s) \frac{\mathrm{d}}{\mathrm{d} \lambda}(\overline{\mathcal{W}}(\widehat{w}, Y)) \mathrm{d} Y \mathrm{~d} s .
\end{aligned}
$$

Rewriting the last term according to Formula (3.1), we discover that

$$
\left(\mathcal{F}_{\mathbb{H}} M_{0} f\right)(\widehat{w})=\frac{\mathrm{d}}{\mathrm{d} \lambda}\left(\mathcal{F}_{\mathbb{H}} f\right)(\widehat{w})-\underline{\mathcal{D}}_{\lambda} \mathcal{F}_{\mathbb{H}} f(\widehat{w}) .
$$

By the definition of the Fourier transform, this concludes the proof of Lemma 2.3.

On the one hand, Lemmas 2.1 and 2.3 guarantee that decay in the physical space provides regularity in the Fourier space, and that regularity gives decay. On the other hand, the relations we established so far do not give much insight on the behavior of the Fourier transform near $\widehat{\mathbb{H}}_{0}^{d}$ even though we know from Theorem 2.7 that in the case of an integrable function, it has to be uniformly continuous up to $\lambda=0$. Getting more information on the behavior of the Fourier transform of functions in $\mathcal{S}\left(\mathbb{H}^{d}\right)$ in a neighborhood of $\widehat{\mathbb{H}}_{0}^{d}$ is what we want to do now with the proof of Lemma 2.4.

Proof of Lemma 2.4. - Since the partial Fourier transform $\mathcal{F}_{s}$ with respect to the vertical variable is a bounded isomorphism on $\mathcal{S}\left(\mathbb{R}^{2 d+1}\right)$, it suffices to establish that $\mathcal{F}_{s} \mathcal{P}$ is a bounded operator on $\mathcal{S}\left(\mathbb{R}^{2 d+1}\right)$. Now, fix some function $f$ in $\mathcal{S}\left(\mathbb{H}^{d}\right)$, and observe that

$$
\partial_{s} \mathcal{P} f(Y, s)=\frac{1}{2}(f(Y, s)-f(Y,-s)) .
$$

Taking the Fourier transform with respect to the variable $s$ gives

$$
i \lambda \mathcal{F}_{s}(\mathcal{P} f)(Y, \lambda)=\frac{1}{2}\left(\mathcal{F}_{s} f(Y, \lambda)-\mathcal{F}_{s} f(Y,-\lambda)\right) .
$$


Let us consider a function $\chi$ in $\mathcal{S}(\mathbb{R})$ with value 1 near 0 and let us write

$$
\begin{aligned}
& i \mathcal{F}_{s}(\mathcal{P} f)(Y, \lambda) \\
& \quad=\frac{1-\chi(\lambda)}{2 \lambda}\left(\mathcal{F}_{s} f(Y, \lambda)-\mathcal{F}_{s} f(Y,-\lambda)\right)+\chi(\lambda) \int_{0}^{1}\left(\partial_{\lambda} \mathcal{F}_{s} f\right)(Y,-\lambda+2 t \lambda) \mathrm{d} t .
\end{aligned}
$$

The two terms on the right-hand side define bounded operators on $\mathcal{S}\left(\mathbb{R}^{2 d+1}\right)$. Hence, the operator $\mathcal{F}_{s} \mathcal{P}$ is bounded on $\mathcal{S}\left(\mathbb{R}^{2 d+1}\right)$ and thus, so does $\mathcal{P}$.

Note that in the case of a function $g$ in $\mathcal{S}\left(\mathbb{H}^{d}\right)$, Formula (1.9) may be alternately written:

$$
\mathcal{F}_{\mathbb{H}} g(\widehat{w})=\int_{T^{\star} \mathbb{R}^{d}} \mathcal{F}_{s} g(Y, \lambda) \overline{\mathcal{W}}(\widehat{w}, Y) \mathrm{d} Y \text { for all } \widehat{w}=(n, m, \lambda) \text { in } \widetilde{\mathbb{H}}^{d}
$$

Relations (2.7) and (3.2) guarantee that

$$
\begin{aligned}
2 i \lambda \mathcal{F}_{\mathbb{H}}(\mathcal{P} f)(\widehat{w})= & \int_{T^{\star \mathbb{R}^{d}}} 2 i \lambda \mathcal{F}_{s}(\mathcal{P} f)(Y, \lambda) \overline{\mathcal{W}}(\widehat{w}, Y) \mathrm{d} Y \\
= & \int_{T^{\star} \mathbb{R}^{d}}\left(\mathcal{F}_{s} f(Y, \lambda)-\mathcal{F}_{s} f(Y,-\lambda)\right) \overline{\mathcal{W}}(\widehat{w}, Y) \mathrm{d} Y \\
= & \int_{T^{\star} \mathbb{R}^{d}} \mathcal{F}_{s} f(Y, \lambda) \overline{\mathcal{W}}(\widehat{w}, Y) \mathrm{d} Y \\
& \quad-(-1)^{|n+m|} \int_{T^{\star \mathbb{R}^{d}}} \mathcal{F}_{s} f(Y,-\lambda) \overline{\mathcal{W}}(m, n,-\lambda, Y) \mathrm{d} Y \\
= & \mathcal{F}_{\mathbb{H}} f(n, m, \lambda)-(-1)^{|n+m|} \mathcal{F}_{\mathbb{H}} f(m, n,-\lambda),
\end{aligned}
$$

which completes the proof of Lemma 2.4.

\subsection{Proof of the inversion theorem in the Schwartz space}

The aim of this section is to prove Theorem 2.6. To this end, let us first note that from Inequality (2.1) and Lemmas 2.3 and 2.4, we gather that $\mathcal{F}_{\mathbb{H}}$ maps $\mathcal{S}\left(\mathbb{H}^{d}\right)$ to $\mathcal{S}\left(\widehat{\mathbb{H}}^{d}\right)$ continuously. In addition, (2.8) guarantees that all elements of $\mathcal{S}\left(\widehat{\mathbb{H}}^{d}\right)$ are in $L^{1}\left(\widehat{\mathbb{H}}^{d}\right) \cap L^{2}\left(\widehat{\mathbb{H}}^{d}\right)$.

Hence Theorem 1.3 ensures that $\mathcal{F}_{\mathbb{H}}: \mathcal{S}\left(\mathbb{H}^{d}\right) \rightarrow \mathcal{S}\left(\widehat{\mathbb{H}}^{d}\right)$ is one-to-one, and that the inverse map has to be the functional $\widetilde{\mathcal{F}}_{\mathbb{H}}$ defined in (2.9). Therefore, there only remains to prove that $\widetilde{\mathcal{F}}_{\mathbb{H}}$ maps $\mathcal{S}\left(\widehat{\mathbb{H}}^{d}\right)$ to $\mathcal{S}\left(\mathbb{H}^{d}\right)$. To this end, it is convenient to introduce the semi-norms defined by:

$$
\|f\|_{K, \mathcal{S}\left(\mathbb{H}^{d}\right)}^{2} \stackrel{\text { def }}{=}\|f\|_{L^{2}\left(\mathbb{H}^{d}\right)}^{2}+\left\|M_{\mathbb{H}}^{K} f\right\|_{L^{2}\left(\mathbb{H}^{d}\right)}^{2}+\left\|\Delta_{\mathbb{H}}^{K} f\right\|_{L^{2}\left(\mathbb{H}^{d}\right)}^{2} \quad \text { with } \quad M_{\mathbb{H}} \stackrel{\text { def }}{=} M^{2}+M_{0}
$$

which are equivalent to the classical ones defined in Lemma 2.1 (see Proposition A.2). 
Let us compute $M^{2} \widetilde{\mathcal{F}}_{\mathbb{H}} \theta(Y, s)$. According to Lemma 3.1, we have for all $\widehat{w}=$ $(n, m, \lambda)$ in $\widetilde{\mathbb{H}}^{d}$,

$$
\begin{aligned}
& \sum_{(n, m) \in \mathbb{N}^{2 d}} \theta(\widehat{w})|Y|^{2} \mathcal{W}(\widehat{w}, Y) \\
&=\frac{1}{2|\lambda|} \sum_{(n, m) \in \mathbb{N}^{2 d}}\left((|n+m|+d) \mathcal{W}(\widehat{w}, Y) \theta(\widehat{w})-\sum_{j=1}^{d} \sqrt{n_{j} m_{j}} \theta(\widehat{w}) \mathcal{W}\left(\widehat{w}_{j}^{-}, Y\right)\right. \\
&\left.\quad-\sum_{j=1}^{d} \sqrt{\left(n_{j}+1\right)\left(m_{j}+1\right)} \theta(\widehat{w}) \mathcal{W}\left(\widehat{w}_{j}^{+}, Y\right)\right) .
\end{aligned}
$$

Changing variable $(\widetilde{n}, \widetilde{m})=\left(n+\delta_{j}, m+\delta_{j}\right)$ and $(\widetilde{n}, \widetilde{m})=\left(n-\delta_{j}, m-\delta_{j}\right)$, respectively, gives

$$
\begin{aligned}
& \sum_{(n, m) \in \mathbb{N}^{2 d}} \theta(\widehat{w})|Y|^{2} \mathcal{W}(\widehat{w}, Y) \\
& =\frac{1}{2|\lambda|} \sum_{(n, m) \in \mathbb{N}^{2 d}}((|n+m|+d) \theta(\widehat{w}) \mathcal{W}(\widehat{w}, Y) \\
& \left.\quad-\sum_{j=1}^{d}\left(\sqrt{\left(n_{j}+1\right)\left(m_{j}+1\right)} \theta\left(\widehat{w}_{j}^{+}\right)+\sqrt{n_{j} m_{j}} \theta\left(\widehat{w}_{j}^{-}\right)\right)\right) \mathcal{W}(\widehat{w}, Y) \\
& =-\sum_{(n, m) \in \mathbb{N}^{2 d}} \widehat{\Delta} \theta(\widehat{w}) \mathcal{W}(\widehat{w}, Y),
\end{aligned}
$$

where $\widehat{\Delta}$ is the operator introduced in $(2.2)$.

Multiplying by $2^{d-1} \pi^{-d-1} e^{i s \lambda}$, integrating with respect to $\lambda$ and remembering (2.9), we end up with

$$
\left(M^{2} \widetilde{\mathcal{F}}_{\mathbb{H}} \theta\right)(Y, s)=-\widetilde{\mathcal{F}}_{\mathbb{H}}(\widehat{\Delta} \theta)(Y, s) .
$$

Understanding how $M_{0}$ acts on $\widetilde{\mathcal{F}}_{\mathbb{H}}\left(\mathcal{S}\left(\widehat{\mathbb{H}}^{d}\right)\right)$ is more delicate. It requires using the continuity property of Definition 2.5. Now, if $\theta$ is in $\mathcal{S}\left(\widehat{\mathbb{H}}^{d}\right)$ then it is integrable. As obviously $|\mathcal{W}| \leqslant 1$, one may thus write for all $w=(Y, s)$ in $\mathbb{H}^{d}$, denoting $\mathbb{R}_{\varepsilon} \stackrel{\text { def }}{=}$ $\mathbb{R} \backslash[-\varepsilon, \varepsilon]$,

$$
\begin{gathered}
\left(M_{0} \widetilde{\mathcal{F}}_{\mathbb{H}} \theta\right)(w)=\frac{2^{d-1}}{\pi^{d+1}} \lim _{\varepsilon \rightarrow 0} \sum_{(n, m) \in \mathbb{N}^{2 d}} \Psi_{\varepsilon}(n, m, w) \\
\text { with } \quad \Psi_{\varepsilon}(n, m, w) \stackrel{\text { def }}{=}-\int_{\mathbb{R}_{\varepsilon}}\left(\frac{\mathrm{d}}{\mathrm{d} \lambda} e^{i s \lambda}\right) \theta(n, m, \lambda) \mathcal{W}(n, m, \lambda, Y)|\lambda|^{d} \mathrm{~d} \lambda .
\end{gathered}
$$

Integrating by parts yields

$$
\begin{aligned}
\Psi_{\varepsilon}(n, m, w) & =\Psi_{\varepsilon}^{(1)}(n, m, w)-\Psi_{\varepsilon}^{(2)}(n, m, w) \text { with } \\
\Psi_{\varepsilon}^{(1)}(n, m, w) & \stackrel{\text { def }}{=} \int_{\mathbb{R}_{\varepsilon}} e^{i s \lambda} \frac{\mathrm{d}}{\mathrm{d} \lambda}\left(\mathcal{W}(n, m, \lambda, Y) \theta(n, m, \lambda)|\lambda|^{d}\right) \mathrm{d} \lambda \text { and } \\
\Psi_{\varepsilon}^{(2)}(n, m, w) & \stackrel{\text { def }}{=} \varepsilon^{d}\left(e^{i s \varepsilon} \mathcal{W}(n, m, \varepsilon, Y) \theta(n, m, \varepsilon)-e^{-i s \varepsilon} \mathcal{W}(n, m,-\varepsilon, Y) \theta(n, m,-\varepsilon)\right) .
\end{aligned}
$$


Let us compute

$$
\Theta(\widehat{w}, Y) \stackrel{\text { def }}{=} \frac{\mathrm{d}}{\mathrm{d} \lambda}\left(\mathcal{W}(n, m, \lambda, Y) \theta(n, m, \lambda)|\lambda|^{d}\right) .
$$

Leibniz's rule gives

$$
\Theta(\widehat{w}, Y)=\partial_{\lambda} \mathcal{W}(\widehat{w}, Y) \theta(\widehat{w})|\lambda|^{d}+\mathcal{W}(\widehat{w}, Y) \frac{\mathrm{d}}{\mathrm{d} \lambda}\left(|\lambda|^{d} \theta(\widehat{w})\right)
$$

Hence, remembering Identity (3.1), we discover that

$$
\begin{aligned}
\Theta(\widehat{w}, Y)= & \frac{\mathrm{d} \theta}{\mathrm{d} \lambda}(\widehat{w}) \mathcal{W}(\widehat{w}, Y)|\lambda|^{d}+\frac{\mathrm{d}}{2 \lambda} \theta(\widehat{w}) \mathcal{W}(\widehat{w}, Y)|\lambda|^{d} \\
& -\frac{|\lambda|^{d}}{2 \lambda} \sum_{j=1}^{d} \theta(\widehat{w})\left(\sqrt{n_{j} m_{j}} \mathcal{W}\left(\widehat{w}_{j}^{-}, \lambda, Y\right)-\sqrt{\left(n_{j}+1\right)\left(m_{j}+1\right)} \mathcal{W}\left(\widehat{w}_{j}^{+}, Y\right)\right) .
\end{aligned}
$$

From the changes of variable $\left(n^{\prime}, m^{\prime}\right)=\left(n-\delta_{j}, m-\delta_{j}\right)$ and $(\widetilde{n}, \widetilde{m})=\left(n+\delta_{j}, m+\delta_{j}\right)$, we infer that

$$
\begin{aligned}
\sum_{(n, m) \in \mathbb{N}^{2 d}} \theta(\widehat{w}) & \left(\sqrt{n_{j} m_{j}} \mathcal{W}\left(\widehat{w}_{j}^{-}, Y\right)-\sqrt{\left(n_{j}+1\right)\left(m_{j}+1\right)} \mathcal{W}\left(\widehat{w}_{j}^{+}, Y\right)\right) \\
= & -\sum_{(n, m) \in \mathbb{N}^{2 d}} \mathcal{W}(\widehat{w}, Y)\left(\sqrt{n_{j} m_{j}} \theta\left(\widehat{w}_{j}^{-}\right)-\sqrt{\left(n_{j}+1\right)\left(m_{j}+1\right)} \theta\left(\widehat{w}_{j}^{+}\right)\right) .
\end{aligned}
$$

Therefore, using the operator $\widehat{\mathcal{D}}_{\lambda}$ introduced in Definition 2.2, we get

$$
\sum_{(n, m) \in \mathbb{N}^{2 d}} \Psi_{\varepsilon}^{(1)}(n, m, w)=\sum_{(n, m) \in \mathbb{N}^{2 d}} \int_{\mathbb{R}_{\varepsilon}} e^{i s \lambda}\left(\widehat{\mathcal{D}}_{\lambda} \theta\right)(n, m, \lambda) \mathcal{W}(n, m, \lambda, Y)|\lambda|^{d} \mathrm{~d} \lambda
$$

Since $\widehat{\mathcal{D}}_{\lambda} \theta$ belongs to $\mathcal{S}\left(\widehat{\mathbb{H}}^{d}\right)$ and is thus integrable, Lebesgue dominated convergence theorem guarantees that

$$
\lim _{\varepsilon \rightarrow 0} \sum_{(n, m) \in \mathbb{N}^{2 d}} \Psi_{\varepsilon}^{(1)}(n, m, w)=\int_{\widehat{\mathbb{H}} d} e^{i s \lambda}\left(\widehat{\mathcal{D}}_{\lambda} \theta\right)(\widehat{w}) \mathcal{W}(\widehat{w}, Y) \mathrm{d} \widehat{w}
$$

In order to show that the contribution of $\Psi_{\varepsilon}^{(2)}(n, m, w)$ tends to 0 , the boundedness of $\widehat{\Sigma}_{0} \theta$ will come into play. Indeed, we have

$$
\begin{aligned}
e^{i s \varepsilon} \mathcal{W}(n, m, \varepsilon, Y) \theta(n, m, \varepsilon)-e^{-i s \varepsilon} \mathcal{W}(n, m,-\varepsilon, Y) \theta(n, m,-\varepsilon) \\
=\left(e^{i s \varepsilon}-e^{-i s \varepsilon}\right) \mathcal{W}(n, m, \varepsilon, Y) \theta(n, m, \varepsilon) \\
\quad+e^{-i s \varepsilon}(\mathcal{W}(n, m, \varepsilon, Y) \theta(n, m, \varepsilon)-\mathcal{W}(n, m,-\varepsilon, Y) \theta(n, m,-\varepsilon)) .
\end{aligned}
$$


Hence, thanks to $(2.7)$

$$
\begin{aligned}
\sum_{(n, m) \in \mathbb{N}^{2 d}} \Psi_{\varepsilon}^{(2)}(n, m, w) & =2 i \varepsilon^{d} \sin (s \varepsilon) \sum_{(n, m) \in \mathbb{N}^{2 d}} \mathcal{W}(n, m, \varepsilon, Y) \theta(n, m, \varepsilon) \\
+\varepsilon^{d} e^{-i s \varepsilon}( & \sum_{(n, m) \in \mathbb{N}^{2 d}} \mathcal{W}(n, m, \varepsilon, Y) \theta(n, m, \varepsilon) \\
& \left.-\sum_{(n, m) \in \mathbb{N}^{2 d}}(-1)^{|n+m|} \mathcal{W}(m, n, \varepsilon, Y) \theta(n, m,-\varepsilon)\right) .
\end{aligned}
$$

Swapping indices $n$ and $m$ in the last sum gives

$$
\begin{aligned}
\sum_{(n, m) \in \mathbb{N}^{2 d}} \Psi_{\varepsilon}^{(2)}(n, m, w)=2 i \varepsilon^{d} \sin (s \varepsilon) & \sum_{(n, m) \in \mathbb{N}^{2 d}} \mathcal{W}(n, m, \varepsilon, Y) \theta(n, m, \varepsilon) \\
& +\varepsilon^{d+1} e^{-i s \varepsilon} \sum_{(n, m) \in \mathbb{N}^{2 d}} \mathcal{W}(n, m, \varepsilon, Y)\left(\widehat{\Sigma}_{0} \theta\right)(n, m, \varepsilon) .
\end{aligned}
$$

Remembering that $|\mathcal{W}| \leqslant 1$, we thus get

$$
\begin{aligned}
& \left|\sum_{(n, m) \in \mathbb{N}^{2 d}} \Psi_{\varepsilon}^{(2)}(n, m, w)\right| \\
& \leqslant \varepsilon^{d+1}\left(2|s| \sum_{(n, m) \in \mathbb{N}^{2 d}}|\theta(n, m, \varepsilon)|+\sum_{(n, m) \in \mathbb{N}^{2 d}}\left|\left(\widehat{\Sigma}_{0} \theta\right)(n, m, \varepsilon)\right|\right) .
\end{aligned}
$$

Since

$$
\sum_{(n, m) \in \mathbb{N}^{2 d}}|\theta(n, m, \varepsilon)| \leqslant\|\theta\|_{2 d+2,0, \mathcal{S}\left(\widetilde{\mathbb{H}}^{d}\right)} \sum_{(n, m) \in \mathbb{N}^{2 d}}(1+\varepsilon(|n+m|+d)+|n-m|)^{-2 d-2}
$$

and

$$
\begin{aligned}
\sum_{(n, m) \in \mathbb{N}^{2 d}}(1+\varepsilon(|n+m|+d)+|n-m|)^{-2 d-2} & \leqslant \sum_{\ell \in \mathbb{N}^{d}}(1+\varepsilon(|\ell|+d))^{-d-1} \sum_{k \in \mathbb{Z}^{d}}(1+|k|)^{-d-1} \\
& \leqslant C \varepsilon^{-d}
\end{aligned}
$$

the first term of the right-hand side of (3.6) tends to 0 when $\varepsilon$ goes to 0. Employing the same argument with $\widehat{\Sigma}_{0} \theta$ guarantees that we do have

$$
\lim _{\varepsilon \rightarrow 0} \sum_{(n, m) \in \mathbb{N}^{2 d}} \Psi_{\varepsilon}^{(2)}(n, m, w)=0,
$$

from which one may conclude that

$$
M_{0} \widetilde{\mathcal{F}}_{\mathbb{H}} \theta=\widetilde{\mathcal{F}}_{\mathbb{H}} \widehat{\mathcal{D}}_{\lambda} \theta
$$

Together with (3.4), this implies that

$$
M_{\mathbb{H}} \widetilde{\mathcal{F}}_{\mathbb{H}} \theta=\widetilde{\mathcal{F}}_{\mathbb{H}}\left(\left(-\widehat{\Delta}+\widehat{\mathcal{D}}_{\lambda}\right)(\theta)\right) \quad \text { with } \quad M_{\mathbb{H}} \stackrel{\text { def }}{=} M^{2}+M_{0} .
$$

Hence, for any integer $K$, there exist an integer $N_{K}$ and a constant $C_{K}$ so that

$$
\left\|M_{\mathbb{H}}^{K} \widetilde{\mathcal{F}} \theta\right\|_{L^{2}\left(\mathbb{H}^{d}\right)} \leqslant C_{K}\|\theta\|_{N_{K}, N_{K}, \mathcal{S}\left(\widehat{\mathbb{H}}^{d}\right)} .
$$


Finally, to study the action of the sublaplacian on $\widetilde{\mathcal{F}}_{\mathbb{H}}\left(\mathcal{S}\left(\widehat{\mathbb{H}}^{d}\right)\right)$, we write that by definition of $\mathcal{X}_{j}$ and of $\mathcal{W}$, we have

$$
\begin{aligned}
\mathcal{X}_{j}\left(e^{i s \lambda} \mathcal{W}(\widehat{w}, Y)\right) & =\int_{\mathbb{R}^{d}} \mathcal{X}_{j}\left(e^{i s \lambda+2 i \lambda\langle\eta, z\rangle} H_{n, \lambda}(y+z) H_{m, \lambda}(-y+z)\right) \mathrm{d} z \\
& =\int_{\mathbb{R}^{d}} e^{i s \lambda+2 i \lambda\langle\eta, z\rangle}\left(2 i \lambda \eta_{j}+\partial_{y_{j}}\right)\left(H_{n, \lambda}(y+z) H_{m, \lambda}(-y+z)\right) \mathrm{d} z .
\end{aligned}
$$

As $2 i \lambda \eta_{j} e^{2 i \lambda\langle\eta, z\rangle}=\partial_{z_{j}}\left(e^{2 i \lambda\langle\eta, z\rangle}\right)$, integrating by parts yields

$$
\mathcal{X}_{j}\left(e^{i s \lambda} \mathcal{W}(\widehat{w}, Y)\right)=\int_{\mathbb{R}^{d}} e^{i s \lambda+2 i \lambda\langle\eta, z\rangle}\left(\partial_{y_{j}}-\partial_{z_{j}}\right)\left(H_{n, \lambda}(y+z) H_{m, \lambda}(-y+z)\right) \mathrm{d} z
$$

The action of $\Xi_{j}$ is simply described by

$$
\begin{aligned}
\Xi_{j}\left(e^{i s \lambda} \mathcal{W}(\widehat{w}, Y)\right) & =\int_{\mathbb{R}^{d}} \Xi_{j}\left(e^{i s \lambda+2 i \lambda\langle\eta, z\rangle}\right) H_{n, \lambda}(y+z) H_{m, \lambda}(-y+z) \mathrm{d} z \\
& =\int_{\mathbb{R}^{d}} e^{i s \lambda+2 i \lambda\langle\eta, z\rangle} 2 i \lambda\left(z_{j}-y_{j}\right) H_{n, \lambda}(y+z) H_{m, \lambda}(-y+z) \mathrm{d} z .
\end{aligned}
$$

Together with (3.8) and the definition of $\Delta_{\mathbb{H}}$ in (1.6), this gives

$$
\begin{aligned}
\Delta_{\mathbb{H}}\left(e^{i s \lambda} \mathcal{W}(\widehat{w}, Y)\right) & =4 \int_{\mathbb{R}^{d}} e^{i s \lambda+2 i \lambda\langle\eta, z\rangle} H_{n, \lambda}(y+z)\left(\Delta_{o s c}^{\lambda} H_{m, \lambda}\right)(-y+z) \mathrm{d} z \\
& =-4|\lambda|(2|m|+d) e^{i s \lambda} \mathcal{W}(\widehat{w}, Y) .
\end{aligned}
$$

This implies that for all integers $K$, we have

$$
\left(-\Delta_{\mathbb{H}}\right)^{K}\left(\widetilde{\mathcal{F}}_{\mathbb{H}} \theta\right)=\widetilde{\mathcal{F}}_{\mathbb{H}} \widehat{M}^{K} \theta \quad \text { with } \quad \widehat{M} \theta(n, m, \lambda) \stackrel{\text { def }}{=} 4|\lambda|(2|m|+d) \theta(n, m, \lambda),
$$

whence there exist an integer $N_{K}$ and a constant $C_{K}$ so that

$$
\left\|\left(-\Delta_{\mathbb{H}}\right)^{K}\left(\widetilde{\mathcal{F}}_{\mathbb{H}} \theta\right)\right\|_{L^{2}\left(\mathbb{H}^{d}\right)} \leqslant C_{K}\|\theta\|_{N_{K}, N_{K}, \mathcal{S}\left(\widehat{\mathbb{H}}^{d}\right)} .
$$

Putting (3.7) and (3.9) together and remembering the definition of the semi-norms on $\mathcal{S}\left(\mathbb{H}^{d}\right)$ given in $(3.3)$, we conclude that for all integer $K$, there exist an integer $N_{K}$ and a constant $C_{K}$ so that

$$
\left\|\widetilde{\mathcal{F}}_{\mathbb{H}} \theta\right\|_{K, \mathcal{S}\left(\mathbb{H}^{d}\right)} \leqslant C_{K}\|\theta\|_{N_{K}, N_{K}, \mathcal{S}\left(\widehat{\mathbb{H}}^{d}\right)} .
$$

This completes the proof of Theorem 2.6.

\section{Examples of functions in the range of the Schwartz class}

The purpose of this sectionis to prove Theorem 2.8. Let us recall the notation

$$
\Theta_{f}(\widehat{w}) \stackrel{\text { def }}{=} f(|\lambda| R(n, m), m-n, \lambda) \quad \text { with } \quad R(n, m) \stackrel{\text { def }}{=}\left(n_{j}+m_{j}+1\right)_{1 \leqslant j \leqslant d} .
$$

For any function $f$ in $\mathcal{S}_{d}^{+}$which is either supported in $\left[0, \infty\left[^{d} \times\{0\} \times \mathbb{R}\right.\right.$ or in $\left[r_{0}, \infty\left[^{d} \times \mathbb{Z}^{d} \times \mathbb{R}\right.\right.$ for some positive real number $r_{0}$ and satisfies (2.11), the fact that $\left\|\Theta_{f}\right\|_{N, 0, \mathcal{S}\left(\widehat{\mathbb{H}}^{d}\right)}$ is finite for all integer $N$ is obvious. We next have to study the action of $\widehat{\Delta}$ and $\widehat{\mathcal{D}}_{\lambda}$ on $\Theta_{f}$. To this end, we shall establish a Taylor type expansion of $\widehat{\Delta} \Theta_{f}$ and $\widehat{\mathcal{D}}_{\lambda} \Theta_{f}$ near $\lambda=0$. To explain what kind of convergence we are looking for, we need the following definition. 
Definition 4.1. - Let $M$ be an integer. We say that two continuous functions $\theta$ and $\theta^{\prime}$ on $\widehat{\mathbb{H}}^{d}$ are $M$-equivalent (denoted by $\theta \stackrel{M}{\equiv} \theta^{\prime}$ ) if for all positive integer $N$, a constant $C_{N, M}$ exists such that

$$
\forall \widehat{w} \in \widetilde{\mathbb{H}}^{d},\left|\theta(\widehat{w})-\theta^{\prime}(\widehat{w})\right| \leqslant C_{N, M}|\lambda|^{M}(1+|\lambda|(|n+m|+d)+|m-n|)^{-N} .
$$

Obviously, if two continuous functions $\theta$ and $\theta^{\prime}$ on $\widetilde{\mathbb{H}}^{d}$ are $M$-equivalent for some $M \geqslant 1$ then they are also $(M-1)$-equivalent, and $\widehat{\Sigma}_{0} \theta$ is $(M-1)$-equivalent to $\widehat{\Sigma}_{0} \theta^{\prime}$. Hence

$$
\theta \stackrel{M}{=} 0 \Longrightarrow\|\theta\|_{N, 0}<\infty \text { for all } N \in \mathbb{N} \text {. }
$$

It is also clear that

$$
\theta_{1} \stackrel{M}{=} \theta_{1}^{\prime} \quad \text { and } \quad \theta_{2} \stackrel{M}{\equiv} \theta_{2}^{\prime} \text { implies } \theta_{1}+\theta_{2} \stackrel{M}{=} \theta_{1}^{\prime}+\theta_{2}^{\prime}
$$

that, whenever $0 \leqslant M_{0} \leqslant M$ and $\lambda \neq 0$, we have

$$
\theta \stackrel{M}{\equiv} \theta^{\prime} \Longrightarrow|\lambda|^{-M_{0}} \theta \stackrel{M-M_{0}}{\equiv}|\lambda|^{-M_{0}} \theta^{\prime}
$$

and that, if the function $P$ is bounded by a polynomial in $(n, m)$ with degree $M_{0}$, then

$$
\theta \stackrel{M}{\equiv} \theta^{\prime} \Longrightarrow P(n, m) \theta^{M-M_{0}} P(n, m) \theta^{\prime} .
$$

Finally, the definition of $\widehat{\Delta}$ in (2.2) implies that

$$
\theta \stackrel{M}{\equiv} \theta^{\prime} \Longrightarrow \widehat{\Delta} \theta \stackrel{M-2}{\equiv} \widehat{\Delta} \theta^{\prime} \text { and } \quad \widehat{\mathcal{D}}_{\lambda} \theta \stackrel{M-2}{\equiv} \widehat{\mathcal{D}}_{\lambda} \theta^{\prime}
$$

We have the following lemma.

Lemma 4.2. - For any positive integer $M$, we have

$$
\forall \widehat{w} \in \widetilde{\mathbb{H}}^{d}, \Theta_{f}\left(\widehat{w}_{j}^{ \pm}\right) \stackrel{M+1}{\equiv} \sum_{\ell=0}^{M} \frac{( \pm 2|\lambda|)^{\ell}}{\ell !} \Theta_{\partial_{x_{j}} f}(\widehat{w}) .
$$

Proof. - Performing a Taylor expansion at order $M+1$, we get

$$
\begin{aligned}
& f\left(|\lambda| R\left(n \pm \delta_{j}, m \pm \delta_{j}\right), m-n, \lambda\right) \\
& =\sum_{\ell=0}^{M} \frac{( \pm 2|\lambda|)^{\ell}}{\ell !}\left(\partial_{x_{j}}^{\ell} f\right)(|\lambda| R(n, m), m-n, \lambda) \\
& \quad+( \pm 2|\lambda|)^{M+1} \int_{0}^{1} \frac{(1-t)^{M}}{M !}\left(\partial_{x_{j}}^{M+1} f\right)\left(|\lambda| R_{j}^{ \pm}(n, m, t), m-n, \lambda\right) \mathrm{d} t
\end{aligned}
$$

with $R_{j}^{ \pm}(n, m, t) \stackrel{\text { def }}{=}\left(n_{1}+m_{1}+1, \ldots, n_{j}+m_{j}+1 \pm 2 t, \ldots, n_{d}+m_{d}+1\right)$. The fact that $f$ belongs to $\mathcal{S}_{d}^{+}$implies that for any positive integer $N$, we have

$$
\begin{aligned}
\mid \int_{0}^{1} \frac{(1-t)^{M}}{M !} \partial_{x_{j}}^{M+1} f\left(|\lambda| R_{j}^{+}(n, m, t), m\right. & -n, \lambda) \mathrm{d} t \mid \\
& \leqslant C_{N}(1+|\lambda|(|n+m|+d)+|m-n|)^{-N} .
\end{aligned}
$$

This gives the lemma. 
One can now tackle the proof of Theorem 2.8. Let us first investigate the (easier) case when the support of $f$ is included in $\left[0, \infty\left[^{d} \times\{0\} \times \mathbb{R}\right.\right.$. The first step consists in computing an equivalent (in the sense of Definition 4.1) of $\widehat{\Delta} \Theta_{f}$ at an order which will be chosen later on. For notational simplicity, we here set $R(n) \stackrel{\text { def }}{=} R(n, n)$ and omit the second variable of $f$. Now, by definition of the operator $\widehat{\Delta}$, we have

$$
\begin{aligned}
& \left(-\widehat{\Delta} \Theta_{f}\right)(n, n, \lambda)=\frac{1}{2|\lambda|}\left((|2 n|+d) f(|\lambda| R(n), \lambda)-\sum_{j=1}^{d} \widetilde{\Delta}_{j}(n, \lambda)\right) \\
& \quad \text { with } \quad \widetilde{\Delta}_{j}(n, \lambda) \stackrel{\text { def }}{=}\left(n_{j}+1\right) f\left(|\lambda| R\left(n+2 \delta_{j}\right), \lambda\right)+n_{j} f\left(|\lambda| R\left(n-2 \delta_{j}\right), \lambda\right) .
\end{aligned}
$$

Lemma 4.2, and Assertions (4.3) and (4.4) imply that

$$
\begin{aligned}
\frac{1}{2|\lambda|} \widetilde{\Delta}_{j}(n, \lambda) \stackrel{2 M-1}{\equiv} \frac{n_{j}+1}{2|\lambda|} \sum_{\ell=0}^{2 M} \frac{(2|\lambda|)^{\ell}}{\ell !}\left(\partial_{x_{j}}^{\ell} f\right)(|\lambda| R(n), \lambda) \\
+\frac{n_{j}}{2|\lambda|} \sum_{\ell=0}^{2 M} \frac{(-2|\lambda|)^{\ell}}{\ell !}\left(\partial_{x_{j}}^{\ell} f\right)(|\lambda| R(n), \lambda) \\
\stackrel{2 M-1}{\equiv} \frac{2 n_{j}+1}{2|\lambda|} \sum_{\ell=0}^{M} \frac{(2 \lambda)^{2 \ell}}{(2 \ell) !}\left(\partial_{x_{j}}^{2 \ell} f\right)(|\lambda| R(n), \lambda) \\
+\frac{1}{2|\lambda|} \sum_{\ell=0}^{M-1} \frac{(2|\lambda|)^{2 \ell+1}}{(2 \ell+1) !}\left(\partial_{x_{j}}^{2 \ell+1} f\right)(|\lambda| R(n), \lambda) .
\end{aligned}
$$

Let us define

$$
\begin{array}{r}
f_{2 \ell}(x, \lambda) \stackrel{\text { def }}{=} \sum_{j=1}^{d} \frac{2^{2 \ell-1}}{(2 \ell) !} x_{j} \lambda^{2 \ell-2} \partial_{x_{j}}^{2 \ell} f(x, \lambda) \\
\text { and } f_{2 \ell+1}(x, \lambda) \stackrel{\text { def }}{=} \sum_{j=1}^{d} \frac{2^{2 \ell}}{(2 \ell+1) !} \lambda^{2 \ell} \partial_{x_{j}}^{2 \ell+1} f(x, \lambda) .
\end{array}
$$

Clearly, all functions $f_{\ell}$ are supported in $\left[0, \infty\left[^{d} \times\{0\} \times \mathbb{R}\right.\right.$ and belong to $\mathcal{S}_{+}^{d}$, and the above equality rewrites

$$
\widehat{\Delta} \Theta_{f}(n, n, \lambda) \stackrel{2 M-1}{\equiv}-\sum_{\ell=1}^{2 M} f_{\ell}(|\lambda| R(n), \lambda) .
$$

Arguing by induction, it is easy to establish that for any function $f$ in $\mathcal{S}_{d}^{+}$supported in $\left[0, \infty\left[\times\{0\} \times \mathbb{R}\right.\right.$ and any integers $N$ and $p$, the quantity $\left\|\widehat{\Delta}^{p} \Theta_{f}\right\|_{N, 0, \mathcal{S}\left(\widehat{\mathbb{H}}^{d}\right)}$ is finite. Indeed, this is obvious for $p=0$. Now, if the property holds true for some non negative integer $p$ then, thanks to (4.5) and (4.7),

$$
\widehat{\Delta}^{p+1} \Theta_{f}(n, n, \lambda) \stackrel{2 M-1-2 p}{\equiv}-\sum_{\ell=1}^{2 M} \widehat{\Delta}^{p} \Theta_{f_{\ell}}(n, \lambda) .
$$

From (4.1), (4.7) and the induction hypothesis, it is clear that if we choose $M$ greater than $p$ then we get that $\left\|\widehat{\Delta}^{p} \Theta_{f}\right\|_{N, 0, \mathcal{S}\left(\widehat{\mathbb{H}}^{d}\right)}$ is finite for all integer $N$. 
Let us next study the action of Operator $\widehat{\mathcal{D}}_{\lambda}$. From its definition in Lemma 2.3 and the chain rule, we gather that

$$
\begin{aligned}
\left(\widehat{\mathcal{D}}_{\lambda} \Theta_{f}\right)(n, n, \lambda)= & \left(\partial_{\lambda} f\right)(|\lambda| R(n), \lambda)+\frac{d}{2 \lambda} f(|\lambda| R(n), \lambda) \\
& +\operatorname{sgn} \lambda \sum_{j=1}^{d}\left(2 n_{j}+1\right)\left(\partial_{x_{j}} f\right)(|\lambda| R(n), \lambda)+\frac{1}{2 \lambda} \sum_{j=1}^{d} \mathcal{D}_{j}(n, \lambda)
\end{aligned}
$$

with $\mathcal{D}_{j}(n, \lambda) \stackrel{\text { def }}{=} n_{j} f\left(|\lambda|\left(R(n)-2 \delta_{j}\right), \lambda\right)-\left(n_{j}+1\right) f\left(|\lambda|\left(R(n)+2 \delta_{j}\right), \lambda\right)$.

Lemma 4.2, and Assertions (4.3) and (4.4) imply that

$$
\begin{aligned}
& \frac{1}{2 \lambda} \mathcal{D}_{j}(n, \lambda) \stackrel{2 M-1}{\equiv}-\frac{n_{j}+1}{2 \lambda} \sum_{\ell=0}^{2 M} \frac{(2|\lambda|)^{\ell}}{\ell !}\left(\partial_{x_{j}}^{\ell} f\right)(|\lambda| R(n), \lambda) \\
& +\frac{n_{j}}{2 \lambda} \sum_{\ell=0}^{2 M} \frac{(-2|\lambda|)^{\ell}}{\ell !}\left(\partial_{x_{j}}^{\ell} f\right)(|\lambda| R(n), \lambda) \\
& \stackrel{2 M-1}{\equiv}-\sum_{\ell=0}^{M} \frac{(2 \lambda)^{2 \ell-1}}{(2 \ell) !}\left(\partial_{x_{j}}^{2 \ell} f\right)(|\lambda| R(n), \lambda) \\
& -\frac{2 n_{j}+1}{2 \lambda} \sum_{\ell=0}^{M-1} \frac{(2|\lambda|)^{2 \ell+1}}{(2 \ell+1) !}\left(\partial_{x_{j}}^{2 \ell+1} f\right)(|\lambda| R(n), \lambda) .
\end{aligned}
$$

Observe that the term corresponding to $\ell=0$ of the first and second sums above cancel out with the second and third terms of the right-hand side of (4.8), respectively. Hence, defining for $\ell \geqslant 1$ the functions

$$
\begin{gathered}
\widetilde{f}_{2 \ell}(x, \lambda) \stackrel{\text { def }}{=} \sum_{j=1}^{d} \frac{2^{2 \ell-1}}{(2 \ell) !} \lambda^{2 \ell-1} \partial_{x_{j}}^{2 \ell} f(x, \lambda) \\
\text { and } \widetilde{f}_{2 \ell+1}(x, \lambda) \stackrel{\text { def }}{=} \sum_{j=1}^{d} \frac{2^{2 \ell}}{(2 \ell+1) !} x_{j} \lambda^{2 \ell-1} \partial_{x_{j}}^{2 \ell+1} f(x, \lambda),
\end{gathered}
$$

we get, using (4.8) and (4.9),

$$
\left(\widehat{\mathcal{D}}_{\lambda} \Theta_{f}\right)(n, n, \lambda)-\left(\Theta_{\partial_{\lambda} f}\right)(n, n, \lambda) \stackrel{2 M-1}{\equiv}-\sum_{\ell=2}^{2 M} \widetilde{f}_{\ell}(|\lambda| R(n), \lambda) .
$$

From that relation, mimicking the induction proof for $\widehat{\Delta}$, we easily conclude that for any function $f$ in $\mathcal{S}_{d}^{+}$supported in $\left[0, \infty\left[{ }^{d} \times\{0\} \times \mathbb{R}\right.\right.$, and any integer $p$, the quantity $\left\|\widehat{\mathcal{D}}_{\lambda}^{p} \Theta_{f}\right\|_{N, 0, \mathcal{S}\left(\widehat{\mathbb{H}}^{d}\right)}$ is finite for all integer $N$. This completes the proof Theorem 2.8 in that particular case.

Next, let us investigate the case when the function $f$ of $\mathcal{S}_{d}^{+}$is supported in the set $\left[r_{0}, \infty\left[^{d} \times \mathbb{Z}^{d} \times \mathbb{R}\right.\right.$ for some positive $r_{0}$ and satisfies (2.11). Then, by definition of 
the operator $\widehat{\Delta}$, we have for all $\widehat{w}=(n, m, \lambda)$ in $\widetilde{\mathbb{H}}^{d}$, denoting $k \stackrel{\text { def }}{=} m-n$,

$$
-\widehat{\Delta} \Theta_{f}(\widehat{w}) \stackrel{\text { def }}{=} \frac{1}{2|\lambda|}\left((|n+m|+d) f(|\lambda| R(n, m), k, \lambda)-\sum_{j=1}^{d} \widetilde{\Delta}_{j}(\widehat{w})\right)
$$

with $\quad \widetilde{\Delta}_{j}(\widehat{w}) \stackrel{\text { def }}{=} \sqrt{\left(n_{j}+1\right)\left(m_{j}+1\right)} f\left(|\lambda|\left(R(n, m)+2 \delta_{j}\right), k, \lambda\right)$

$$
+\sqrt{n_{j} m_{j}} f\left(|\lambda|\left(R(n, m)-2 \delta_{j}\right), k, \lambda\right) .
$$

Compared to (4.6), the computations get wilder, owing to the square roots in the above formula. Let $M$ be an integer (to be suitably chosen later on). Lemma 4.2, and Assertions (4.3) and (4.4) imply that

$$
\begin{aligned}
\frac{1}{2|\lambda|} \widetilde{\Delta}_{j}(\widehat{w}) \stackrel{2 M-1}{\equiv} \frac{\sqrt{\left(n_{j}+1\right)\left(m_{j}+1\right)}}{2|\lambda|} \sum_{\ell=0}^{2 M} \frac{(2|\lambda|)^{\ell}}{\ell !}\left(\partial_{x_{j}}^{\ell} f\right)(|\lambda| R(n, m), k, \lambda) \\
+\frac{\sqrt{n_{j} m_{j}}}{2|\lambda|} \sum_{\ell=0}^{2 M} \frac{(-2|\lambda|)^{\ell}}{\ell !}\left(\partial_{x_{j}}^{\ell} f\right)(|\lambda| R(n, m), k, \lambda) .
\end{aligned}
$$

Defining

$$
\alpha^{ \pm}(p, q) \stackrel{\text { def }}{=} \sqrt{(p+1)(q+1)} \pm \sqrt{p q}
$$

for nonnegative integers $p$ and $q$, we get

$$
\frac{1}{2|\lambda|} \widetilde{\Delta}_{j}(\widehat{w}) \stackrel{2 M-1}{\equiv} \widetilde{\Delta}_{j}^{0}(\widehat{w})+\widetilde{\Delta}_{j}^{1}(\widehat{w})
$$

with $\quad \widetilde{\Delta}_{j}^{0}(\widehat{w}) \stackrel{\text { def }}{=} \frac{\alpha^{+}\left(n_{j}, m_{j}\right)}{2|\lambda|} \sum_{\ell=0}^{M} \frac{(2 \lambda)^{2 \ell}}{(2 \ell) !}\left(\partial_{x_{j}}^{2 \ell} f\right)(|\lambda| R(n, m), k, \lambda)$

$$
\text { and } \quad \widetilde{\Delta}_{j}^{1}(\widehat{w}) \stackrel{\text { def }}{=} \frac{\alpha^{-}\left(n_{j}, m_{j}\right)}{2|\lambda|} \sum_{\ell=0}^{M-1} \frac{(2|\lambda|)^{2 \ell+1}}{(2 \ell+1) !}\left(\partial_{x_{j}}^{2 \ell+1} f\right)(|\lambda| R(n, m), k, \lambda) .
$$

In order to compute an expansion of $\alpha_{j}^{ \pm}(n, m)$ with respect to $n_{j}+m_{j}+1$ and $n_{j}-m_{j}$, we shall take advantage of the following two identities that are valid for any $(p, q)$ in $\mathbb{N}^{2}$ :

$$
\begin{aligned}
\sqrt{(p+1)(q+1)} & =\frac{1}{2}(p+q+1) \sqrt{1+\frac{2}{p+q+1}+\frac{1-(p-q)^{2}}{(p+q+1)^{2}}} \\
\text { and } \sqrt{p q} & =\frac{1}{2}(p+q+1) \sqrt{1-\frac{2}{p+q+1}+\frac{1-(p-q)^{2}}{(p+q+1)^{2}}} .
\end{aligned}
$$

Let us introduce the notation $f(p, q)=\mathcal{O}_{M}(p, q)$ to mean that for some constant $C$, there holds

$$
|f(p, q)| \leqslant C\left(\frac{1}{(p+q+1)^{M}}+\frac{|p-q|^{2 M+2}}{(p+q+1)^{2 M+1}}\right) .
$$

Using the following Taylor expansion with $K=2 M$ :

$$
\sqrt{1+u}=1+\sum_{\ell_{1}=1}^{K} a_{\ell_{1}} u^{\ell_{1}}+(K+1) a_{K+1} u^{K+1} \int_{0}^{1}(1+t u)^{-K-\frac{1}{2}}(1-t)^{K} \mathrm{~d} t,
$$


we gather that, up to a $\mathcal{O}_{2 M}(p, q)$ term, we have

$$
\begin{aligned}
\sqrt{(p+1)(q+1)} & =\frac{1}{2}(p+q+1)\left(1+\sum_{\ell_{1}=1}^{2 M} a_{\ell_{1}}\left(\frac{2}{p+q+1}+\frac{1-(p-q)^{2}}{(p+q+1)^{2}}\right)^{\ell_{1}}\right) \\
\text { and } \sqrt{p q} & =\frac{1}{2}(p+q+1)\left(1+\sum_{\ell_{1}=1}^{2 M} a_{\ell_{1}}\left(-\frac{2}{p+q+1}+\frac{1-(p-q)^{2}}{(p+q+1)^{2}}\right)^{\ell_{1}}\right) .
\end{aligned}
$$

Now we can compute the expansion of $\alpha^{ \pm}(p, q)$. Newton's formula gives

$$
\begin{aligned}
& \alpha^{+}(p, q)=p+q+1+\sum_{\substack{1 \leqslant \ell_{1} \leqslant 2 M \\
2 \ell_{2} \leqslant \ell_{1}}} a_{\ell_{1}}\left(\begin{array}{c}
\ell_{1} \\
2 \ell_{2}
\end{array}\right) \frac{4^{\ell_{2}}\left(1-(p-q)^{2}\right)^{\ell_{1}-2 \ell_{2}}}{(p+q+1)^{2 \ell_{1}-2 \ell_{2}-1}}+\mathcal{O}_{2 M}(p, q) \\
& \alpha^{-}(p, q)=2 \sum_{\substack{1 \leqslant \ell_{1} \leqslant 2 M \\
2 \ell_{2}+1 \leqslant \ell_{1}}} a_{\ell_{1}}\left(\begin{array}{c}
\ell_{1} \\
2 \ell_{2}+1
\end{array}\right) \frac{4^{\ell_{2}}\left(1-(p-q)^{2}\right)^{\ell_{1}-2 \ell_{2}-1}}{(p+q+1)^{2 \ell_{1}-2 \ell_{2}-2}}+\mathcal{O}_{2 M}(p, q) .
\end{aligned}
$$

In the above expansion, some $\mathcal{O}_{2 M}(p, q)$ terms are kept for notational simplicity. Now, one may check that for all functions $\theta$ and $\theta^{\prime}$ supported in $\left[r_{0}, \infty\left[{ }^{d} \times \mathbb{Z}^{d} \times \mathbb{R}\right.\right.$ and any integers $M_{1}$ and $M_{2}$, we have for all $j \in\{1, \ldots, d\}$,

$$
\left(f=\mathcal{O}_{M_{1}} \text { and } \theta \stackrel{M_{2}}{=} \theta^{\prime}\right) \Longrightarrow f\left(n_{j}, m_{j}\right) \theta(\widehat{w}) \stackrel{M_{1}+M_{2}}{\equiv} f\left(n_{j}, m_{j}\right) \theta^{\prime}(\widehat{w}) \text {. }
$$

Then Assertion (4.10) implies that for any function $g$ in $\mathcal{S}_{d}^{+}$supported in the set $\left[r_{0}, \infty\left[{ }^{d} \times \mathbb{Z}^{d} \times \mathbb{R}\right.\right.$, and any $j$ in $\{1, \ldots, d\}$, we have

$$
\begin{aligned}
& \alpha^{+}\left(n_{j}, m_{j}\right) \Theta_{g}(\widehat{w}) \\
& \stackrel{2 M-1}{\equiv}\left(n_{j}+m_{j}+1+\sum_{\substack{1 \leqslant \ell_{1} \leqslant 2 M \\
2 \ell_{2} \leqslant \ell_{1}}} a_{\ell_{1}}\left(\begin{array}{c}
\ell_{1} \\
2 \ell_{2}
\end{array}\right) \frac{4^{\ell_{2}}\left(1-\left(n_{j}-m_{j}\right)^{2}\right)^{\ell_{1}-2 \ell_{2}}}{\left(n_{j}+m_{j}+1\right)^{2 \ell_{1}-2 \ell_{2}-1}}\right) \Theta_{g}(\widehat{w})
\end{aligned}
$$

and

$$
\begin{aligned}
& \alpha^{-}\left(n_{j}, m_{j}\right) \theta_{g}(\widehat{w}) \\
& \stackrel{2 M-1}{\equiv} 2\left(\sum_{\substack{1 \leqslant \ell_{1} \leqslant 2 M \\
2 \ell_{2}+1 \leqslant \ell_{1}}} a_{\ell_{1}}\left(\begin{array}{c}
\ell_{1} \\
2 \ell_{2}+1
\end{array}\right) \frac{4^{\ell_{2}}\left(1-\left(n_{j}-m_{j}\right)^{2}\right)^{\ell_{1}-2 \ell_{2}-1}}{\left(n_{j}+m_{j}+1\right)^{2 \ell_{1}-2 \ell_{2}-2}}\right) \Theta_{g}(\widehat{w}) .
\end{aligned}
$$

Using (4.11), this gives

$$
\widetilde{\Delta}_{j}^{(0)}(\widehat{w}) \stackrel{2 M-1}{\equiv}\left(\frac{n_{j}+m_{j}+1}{2|\lambda|}\right) \Theta_{f}(\widehat{w})+\sum_{\ell=0}^{M} \Theta_{f_{j, 2 \ell}}(\widehat{w})
$$

with $f_{j, 0}(x, k, \lambda) \stackrel{\text { def }}{=} \sum_{\substack{1 \leqslant \ell_{1} \leqslant 2 M \\ 2 \ell_{2} \leqslant \ell_{1}}} a_{\ell_{1}}\left(\begin{array}{c}\ell_{1} \\ 2 \ell_{2}\end{array}\right) 2^{2 \ell_{2}-1}\left(1-k_{j}^{2}\right)^{\ell_{1}-2 \ell_{2}} \frac{\lambda^{2\left(\ell_{1}-\ell_{2}-1\right)}}{x_{j}^{2 \ell_{1}-2 \ell_{2}-1}} f(x, k, \lambda)$ 
and, if $1 \leqslant \ell \leqslant M$,

$$
f_{j, 2 \ell}(x, k, \lambda) \stackrel{\text { def }}{=} \frac{1}{(2 \ell) !} \sum_{\substack{0 \leqslant \ell_{1} \leqslant 2 M \\
2 \ell_{2} \leqslant \ell_{1}}} a_{\ell_{1}}\left(\begin{array}{c}
\ell_{1} \\
2 \ell_{2}
\end{array}\right) 2^{2 \ell_{2}-1+2 \ell}\left(1-k_{j}^{2}\right)^{\ell_{1}-2 \ell_{2}} \frac{\lambda^{2\left(\ell+\ell_{1}-\ell_{2}-1\right)}}{x_{j}^{2 \ell_{1}-2 \ell_{2}-1}} \partial_{x_{j}}^{2 \ell} f(x, k, \lambda) \text {. }
$$

Similarly,

$$
\widetilde{\Delta}_{j}^{(1)}(\widehat{w}) \stackrel{2 M-1}{\equiv} \sum_{\ell=0}^{M-1} \Theta_{f_{j, 2 \ell+1}}
$$

with $f_{j, 2 \ell+1}(x, k, \lambda) \stackrel{\text { def }}{=} \frac{1}{(2 \ell+1) !} \sum_{\substack{1 \leqslant \ell_{1} \leqslant 2 M \\ 2 \ell_{2}+1 \leqslant \ell_{1}}} a_{\ell_{1}}\left(\begin{array}{c}\ell_{1} \\ 2 \ell_{2}+1\end{array}\right) 4^{\ell_{2}+\ell}\left(1-k_{j}^{2}\right)^{\ell_{1}-2 \ell_{2}-1}$

$$
\times \frac{\lambda^{2\left(\ell+\ell_{1}-\ell_{2}-1\right)}}{x_{j}^{2 \ell_{1}-2 \ell_{2}-2}} \partial_{x_{j}}^{2 \ell+1} f(x, k, \lambda)
$$

From the definition of Operator $\widehat{\Delta}$, we thus infer that there exist functions $f_{\ell}$ of $\mathcal{S}_{d}^{+}$ supported in $\left[r_{0}, \infty\left[{ }^{d} \times \mathbb{Z}^{d} \times \mathbb{R}\right.\right.$ and satisfying (2.11), such that for all $M \geqslant 0$,

$$
\widehat{\Delta} \Theta_{f} \stackrel{2 M-1}{\equiv} \sum_{\ell=0}^{2 M} \Theta_{f_{\ell}}
$$

At this stage, one may prove by induction as in the previous case that $\left\|\widehat{\Delta}^{p} \Theta_{f}\right\|_{N, 0, \mathcal{S}\left(\widehat{\mathbb{H}}^{d}\right)}$ is finite for all integers $N$ and $p$.

Let us finally study the action of $\widehat{\mathcal{D}}_{\lambda}$. From its definition, setting $k=m-n$, we get

$$
\begin{aligned}
& \left(\widehat{\mathcal{D}}_{\lambda} \Theta_{f}\right)(\widehat{w})=\frac{\mathrm{d}}{\mathrm{d} \lambda}(f(|\lambda| R(n, m), k, \lambda))+\frac{d}{2 \lambda} f(|\lambda| R(n, m), k, \lambda)+\frac{1}{2 \lambda} \sum_{j=1}^{d} \mathcal{D}_{j}(\widehat{w}) \\
& \text { with } \quad \mathcal{D}_{j}(\widehat{w}) \stackrel{\text { def }}{=} \sqrt{n_{j} m_{j}} f\left(|\lambda|\left(R(n, m)-2 \delta_{j}\right), k, \lambda\right) \\
& -\sqrt{\left(n_{j}+1\right)\left(m_{j}+1\right)} f\left(|\lambda|\left(R(n, m)+2 \delta_{j}\right), k, \lambda\right) \text {. }
\end{aligned}
$$

The chain rule implies that

$$
\begin{aligned}
\frac{\mathrm{d}}{\mathrm{d} \lambda}(f(|\lambda| R(n, m), k, \lambda))=\left(\partial_{\lambda} f\right) & (|\lambda| R(n, m), k, \lambda) \\
& +\operatorname{sgn} \lambda \sum_{j=1}^{d}\left(n_{j}+m_{j}+1\right)\left(\partial_{x_{j}} f\right)(|\lambda| R(n, m), k, \lambda) .
\end{aligned}
$$

Combining Lemma 4.2, and Assertions (4.3) and (4.4) yields

$$
\begin{aligned}
-\frac{1}{2 \lambda} \mathcal{D}_{j}(\widehat{w}) \stackrel{2 M-1}{\equiv} \alpha^{-}\left(n_{j}, m_{j}\right) \sum_{\ell=0}^{M} \frac{(2 \lambda)^{2 \ell-1}}{(2 \ell) !}\left(\partial_{x_{j}}^{2 \ell} f\right)(|\lambda| R(n, m), k, \lambda) \\
+\alpha^{+}\left(n_{j}, m_{j}\right) \operatorname{sgn} \lambda \sum_{\ell=0}^{M-1} \frac{(2 \lambda)^{2 \ell}}{(2 \ell+1) !}\left(\partial_{x_{j}}^{2 \ell+1} f\right)(|\lambda| R(n, m), k, \lambda) .
\end{aligned}
$$


Therefore, we have

$$
\begin{aligned}
\left(\widehat{\mathcal{D}}_{\lambda} \Theta_{f}\right)(\widehat{w}) \stackrel{2 M-1}{\equiv}\left(\partial_{\lambda} f\right)(|\lambda| R(n, m), k, \lambda)+\frac{1}{2 \lambda}\left(d-\sum_{j=1}^{d} \alpha^{-}\left(n_{j}, m_{j}\right)\right) f(|\lambda| R(n, m), k, \lambda) \\
+\operatorname{sgn} \lambda \sum_{j=1}^{d}\left(n_{j}+m_{j}+1-\alpha^{+}\left(n_{j}, m_{j}\right)\right)\left(\partial_{x_{j}} f\right)(|\lambda| R(n, m), k, \lambda) \\
-\alpha^{-}\left(n_{j}, m_{j}\right) \sum_{\ell=1}^{M} \frac{(2 \lambda)^{2 \ell-1}}{(2 \ell) !}\left(\partial_{x_{j}}^{2 \ell} f\right)(|\lambda| R(n, m), k, \lambda) \\
-\alpha^{+}\left(n_{j}, m_{j}\right) \operatorname{sgn} \lambda \sum_{\ell=1}^{M-1} \frac{(2 \lambda)^{2 \ell}}{(2 \ell+1) !}\left(\partial_{x_{j}}^{2 \ell+1} f\right)(|\lambda| R(n, m), k, \lambda) .
\end{aligned}
$$

Hence, using (4.12) and (4.13) and noticing that the coefficient $a_{\ell_{1}}$ involved in the expansion of $\alpha^{ \pm}\left(n_{j}, m_{j}\right)$ is equal to $1 / 2$, we conclude that there exist some functions $\tilde{f}_{j}, f_{j}^{b}$ and $f_{j, \ell}^{\sharp}$ of $\mathcal{S}_{d}^{+}$, supported in $\left[r_{0}, \infty\left[^{d} \times \mathbb{Z}^{d} \times \mathbb{R}\right.\right.$ and satisfying (2.11) so that

$$
\left(\widehat{\mathcal{D}}_{\lambda} \Theta_{f}\right)(\widehat{w})-\left(\Theta_{\partial_{\lambda} f}\right)(\widehat{w}) \stackrel{2 M-1}{=} \sum_{j=1}^{d} \Theta_{\widetilde{f}_{j}}(\widehat{w})-\sum_{j=1}^{d} \Theta_{f_{j}^{\natural}}(\widehat{w})-\sum_{\ell=1}^{2 M} \sum_{j=1}^{d} \Theta_{f_{j, \ell}^{\sharp}}(\widehat{w}),
$$

where

$$
\begin{aligned}
\tilde{f}_{j}(x, k, \lambda) \stackrel{\text { def }}{=} \sum_{\substack{2 \leqslant \ell_{1} \leqslant 2 M \\
2 \ell_{2}+1 \leqslant \ell_{1}}} a_{\ell_{1}}\left(\begin{array}{c}
\ell_{1} \\
2 \ell_{2}+1
\end{array}\right) \frac{4^{\ell_{2}}\left(1-k_{j}^{2}\right)^{\ell_{1}-2 \ell_{2}-1} \lambda^{2 \ell_{1}-2 \ell_{2}-3}}{x_{j}^{2 \ell_{1}-2 \ell_{2}-2}} f(x, k, \lambda), \\
f_{j}^{b}(x, k, \lambda) \stackrel{\text { def }}{=} \sum_{\substack{1 \leqslant \ell_{1} \leqslant 2 M \\
2 \ell_{2} \leqslant \ell_{1}}} a_{\ell_{1}}\left(\begin{array}{c}
\ell_{1} \\
2 \ell_{2}
\end{array}\right) \frac{4^{\ell_{2}}\left(1-k_{j}^{2}\right)^{\ell_{1}-2 \ell_{2}} \lambda^{2 \ell_{1}-2 \ell_{2}-1}}{x_{j}^{2 \ell_{1}-2 \ell_{2}-1}} \partial_{x_{j}} f(x, k, \lambda), \\
f_{j, 2 \ell}^{\sharp}(x, k, \lambda) \stackrel{\text { def }}{=}\left(\sum_{\substack{1 \leqslant \ell_{1} \leqslant 2 M \\
2 \ell_{2}+1 \leqslant \ell_{1}}} a_{\ell_{1}}\left(\begin{array}{c}
\ell_{1} \\
2 \ell_{2}+1
\end{array}\right) \frac{2^{2 \ell_{2}+2 \ell}\left(1-k_{j}^{2}\right)^{\ell_{1}-2 \ell_{2}-1}}{x_{j}^{2 \ell_{1}-2 \ell_{2}-2}}\right) \frac{\lambda^{2 \ell+2 \ell_{1}-2 \ell_{2}-3}}{(2 \ell) !}\left(\partial_{x_{j}}^{2 \ell} f\right)(x, k, \lambda)
\end{aligned}
$$

and

$$
\begin{array}{r}
f_{j, 2 \ell+1}^{\sharp}(x, k, \lambda) \stackrel{\text { def }}{=} 2\left(x_{j}+\sum_{\substack{1 \leqslant \ell_{1} \leqslant 2 M \\
2 \ell_{2} \leqslant \ell_{1}}} a_{\ell_{1}}\left(\begin{array}{c}
\ell_{1} \\
2 \ell_{2}
\end{array}\right) \frac{4^{\ell_{2}}\left(1-k_{j}^{2}\right)^{\ell_{1}-2 \ell_{2}} \lambda^{2 \ell_{1}-2 \ell_{2}}}{x_{j}^{2 \ell_{1}-2 \ell_{2}-1}}\right) \\
\times \frac{(2 \lambda)^{2 \ell-1}}{(2 \ell+1) !}\left(\partial_{x_{j}}^{2 \ell+1} f\right)(x, k, \lambda) .
\end{array}
$$

At this stage, one can complete the proof as in the previous cases.

Let us finally record two interesting asymptotic properties of the operators $\widehat{\Delta}$ and $\widehat{\mathcal{D}}_{\lambda}$ when $\lambda$ tends to 0 . 
Proposition 4.3. - For any function $f$ in $\mathcal{S}_{1}^{+}$supported in $\left[r_{0}, \infty[\times \mathbb{Z} \times \mathbb{R}\right.$ for some positive $r_{0}$, the extension of $\widehat{\Delta} \Theta_{f}$ and $\widehat{\mathcal{D}}_{\lambda} \Theta_{f}$ to $\widehat{\mathbb{H}}_{0}^{1}$ is given by

$$
\begin{aligned}
\left(\widehat{\Delta}_{\Theta_{f}}\right)(\dot{x}, k) & =\dot{x} \partial_{\dot{x} \dot{x}}^{2} f(\dot{x}, k, 0)+\partial_{\dot{x}} f(\dot{x}, k, 0)-\frac{k^{2}}{4 \dot{x}} f(\dot{x}, k, 0) \\
\text { and } \quad\left(\widehat{\mathcal{D}}_{\lambda} \Theta_{f}\right)(\dot{x}, k) & =\partial_{\lambda} f(\dot{x}, k, 0) .
\end{aligned}
$$

Proof. - For expository purpose, we omit the dependency on $k$, for $f$. Then we have by definition of $\Theta_{f}$ and $\widehat{\Delta}$, for all $(n, n+k, \lambda)$ in $\widetilde{\mathbb{H}}^{1}$ with positive $\lambda$,

$$
\begin{aligned}
-2 \lambda^{2} \widehat{\Delta} \Theta_{f}(n, n+k, \lambda)=\lambda & (2 n+k+1) f(\lambda(2 n+k+1), \lambda) \\
& -\lambda \sqrt{(n+1)(n+k+1)} f(\lambda(2 n+k+3), \lambda) \\
& -\lambda \sqrt{n(n+k)} f(\lambda(2 n+k-1), \lambda) .
\end{aligned}
$$

Denoting $\dot{x}=2 \lambda n$, the above equality rewrites

$$
\begin{array}{ll}
-2 \lambda^{2} \widehat{\Delta} \Theta_{f}(\widehat{w}) & =\widetilde{\Delta}^{1}(\widehat{w})-\widetilde{\Delta}^{2}(\widehat{w})-\widetilde{\Delta}^{3}(\widehat{w}) \\
\text { with } \quad \widetilde{\Delta}^{1}(\widehat{w}) & \stackrel{\text { def }}{=}(\dot{x}+\lambda(k+1)) f(\dot{x}+\lambda(k+1), \lambda), \\
& \widetilde{\Delta}^{2}(\widehat{w}) \stackrel{\text { def }}{=} \sqrt{\left(\frac{\dot{x}}{2}+\lambda\right)\left(\frac{\dot{x}}{2}+\lambda(k+1)\right)} f(\dot{x}+\lambda(k+3), \lambda), \\
\widetilde{\Delta}^{3}(\widehat{w}) \stackrel{\text { def }}{=} \sqrt{\frac{\dot{x}}{2}\left(\frac{\dot{x}}{2}+\lambda k\right)} f(\dot{x}+\lambda(k-1), \lambda) .
\end{array}
$$

Let us compute the second order expansions of $\widetilde{\Delta}^{1}(\widehat{w}), \widetilde{\Delta}^{2}(\widehat{w})$ and $\widetilde{\Delta}^{3}(\widehat{w})$ with respect to $\lambda$, for fixed (and positive) value of $\dot{x}=2 \lambda n$. A Taylor expansion of $f$ near the point $(\dot{x}, 0)$ gives

$$
\begin{aligned}
\widetilde{\Delta}^{1}(\widehat{w})= & \dot{x} f(\dot{x}, 0)+\left((k+1)\left(f(\dot{x}, 0)+\dot{x} \partial_{\dot{x}} f(\dot{x}, 0)\right)+\dot{x} \partial_{\lambda} f(\dot{x}, 0)\right) \lambda \\
+\left(\frac{1}{2} \dot{x} \partial_{\lambda \lambda}^{2} f(\dot{x}, 0)\right. & +\frac{(k+1)^{2}}{2}\left(\dot{x} \partial_{\dot{x} \dot{x}}^{2} f(\dot{x}, 0)+2 \partial_{\dot{x}} f(\dot{x}, 0)\right) \\
& \left.+(k+1)\left(\partial_{\lambda} f(\dot{x}, 0)+\dot{x} \partial_{\dot{x} \lambda}^{2} f(\dot{x}, 0)\right)\right) \lambda^{2}+\mathcal{O}\left(\lambda^{3}\right)
\end{aligned}
$$

where as in all that follows, $\mathcal{O}\left(\lambda^{N}\right)$ denotes any function of $(\dot{x}, k, \lambda)$ such that there exists a constant $C_{r_{0}, k}$ depending only on $f, r_{0}$ and $k$ such that

$$
\left|\mathcal{O}\left(\lambda^{N}\right)\right| \leqslant C_{r_{0}, k}|\lambda|^{N}
$$

In order to expand the terms $\widetilde{\Delta}^{2}(\widehat{w})$ and $\widetilde{\Delta}^{3}(\widehat{w})$, we shall use the fact that there exists some constant $C$ such that for all $\dot{y}>0$ and $\eta$ in $]-\dot{y}, \dot{y}[$, we have

$$
\left|\sqrt{\dot{y}+\eta}-\sqrt{\dot{y}}-\frac{\eta}{2 \sqrt{\dot{y}}}+\frac{\eta^{2}}{8 \dot{y} \sqrt{\dot{y}}}\right| \leqslant C \sqrt{\dot{y}}\left|\frac{\eta}{\dot{y}}\right|^{3} \text {. }
$$


In order to find out the second order expansion of $\widetilde{\Delta}^{2}$, let us use (4.16) with $\dot{y}=\dot{x} / 2$ and $\eta=\lambda$ or $\eta=\lambda(k+1)$ together with the Taylor expansion of $f$ at $(\dot{x}, 0)$. This gives

$$
\begin{aligned}
\widetilde{\Delta}^{2}(\widehat{w})= & \left(\sqrt{\dot{y}}+\frac{\lambda}{2 \sqrt{\dot{y}}}-\frac{\lambda^{2}}{8 \dot{y} \sqrt{\dot{y}}}\right)\left(\sqrt{\dot{y}}+\frac{(k+1) \lambda}{2 \sqrt{\dot{y}}}-\frac{(k+1)^{2} \lambda^{2}}{8 \dot{y} \sqrt{\dot{y}}}\right) \\
& \times\left(f(\dot{x}, 0)+\left(\partial_{\lambda} f(\dot{x}, 0)+(k+3) \partial_{\dot{x}} f(\dot{x}, 0)\right) \lambda\right. \\
& \left.+\left(\frac{1}{2} \partial_{\lambda \lambda}^{2} f(\dot{x}, 0)+(k+3) \partial_{\dot{x} \lambda}^{2} f(\dot{x}, 0)+\frac{(k+3)^{2}}{2} \partial_{\dot{x} \dot{x}}^{2} f(\dot{x}, 0)\right) \lambda^{2}\right)+\mathcal{O}\left(\lambda^{3}\right) .
\end{aligned}
$$

Hence, we get in the end, replacing $\dot{y}$ by its value,

$$
\begin{aligned}
\widetilde{\Delta}^{2}(\widehat{w}) & =\frac{\dot{x}}{2} f(\dot{x}, 0)+\left(\left(1+\frac{k}{2}\right) f(\dot{x}, 0)+\left(\frac{k+3}{2}\right) \dot{x} \partial_{\dot{x}} f(\dot{x}, 0)+\frac{1}{2} \dot{x} \partial_{\lambda} f(\dot{x}, 0)\right) \lambda \\
+ & \left(\frac{(k+3)^{2}}{4} \dot{x} \partial_{\dot{x} \dot{x}}^{2} f(\dot{x}, 0)+\left(\frac{k+3}{2}\right) \dot{x} \partial_{\dot{x} \lambda}^{2} f(\dot{x}, 0)+\frac{\dot{x}}{4} \partial_{\lambda \lambda}^{2} f(\dot{x}, 0)\right. \\
& \left.+\left(1+\frac{k}{2}\right)\left((k+3) \partial_{\dot{x}} f(\dot{x}, 0)+\partial_{\lambda} f(\dot{x}, 0)\right)-\frac{k^{2}}{4 \dot{x}} f(\dot{x}, 0)\right) \lambda^{2}+\mathcal{O}\left(\lambda^{3}\right) .
\end{aligned}
$$

Similarly, we have

$$
\begin{aligned}
\widetilde{\Delta}^{3}(\widehat{w})= & \sqrt{\dot{y}}\left(\sqrt{\dot{y}}+\frac{k \lambda}{2 \sqrt{\dot{y}}}-\frac{k^{2} \lambda^{2}}{8 \dot{y} \sqrt{\dot{y}}}\right)\left(f(\dot{x}, 0)+\left(\partial_{\lambda} f(\dot{x}, 0)+(k-1) \partial_{\dot{x}} f(\dot{x}, 0)\right) \lambda\right. \\
& \left.+\left(\frac{1}{2} \partial_{\lambda \lambda}^{2} f(\dot{x}, 0)+(k-1) \partial_{\dot{x} \lambda}^{2} f(\dot{x}, 0)+\frac{(k-1)^{2}}{2} \partial_{\dot{x} \dot{x}}^{2} f(\dot{x}, 0)\right) \lambda^{2}\right)+\mathcal{O}\left(\lambda^{3}\right),
\end{aligned}
$$

whence,

$$
\begin{aligned}
\widetilde{\Delta}^{3}(\widehat{w})= & \frac{\dot{x}}{2} f(\dot{x}, 0)+\left(\frac{k}{2} f(\dot{x}, 0)+\left(\frac{k-1}{2}\right) \dot{x} \partial_{\dot{x}} f(\dot{x}, 0)+\frac{1}{2} \dot{x} \partial_{\lambda} f(\dot{x}, 0)\right) \lambda \\
+ & \left(\frac{(k-1)^{2}}{4} \dot{x} \partial_{\dot{x} \dot{x}}^{2} f+\left(\frac{k-1}{2}\right) \dot{x} \partial_{\dot{x} \lambda}^{2} f(\dot{x}, 0)+\frac{\dot{x}}{4} \partial_{\lambda \lambda}^{2} f(\dot{x}, 0)\right. \\
& \left.+\frac{k}{2}\left((k-1) \partial_{\dot{x}} f(\dot{x}, 0)+\partial_{\lambda} f(\dot{x}, 0)\right)-\frac{k^{2}}{4 \dot{x}} f(\dot{x}, 0)\right) \lambda^{2}+\mathcal{O}\left(\lambda^{3}\right) .
\end{aligned}
$$

Inserting inequalities (4.15), (4.17) and (4.18) in (4.14), we discover that the zeroth and first order terms in the expansion cancel. Hence we are left with

$$
\left|2 \widehat{\Delta} \Theta_{f}(\widehat{w})+\frac{k^{2}}{2 \dot{x}} f(\dot{x}, 0)-2 \partial_{\dot{x}} f(\dot{x}, 0)-2 \dot{x} \partial_{\dot{x} \dot{x}}^{2} f(\dot{x}, 0)\right| \leqslant C_{r_{0}, k}|\lambda| .
$$

Now let us consider a sequence $\left(n_{p}, \lambda_{p}\right)_{p \in \mathbb{N}}$ such that $2 \lim _{p \rightarrow \infty} \lambda_{p} n_{p}=\dot{x}_{0}$ and $\lim _{p \rightarrow \infty} \lambda_{p}=0$. Applying the above inequality (4.19) with $\dot{x}=2 \lambda_{p} n_{p}$ and $\lambda=\lambda_{p}$ 
ensures that

$$
\begin{aligned}
\lim _{p \rightarrow \infty}\left(\widehat{\Delta} \Theta_{f}\left(n_{p}, n_{p}+k, \lambda_{p}\right)+\frac{k^{2}}{8 \lambda_{p} n_{p}}\right. & f\left(2 \lambda_{p} n_{p}, 0\right) \\
& \left.-\partial_{\dot{x}} f\left(2 \lambda_{p} n_{p}, 0\right)-2 \lambda_{p} n_{p} \partial_{\dot{x} \dot{x}}^{2} f\left(2 \lambda_{p} n_{p}, 0\right)\right)=0 .
\end{aligned}
$$

As the function $f$ is regular with respect to $\dot{x}$ on $\left[r_{0}, \infty[\right.$, we infer that

$$
\lim _{p \rightarrow \infty} \widehat{\Delta} \Theta_{f}\left(n_{p}, n_{p}+k, \lambda_{p}\right)=\dot{x}_{0} \partial_{\dot{x} \dot{x}}^{2} f\left(\dot{x}_{0}, 0\right)+\partial_{\dot{x}} f\left(\dot{x}_{0}, 0\right)-\frac{k^{2}}{4 \dot{x}} f\left(\dot{x}_{0}, 0\right) .
$$

The proof for Operator $\widehat{\mathcal{D}}_{\lambda}$ being quite similar, we just sketch it. From the definition of $\widehat{\mathcal{D}}_{\lambda}$ and the chain rule, we discover that for all $(n, n+k, \lambda)$ in $\widetilde{\mathbb{H}}^{1}$ with $\lambda>0$,

$$
\begin{aligned}
\widehat{\mathcal{D}}_{\lambda} \Theta_{f}(\widehat{w})= & (2 n+k+1) \partial_{\dot{x}} f(\lambda(2 n+k+1), \lambda)+\partial_{\lambda} f(\lambda(2 n+k+1), \lambda) \\
& +\frac{1}{2 \lambda}(f(\lambda(2 n+k+1), \lambda)+\sqrt{n(n+k)} f(\lambda(2 n+k-1), \lambda) \\
& -\sqrt{(n+1)(n+k+1)} f(\lambda(2 n+k+3), \lambda)) .
\end{aligned}
$$

Therefore, assuming that $\dot{x} \stackrel{\text { def }}{=} 2 \lambda n>0$, we get

$$
\begin{array}{r}
\widehat{\mathcal{D}}_{\lambda} \Theta_{f}(\widehat{w})=\partial_{\lambda} f(\dot{x}+(k+1) \lambda, \lambda)+\frac{1}{\lambda}(\dot{x}+(k+1) \lambda) \partial_{\dot{x}} f(\dot{x}+(k+1) \lambda, \lambda) \\
+\frac{1}{2 \lambda^{2}}\left(\lambda f(\dot{x}+(k+1) \lambda, \lambda)+\widetilde{\Delta}^{3}(\widehat{w})-\widetilde{\Delta}^{2}(\widehat{w})\right) .
\end{array}
$$

Because

$$
f(\dot{x}+(k+1) \lambda, \lambda)=f(\dot{x}, 0)+\left((k+1) \partial_{\dot{x}} f(\dot{x}, 0)+\partial_{\lambda} f(\dot{x}, 0)\right) \lambda+\mathcal{O}\left(\lambda^{2}\right)
$$

and

$$
\begin{aligned}
(\dot{x}+ & (k+1) \lambda) \partial_{\dot{x}} f(\dot{x}+(k+1) \lambda, \lambda) \\
& =\dot{x} \partial_{\dot{x}} f(\dot{x}, 0)+\left((k+1)\left(\partial_{\dot{x}} f(\dot{x}, 0)+\dot{x} \partial_{\dot{x} \dot{x}}^{2} f(\dot{x}, 0)\right)+\dot{x} \partial_{\dot{x} \lambda}^{2} f(\dot{x}, 0)\right) \lambda+\mathcal{O}\left(\lambda^{2}\right),
\end{aligned}
$$

we get in the end, taking advantage of (4.17) and (4.18),

$$
\widehat{\mathcal{D}}_{\lambda} \Theta_{f}(\widehat{w})=\partial_{\lambda} f(\dot{x}, 0)+\mathcal{O}(\lambda)
$$

which completes the proof.

\section{Examples of tempered distributions}

A first class of examples is given by the functions belonging to the space $L_{M}^{1}\left(\widehat{\mathbb{H}}^{d}\right)$ of Definition 2.11. This is exactly what states Theorem 2.12 that we are going to prove now. 
Proof of Theorem 2.12. - Inequality (2.13) just follows from the definition of the semi-norms on $\widehat{\mathbb{H}}^{d}$. So let us focus on the proof of the first part of the statement that actually holds true in the largest class of locally integrable functions.

Let $f$ be a function of $L_{l o c}^{1}\left(\widehat{\mathbb{H}}^{d}\right)$ such that $\iota(f)=0$. In order to prove that $f=0$ a.e., it suffices to check that for all $K>0$ and $b>a>0$, we have

$$
\int_{\widehat{\mathcal{C}}_{a, b, K}}|f(\widehat{w})| \mathrm{d} \widehat{w}=0
$$

with $\widehat{\mathcal{C}}_{a, b, K} \stackrel{\text { def }}{=}\left\{(n, m, \lambda) \in \widehat{\mathbb{H}}^{d}:|\lambda|(|n+m|+d) \leqslant K,|n-m| \leqslant K\right.$ and $\left.a \leqslant|\lambda| \leqslant b\right\}$.

To this end, we introduce the bounded function :

$$
g \stackrel{\text { def }}{=} \frac{\bar{f}}{|f|} \mathbb{1}_{f \neq 0} \mathbb{1}_{\widehat{\mathcal{C}}_{a, b, K}}
$$

and smooth it out with respect to $\lambda$ by setting

$$
g_{\varepsilon} \stackrel{\text { def }}{=} \chi_{\varepsilon} \star_{\lambda} g
$$

where $\chi_{\varepsilon} \stackrel{\text { def }}{=} \varepsilon^{-1} \chi\left(\varepsilon^{-1}\right.$.) and $\chi$ stands for some smooth even function on $\mathbb{R}$, supported in the interval $[-1,1]$ and with integral 1 .

Note that by definition, $g$ is supported in the set $\widehat{\mathcal{C}}_{a, b, K}$. Therefore, if $\varepsilon<a$ then $g_{\varepsilon}$ is supported in $\widehat{\mathcal{C}}_{a-\varepsilon, b+\varepsilon, K(1+\varepsilon / a)}$. This readily ensures that $\left\|g_{\varepsilon}\right\|_{N, 0, \mathcal{S}\left(\widehat{\mathbb{H}}^{d}\right)}$ is finite for all integer $N$ (as regards the action of operator $\widehat{\Sigma}_{0}$, note that $g_{\varepsilon}(n, m, \lambda)=0$ whenever $|\lambda|<a-\varepsilon)$.

In order to prove that $g_{\varepsilon}$ belongs to $\mathcal{S}\left(\widehat{\mathbb{H}}^{d}\right)$, it suffices to use the following lemma the proof of which is left to the reader:

Lemma 5.1. - Let $h$ be a smooth function on $\widehat{\mathbb{H}}^{d}$ with support in $\{(n, m, \lambda)$ : $|\lambda| \geqslant a\}$ for some $a>0$. If $h$ and all derivatives with respect to $\lambda$ have fast decay, that is have finite semi-norm $\|\cdot\|_{N, 0, \mathcal{S}\left(\widehat{\mathbb{H}}^{d}\right)}$ for all integer $N$, then the same properties hold true for $\widehat{\mathcal{D}}_{\lambda} h$ and $\widehat{\Delta} h$.

Because $g_{\varepsilon}$ is in $\mathcal{S}\left(\widehat{\mathbb{H}}^{d}\right)$ for all $0<\varepsilon<a$, our assumption on $f$ ensures that we have

$$
I_{\varepsilon} \stackrel{\text { def }}{=} \int_{\widehat{\mathbb{H}^{d}}} f(\widehat{w}) g_{\varepsilon}(\widehat{w}) \mathrm{d} \widehat{w}=0 .
$$

Let $\widetilde{K}=3 K / 2$. Whenever $0<\varepsilon \leqslant a / 2$, we have for all $(n, m, \lambda) \in \widetilde{\mathbb{H}}^{d}$ and $\lambda^{\prime} \neq 0$,

$$
\begin{aligned}
\frac{1}{\varepsilon} \chi\left(\frac{\lambda-\lambda^{\prime}}{\varepsilon}\right) g\left(n, m, \lambda^{\prime}\right) f(n, & m, \lambda) \\
& =\frac{1}{\varepsilon} \chi\left(\frac{\lambda-\lambda^{\prime}}{\varepsilon}\right) g\left(n, m, \lambda^{\prime}\right)\left(\mathbb{1}_{\widehat{\mathcal{C}}_{a / 2, b+a / 2, \widetilde{K}}} f\right)(n, m, \lambda),
\end{aligned}
$$

which guarantees that

$$
\int_{\widehat{\mathbb{H}} d \times \mathbb{R}} \chi_{\varepsilon}\left(\lambda-\lambda^{\prime}\right)\left|g\left(n, m, \lambda^{\prime}\right)\right||f(n, m, \lambda)| \mathrm{d} \widehat{w} \mathrm{~d} \lambda^{\prime} \leqslant\|\chi\|_{L^{1}}\left\|\mathbb{1}_{\widehat{\mathcal{C}}_{a / 2, b+a / 2, \widetilde{K}}} f\right\|_{L^{1}}<\infty .
$$


Therefore applying Fubini theorem, remembering that $\chi$ is an even function and exchanging the notation $\lambda$ and $\lambda^{\prime}$ in the second line below,

$$
\begin{aligned}
I_{\varepsilon} & =\int_{\widehat{\mathbb{H}}^{d}} f(n, m, \lambda)\left(\int_{\mathbb{R}} \chi_{\varepsilon}\left(\lambda-\lambda^{\prime}\right) g\left(n, m, \lambda^{\prime}\right) \mathrm{d} \lambda^{\prime}\right) \mathrm{d} \widehat{w} \\
& =\int_{\widehat{\mathbb{H}}^{d}} g(n, m, \lambda)\left(\int_{\mathbb{R}} \chi_{\varepsilon}\left(\lambda-\lambda^{\prime}\right)\left(1_{\widehat{\mathcal{C}^{a / 2, b+a / 2, \widetilde{K}}}} f\right)\left(n, m, \lambda^{\prime}\right) \mathrm{d} \lambda^{\prime}\right) \mathrm{d} \widehat{w} \\
& =\int_{\widehat{\mathbb{H} d}}\left(\chi_{\varepsilon} \star\left(\mathbb{1}_{\widehat{\mathcal{C}}}{ }_{a / 2, b+a / 2, \widetilde{K}} f\right)\right)(\widehat{w}) g(\widehat{w}) \mathrm{d} \widehat{w} .
\end{aligned}
$$

The standard density theorem for convolution in $\mathbb{R}$ ensures that for all $(n, m)$ in $\mathbb{N}^{2 d}$, we have

$$
\lim _{\varepsilon \rightarrow 0} \int_{\mathbb{R}}\left|\chi_{\varepsilon} \star\left(\mathbb{1}_{\widehat{\mathcal{C}}_{a / 2, b+a / 2, \widetilde{K}}} f\right)(n, m, \lambda)-\left(\mathbb{1}_{\widehat{\mathcal{C}}_{a / 2, b+a / 2, \widetilde{K}}} f\right)(n, m, \lambda)\right| \mathrm{d} \lambda=0 .
$$

Hence, because the supremum of $g$ is bounded by 1 , we get

$$
\begin{aligned}
0 & =\lim _{\varepsilon \rightarrow 0} I_{\varepsilon} \\
& =\int_{\widehat{\mathbb{H}} d} \mathbb{1}_{\widehat{\mathcal{C}_{a / 2, b+a / 2, \widetilde{K}}}} f(\widehat{w}) g(\widehat{w}) \mathrm{d} \widehat{w} \\
& =\int_{\widehat{\mathcal{C}}}|f(\widehat{w})| \mathrm{d} \widehat{w},
\end{aligned}
$$

which completes the proof of Theorem 2.12.

Proof of Proposition 2.13. - It claims that the functions

$$
f_{\gamma}(n, m, \lambda) \stackrel{\text { def }}{=}(|\lambda|(2|m|+d))^{-\gamma} \delta_{n, m}
$$

are in $L_{M}^{1}$ in the case when $\gamma$ is less than $d+1$. As $f_{\gamma}$ is continuous and bounded away from any neighborhood of $\widehat{0}$, it suffices to prove that

$$
\sum_{n \in \mathbb{N}^{d}} \int_{|\lambda|(2|n|+d) \leqslant 1} f_{\gamma}(n, n, \lambda)|\lambda|^{d} \mathrm{~d} \lambda<\infty .
$$

Now, performing the change of variables $\lambda^{\prime}=\lambda(2|n|+d)$, we discover that, since $\gamma<d+1$,

$$
\left.\sum_{n \in \mathbb{N}^{d}} \int_{|\lambda|(2|n|+d) \leqslant 1} f_{\gamma}(n, n, \lambda) \lambda\right|^{d} \mathrm{~d} \lambda=\sum_{n \in \mathbb{N}^{d}}(2|n|+d)^{-d-1} \int_{\left|\lambda^{\prime}\right| \leqslant 1}\left|\lambda^{\prime}\right|^{d-\gamma} \mathrm{d} \lambda^{\prime}<\infty .
$$

Hence one may conclude that $f_{\gamma}$ is in $L_{M}^{1}$.

Proof of Proposition 2.15. - We start with the obvious observation that

$$
\left|\int_{\widehat{\mathbb{H}}^{d}}\left(\frac{\theta(n, n, \lambda)+\theta(n, n,-\lambda)-2 \theta(\widehat{0})}{|\lambda|^{\gamma}(2|n|+d)^{\gamma}}\right) \delta_{n, m} \mathrm{~d} \widehat{w}\right| \leqslant \mathcal{I}_{1}+\mathcal{I}_{2}
$$

with

$$
\begin{aligned}
& \mathcal{I}_{1} \stackrel{\text { def }}{=} \int_{\widehat{\mathbb{H}} d} \mathbb{1}_{\{|\lambda|(2|n|+d) \geqslant 1\}} \delta_{n, m} \frac{|\theta(n, n, \lambda)+\theta(n, n,-\lambda)-2 \theta(\widehat{0})|}{|\lambda|^{\gamma}(2|n|+d)^{\gamma}} \mathrm{d} \widehat{w} \\
& \text { and } \quad \mathcal{I}_{2} \stackrel{\text { def }}{=} \int_{\widehat{\mathbb{H}} d} \mathbb{1}_{\{|\lambda|(2|n|+d)<1\}} \delta_{n, m} \frac{|\theta(n, n, \lambda)+\theta(n, n,-\lambda)-2 \theta(\widehat{0})|}{|\lambda|^{\gamma}(2|n|+d)^{\gamma}} \mathrm{d} \widehat{w} .
\end{aligned}
$$


On the one hand, we have, changing variable $\lambda^{\prime}=\lambda(2|n|+d)$ and using that $\gamma>d+1$,

$$
\mathcal{I}_{1} \leqslant 4\|\theta\|_{L^{\infty}\left(\widehat{\mathbb{H}}^{d}\right)} \sum_{n \in \mathbb{N}^{d}} \frac{2}{(2|n|+d)^{d+1}} \int_{1}^{\infty}\left|\lambda^{\prime}\right|^{d-\gamma} \mathrm{d} \lambda^{\prime} \leqslant C\|\theta\|_{L^{\infty}\left(\widehat{\mathbb{H}}^{d}\right)} .
$$

On the other hand, changing again variable $\lambda^{\prime}=\lambda \mid(2|n|+d)$, we see that

$$
\begin{aligned}
& \mathcal{I}_{2}=\sum_{n \in \mathbb{N}^{d}} \frac{2}{(2|n|+d)^{d+1}} \\
& \quad \times \int_{0}^{1}\left(\theta\left(n, n, \frac{\lambda^{\prime}}{2|n|+d}\right)+\theta\left(n, n, \frac{-\lambda^{\prime}}{2|n|+d}\right)-2 \theta(\widehat{0})\right)\left|\lambda^{\prime}\right|^{d-\gamma} \mathrm{d} \lambda^{\prime} .
\end{aligned}
$$

At this stage, we need a suitable bound of the integrand just above. This will be achieved thanks to the following lemma.

LemmA 5.2. - There exists an integer $k$ such that for any function $\theta$ in $\mathcal{S}\left(\widehat{\mathbb{H}}^{d}\right)$, we have

$$
\forall(n, m, \lambda) \in \widehat{\mathbb{H}}^{d},\left|\theta(n, m, \lambda)-\theta(\widehat{0}) \delta_{n, m}\right| \leqslant C\|\theta\|_{k, k, \mathcal{S}\left(\widehat{\mathbb{H}}^{d}\right)}(\sqrt{|\lambda|(2|n|+d)}+|\lambda|) .
$$

Proof. - Theorem 2.6 guarantees that $\theta$ is the Fourier transform of a function $f$ of $\mathcal{S}\left(\mathbb{H}^{d}\right)$ (with control of semi-norms). Hence it suffices to prove that

$$
\begin{aligned}
&\left|\widehat{f}_{\mathbb{H}}(\widehat{w})-\delta_{n, m} \int_{\mathbb{H}^{d}} f(w) \mathrm{d} w\right| \leqslant C N(f)\left(\sqrt{|\lambda|(|n+m|+d)}+|\lambda| \delta_{n, m}\right) \\
& \text { with } \quad N(f) \stackrel{\text { def }}{=} \int_{\mathbb{H}^{d}}(1+|Y|+|s+2\langle\eta, y\rangle|)|f(Y, s)| \mathrm{d} w .
\end{aligned}
$$

According to (1.9), we have

$$
\begin{aligned}
\widehat{f}_{\mathbb{H}}(\widehat{w})-\delta_{n, m} & \int_{\mathbb{H}^{d}} f(w) \mathrm{d} w \\
= & \int_{\mathbb{H}^{d}} f(w)\left(e^{-i \lambda(s+2\langle\eta, y\rangle)} \int_{\mathbb{R}^{d}} e^{-2 i \lambda\langle\eta, z\rangle} H_{n, \lambda}(z+2 y) H_{m, \lambda}(z) \mathrm{d} z\right. \\
& \left.\quad-\int_{\mathbb{R}^{d}} H_{n, \lambda}(z) H_{m, \lambda}(z) \mathrm{d} z\right) \mathrm{d} w .
\end{aligned}
$$

The right-hand side may be decomposed into $I_{1}+I_{2}+I_{3}$ with

$$
\begin{aligned}
I_{1} & =\int_{\mathbb{H}^{d}} e^{-i \lambda(s+2\langle\eta, y\rangle)} f(w)\left(\int_{\mathbb{R}^{d}}\left(e^{-2 i \lambda\langle\eta, z\rangle}-1\right) H_{n, \lambda}(z+2 y) H_{m, \lambda}(z) \mathrm{d} z\right) \mathrm{d} w, \\
I_{2} & =\int_{\mathbb{H}^{d}} e^{-i \lambda(s+2\langle\eta, y\rangle)} f(w)\left(\int_{\mathbb{R}^{d}}\left(H_{n, \lambda}(z+2 y)-H_{n, \lambda}(z)\right) H_{m, \lambda}(z) \mathrm{d} z\right) \mathrm{d} w \\
\text { and } \quad I_{3} & =\int_{\mathbb{H}^{d}}\left(e^{-i \lambda(s+2\langle\eta, y\rangle)}-1\right) f(w)\left(\int_{\mathbb{R}^{d}} H_{n, \lambda}(z) H_{m, \lambda}(z) \mathrm{d} z\right) \mathrm{d} w .
\end{aligned}
$$

To bound $I_{1}$, it suffices to use that

$$
\begin{aligned}
& \left|\int_{\mathbb{R}^{d}}\left(e^{-2 i \lambda\langle\eta, z\rangle}-1\right) H_{n, \lambda}(z+2 y) H_{m, \lambda}(z) \mathrm{d} z\right| \\
& \quad \leqslant 2 \sum_{j=1}^{d}\left|\lambda \eta_{j}\right| \int_{\mathbb{R}^{d}}\left|H_{n, \lambda}(z+2 y)\right|\left|z_{j} H_{m, \lambda}(z)\right| \mathrm{d} z
\end{aligned}
$$


whence, combining Cauchy-Schwarz inequality and (A.4),

$$
\left|\int_{\mathbb{R}^{d}}\left(e^{-2 i \lambda\langle\eta, z\rangle}-1\right) H_{n, \lambda}(z+2 y) H_{m, \lambda}(z) \mathrm{d} z\right| \leqslant \sum_{j=1}^{d}\left|\eta_{j}\right| \sqrt{|\lambda|\left(4 m_{j}+2\right)} .
$$

This gives

$$
\left|I_{1}\right| \leqslant \sqrt{|\lambda|(4|m|+2 d)} \int_{\mathbb{H}^{d}}|\eta||f(y, \eta, s)| \mathrm{d} y \mathrm{~d} \eta \mathrm{d} s .
$$

To handle the term $I_{2}$, we use the following mean value formula:

$$
H_{n, \lambda}(z+2 y)-H_{n, \lambda}(z)=2 y \cdot \int_{0}^{1} \nabla H_{n, \lambda}(z+2 t y) \mathrm{d} t,
$$

which implies, still using (A.4),

$$
\left|\int_{\mathbb{R}^{d}}\left(H_{n, \lambda}(z+2 y)-H_{n, \lambda}(z)\right) H_{m, \lambda}(z) \mathrm{d} z\right| \leqslant \sum_{j=1}^{d}\left|y_{j}\right| \sqrt{|\lambda|\left(4 n_{j}+2\right)},
$$

and thus

$$
\left|I_{2}\right| \leqslant \sqrt{|\lambda|(4|n|+2 d)} \int_{\mathbb{H}^{d}}|y||f(y, \eta, s)| \mathrm{d} y \mathrm{~d} \eta \mathrm{d} s .
$$

Finally, it is clear that the mean value theorem (for the exponential function) and the fact that $\left(H_{n}\right)_{n \in \mathbb{N}^{d}}$ is an orthonormal family imply that

$$
\left|I_{3}\right| \leqslant|\lambda| \delta_{n, m} \int_{\mathbb{H}^{d}}|s+2\langle\eta, y\rangle||f(y, \eta, s)| \mathrm{d} y \mathrm{~d} \eta \mathrm{d} s .
$$

Putting (5.2), (5.3) and (5.4) together ends the proof of the lemma.

Conclusion of the proof of Proposition 2.15. - Taking $\lambda= \pm \frac{\lambda^{\prime}}{2|n|+d}$ in Lemma 5.2, we discover that

$$
\left|\theta\left(n, n, \frac{\lambda^{\prime}}{2|n|+d}\right)+\theta\left(n, n,-\frac{\lambda^{\prime}}{2|n|+d}\right)-2 \theta(\widehat{0})\right| \leqslant C\|\theta\|_{k, k, \mathcal{S}\left(\widehat{\mathbb{H}}^{d}\right)} \sqrt{\lambda^{\prime}} .
$$

This implies that

$$
\begin{aligned}
\mathcal{I}_{2} & \leqslant C\|\theta\|_{k, k, \mathcal{S}\left(\widehat{\mathbb{H}}^{d}\right)} \sum_{n \in \mathbb{N}^{d}} \frac{1}{(2|n|+d)^{d+1}} \int_{0}^{1}\left|\lambda^{\prime}\right|^{d+\frac{1}{2}-\gamma} \mathrm{d} \lambda^{\prime} \\
& \leqslant C\|\theta\|_{k, k, \mathcal{S}\left(\widehat{\mathbb{H}}^{d}\right)} \int_{0}^{1}\left|\lambda^{\prime}\right|^{d+\frac{1}{2}-\gamma} \mathrm{d} \lambda^{\prime} .
\end{aligned}
$$

As $\gamma<d+3 / 2$, combining with (5.1) completes the proof of the proposition.

\section{Examples of computations of Fourier transforms}

The present sectionaims at pointing out a few examples of computations of Fourier transform that may be easily achieved within our approach. 
Let us start with Proposition 2.18. The first identity is easy to prove. Indeed, according to (1.10), we have

$$
\begin{aligned}
\left\langle\mathcal{F}_{\mathbb{H}^{H}}\left(\delta_{0}\right), \theta\right\rangle_{\mathcal{S}^{\prime}\left(\widehat{\mathbb{H}}^{d}\right) \times \mathcal{S}\left(\widehat{\mathbb{H}}^{d}\right)} & =\left\langle\delta_{0},{ }^{t} \mathcal{F}_{\mathbb{H}}(\theta)\right\rangle_{\mathcal{S}^{\prime}\left(\mathbb{H}^{d}\right) \times \mathcal{S}\left(\mathbb{H}^{d}\right)} \\
& =\int_{\widehat{\mathbb{H}}^{d}}\left(H_{m, \lambda} \mid H_{n, \lambda}\right)_{L^{2}} \theta(\widehat{w}) \mathrm{d} \widehat{w} .
\end{aligned}
$$

As $\left(H_{n, \lambda}\right)_{n \in \mathbb{N}}$ is an orthonormal basis of $L^{2}\left(\mathbb{R}^{d}\right)$, we get

$$
\left\langle\mathcal{F}_{\mathbb{H}}\left(\delta_{0}\right), \theta\right\rangle_{\mathcal{S}^{\prime}\left(\widehat{\mathbb{H}}^{d}\right) \times \mathcal{S}\left(\widehat{\mathbb{H}}^{d}\right)}=\sum_{n \in \mathbb{N}^{d}} \int_{\mathbb{R}} \theta(n, n, \lambda)|\lambda|^{d} \mathrm{~d} \lambda
$$

which is exactly the first identity.

In order to prove the second identity, we start again from the definition of the Fourier transform on $\mathcal{S}^{\prime}\left(\mathbb{H}^{d}\right)$, and get

$$
\left\langle\mathcal{F}_{\mathbb{H}}(\mathbb{1}), \theta\right\rangle_{\mathcal{S}^{\prime}\left(\widehat{\mathbb{H}}^{d}\right) \times \mathcal{S}\left(\widehat{\mathbb{H}}^{d}\right)}=\int_{\mathbb{H}^{d}}\left({ }^{t} \mathcal{F}_{\mathbb{H}} \theta\right)(w) \mathrm{d} w .
$$

Let us underline that because ${ }^{t} \mathcal{F}_{\mathbb{H}} \theta$ belongs to $\mathcal{S}\left(\mathbb{H}^{d}\right)$, the above integral makes sense. Besides, (2.15) implies that

$$
\left\langle\mathcal{F}_{\mathbb{H}}(\mathbb{1}), \theta\right\rangle_{\mathcal{S}^{\prime}\left(\widehat{\mathbb{H}}^{d}\right) \times \mathcal{S}\left(\widehat{\mathbb{H}}^{d}\right)}=\frac{\pi^{d+1}}{2^{d-1}} \int_{\mathbb{H}^{d}}\left(\mathcal{F}_{\mathbb{H}^{-1}}^{-1}(\theta)\right)(y,-\eta,-s) \mathrm{d} y \mathrm{~d} \eta \mathrm{d} s .
$$

By Theorem 2.7 and Lemma 5.2 we have, for any integrable function $f$ on $\mathbb{H}^{d}$,

$$
\widehat{f}_{\mathbb{H}}(\widehat{0})=\int_{\mathbb{H}^{d}} f(w) \mathrm{d} w
$$

Thus we get

$$
\left\langle\mathcal{F}_{\mathbb{H}}(\mathbb{1}), \theta\right\rangle_{\mathcal{S}^{\prime}\left(\widehat{\mathbb{H}}^{d}\right) \times \mathcal{S}\left(\widehat{\mathbb{H}}^{d}\right)}=\frac{\pi^{d+1}}{2^{d-1}} \mathcal{F}_{\mathbb{H}}\left(\mathcal{F}_{\mathbb{H}}^{-1}(\theta)\right)(\widehat{0})=\frac{\pi^{d+1}}{2^{d-1}} \theta(\widehat{0}) .
$$

This concludes the proof of the proposition.

In order to prove Theorem 2.19, we need the following obvious property of the Fourier transform.

Lemma 6.1. - Let $\left(T_{n}\right)_{n \in \mathbb{N}}$ be a sequence of tempered distribution on $\mathbb{H}^{d}$ which converges to $T$ in $\mathcal{S}^{\prime}\left(\mathbb{H}^{d}\right)$. Then the sequence $\left(\mathcal{F}_{\mathbb{H}} T_{n}\right)_{n \in \mathbb{N}}$ converges to $\mathcal{F}_{\mathbb{H}} T$ in $\mathcal{S}^{\prime}\left(\widehat{\mathbb{H}}^{d}\right)$.

Now, proving Theorem 2.19 just amounts to recasting Theorem 1.4 of [BCD] (and its proof) in terms of tempered distributions. We recall here the statement for the reader convenience.

Theorem 6.2. - Let $\chi$ be a function of $\mathcal{S}(\mathbb{R})$ with value 1 at 0 , and compactly supported Fourier transform. Then for any function $g$ in $L^{1}\left(T^{\star} \mathbb{R}^{d}\right)$ and any sequence $\left(\varepsilon_{n}\right)_{n \in \mathbb{N}}$ of positive real numbers tending to 0 , we have

$$
\lim _{n \rightarrow \infty} \mathcal{F}_{\mathbb{H}}\left(g \otimes \chi\left(\varepsilon_{n} \cdot\right)\right)=2 \pi\left(\mathcal{G}_{\mathbb{H}} g\right) \mu_{\widehat{\mathbb{H}}_{0}^{d}}
$$

in the sense of measures on $\widehat{\mathbb{H}}^{d}$. 
Proof of Theorem 2.19. - As $g \otimes \chi\left(\varepsilon_{n} \cdot\right)$ tends to $g \otimes \mathbb{1}$ in $\mathcal{S}^{\prime}\left(\mathbb{H}^{d}\right)$, Proposition 6.1 ensures that

$$
\mathcal{F}_{\mathbb{H}}(g \otimes \mathbb{1})=\lim _{n \rightarrow \infty} \mathcal{I}_{\varepsilon_{n}}(g, \theta) \quad \text { with } \quad \mathcal{I}_{\varepsilon_{n}}(g, \theta) \stackrel{\text { def }}{=} \mathcal{F}_{\mathbb{H}}\left(g \otimes \chi\left(\varepsilon_{n} \cdot\right)\right) .
$$

Moreover, according to Theorem 6.2 , we have, for any $\theta$ in $\mathcal{S}\left(\widehat{\mathbb{H}}^{d}\right)$,

$$
\mathcal{I}_{\varepsilon_{n}}(g, \theta)=\int_{\widehat{\mathbb{H}}^{d}} \frac{1}{\varepsilon_{n}} \widehat{\chi}\left(\frac{\lambda}{\varepsilon_{n}}\right) G(\widehat{w}) \theta(\widehat{w}) \mathrm{d} \widehat{w} \quad \text { with } \quad G(\widehat{w}) \stackrel{\text { def }}{=} \int_{T^{\star} \mathbb{R}^{d}} \overline{\mathcal{W}}(\widehat{w}, Y) g(Y) \mathrm{d} Y .
$$

As $g$ is integrable on $T^{\star} \mathbb{R}^{d}$, Proposition 2.1 of $[\mathrm{BCD}]$ implies that the (numerical) product $G \theta$ is a continuous function that satisfies

$$
|G(\widehat{w}) \theta(\widehat{w})| \leqslant C(1+|\lambda|(|n+m|+d)+|n-m|)^{-2 d+1} .
$$

This matches the hypothesis of Lemma 3.1 in $[\mathrm{BCD}]$, and thus

$$
\lim _{n \rightarrow \infty} \int_{\widehat{\mathbb{H}}^{d}} \frac{1}{\varepsilon_{n}} \widehat{\chi}\left(\frac{\lambda}{\varepsilon_{n}}\right) G(\widehat{w}) \theta(\widehat{w}) \mathrm{d} \widehat{w}=\int_{\widehat{\mathbb{H}}_{0}^{d}} \theta(\dot{x}, k)\left(\mathcal{G}_{\mathbb{H}} g\right)(\dot{x}, k) \mathrm{d} \mu_{\widehat{\mathbb{H}}_{0}^{d}}(\dot{x}, k) .
$$

Together with (6.3), this proves the theorem.

\section{Appendix A. Useful tools and more results}

For the reader's convenience, we here recall (and sometimes prove) some results that have been used repeatedly in the paper. We also provide one more result concerning the action of the Fourier transform on derivatives.

\section{A.1. Hermite functions}

In addition to the creation operator $C_{j} \stackrel{\text { def }}{=}-\partial_{j}+M_{j}$ already defined in the introduction, we used the following annihilation operator:

$$
A_{j} \stackrel{\text { def }}{=} \partial_{j}+M_{j}
$$

It is very classical (see e.g. [Olv74]) that

$$
A_{j} H_{n}=\sqrt{2 n_{j}} H_{n-\delta_{j}} \text { and } C_{j} H_{n}=\sqrt{2 n_{j}+2} H_{n+\delta_{j}} .
$$

As, obviously,

$$
2 M_{j}=C_{j}+A_{j} \text { and } 2 \partial_{j}=A_{j}-C_{j},
$$

we discover that

$$
\begin{aligned}
M_{j} H_{n} & =\frac{1}{2}\left(\sqrt{2 n_{j}} H_{n-\delta_{j}}+\sqrt{2 n_{j}+2} H_{n+\delta_{j}}\right) \\
\text { and } \partial_{j} H_{n} & =\frac{1}{2}\left(\sqrt{2 n_{j}} H_{n-\delta_{j}}-\sqrt{2 n_{j}+2} H_{n+\delta_{j}}\right) .
\end{aligned}
$$




\section{A.2. The inversion theorem}

We here present the proof of Theorem 1.3. In order to establish the inversion formula, consider a function $f$ in $\mathcal{S}\left(\mathbb{H}^{d}\right)$. Then we observe that if we make the change of variable $x^{\prime}=x-2 y$ in the integral defining $\left(\mathfrak{F}_{\mathbb{H}}(f)(\lambda)(u)\right)(x)$ and use the definition of the Fourier transform with respect to the variable $s$ in $\mathbb{R}$, then we get

$$
\begin{aligned}
\left(\mathfrak{F}_{\mathbb{H}}(f)(\lambda)(u)\right)(x) & =\int_{\mathbb{H}^{d}} f(y, \eta, s) e^{-i \lambda s-2 i \lambda\langle\eta, x-y\rangle} u(x-2 y) \mathrm{d} y \mathrm{~d} \eta \mathrm{d} s \\
& =2^{-d} \int_{T^{\star} \mathbb{R}^{d}}\left(\mathcal{F}_{s} f\right)\left(\frac{x-x^{\prime}}{2}, \eta, \lambda\right) e^{-i \lambda\left\langle\eta, x+x^{\prime}\right\rangle} u\left(x^{\prime}\right) \mathrm{d} x^{\prime} \mathrm{d} \eta .
\end{aligned}
$$

This can be written

$$
\begin{gathered}
\mathfrak{F}_{\mathbb{H}}(f)(\lambda)(u)(x)=\int_{\mathbb{R}^{d}} K_{f}\left(x, x^{\prime}, \lambda\right) u\left(x^{\prime}\right) \mathrm{d} x^{\prime}, \\
\text { with } \quad K_{f}\left(x, x^{\prime}, \lambda\right) \stackrel{\text { def }}{=} 2^{-d} \int_{\left(\mathbb{R}^{d}\right)^{\star}}\left(\mathcal{F}_{s} f\right)\left(\frac{x-x^{\prime}}{2}, \eta, \lambda\right) e^{-i \lambda\left\langle\eta, x+x^{\prime}\right\rangle} \mathrm{d} \eta \\
=2^{-d}\left(\mathcal{F}_{\eta, s} f\right)\left(\frac{x-x^{\prime}}{2}, \lambda\left(x+x^{\prime}\right), \lambda\right) .
\end{gathered}
$$

This identity enables us to decompose $\mathcal{F}_{\mathbb{H}}$ into the product of three very simple operations, namely

$$
\begin{gathered}
\mathcal{F}_{\mathbb{H}}=2^{-d} P_{H} \circ \Phi \circ \mathcal{F}_{\eta, s} \\
\text { with } \Phi(\phi)\left(x, x^{\prime}, \lambda\right) \stackrel{\text { def }}{=} \phi\left(\frac{x-x^{\prime}}{2}, \lambda\left(x+x^{\prime}\right), \lambda\right) \\
\text { and } \quad\left(P_{H} \psi\right)(n, m, \lambda) \stackrel{\text { def }}{=}\left(\psi(\cdot, \lambda) \mid H_{n, \lambda} \otimes H_{m, \lambda}\right)_{L^{2}\left(\mathbb{R}^{2 d}\right)} .
\end{gathered}
$$

Let us point out that for all $\lambda$ in $\mathbb{R} \backslash\{0\}$, the map

$$
\phi(\cdot, \lambda) \longmapsto \Phi(\phi)(\cdot, \lambda)
$$

is an automorphism of $L^{2}\left(\mathbb{R}^{2 d}\right)$ such that

$$
\|\Phi(\phi)(\cdot, \lambda)\|_{L^{2}\left(\mathbb{R}^{2 d}\right)}=|\lambda|^{-\frac{d}{2}}\|\phi(\cdot, \lambda)\|_{L^{2}\left(\mathbb{R}^{2 d}\right)},
$$

and that the inverse of $\Phi$ is explicitly given by

$$
\Phi^{-1}(y, z, \lambda)=\psi\left(y+\frac{z}{2 \lambda},-y+\frac{z}{2 \lambda}, \lambda\right) .
$$

Next, Operator $P_{H}$ just associates to any vector of $L^{2}\left(\mathbb{R}^{2 d}\right)$ its coordinates with respect to the orthonormal basis $\left(H_{n, \lambda} \otimes H_{m, \lambda}\right)_{(n, m) \in \mathbb{N}^{2 d}}$. It is, by definition, an isometric isomorphism from $L^{2}\left(\mathbb{R}^{2 d}\right)$ to $\ell^{2}\left(\mathbb{N}^{2 d}\right)$, with inverse

$$
\left(P_{H}^{-1} \theta\right)\left(x, x^{\prime}, \lambda\right)=\sum_{(n, m) \in \mathbb{N}^{2 d}} \theta(n, m, \lambda) H_{n, \lambda}(x) H_{m, \lambda}\left(x^{\prime}\right) .
$$

Obviously, arguing by density, Formula (A.7) may be extended to $L^{2}\left(\mathbb{H}^{d}\right)$. Therefore, according to Identities (A.8)-(A.10) and to the classical Fourier-Plancherel 
theorem in $\mathbb{R}^{d+1}$, the Fourier transform $\mathcal{F}_{\mathbb{H}}$ may be seen as the composition of three invertible and bounded operators on $L^{2}$, and we have

$$
\mathcal{F}_{\mathbb{H}}^{-1}=2^{d} \mathcal{F}_{\eta, s}^{-1} \circ \Phi^{-1} \circ P_{H}^{-1} .
$$

This gives (1.11) and (1.12). For the proof of (1.13), we refer for instance to [BCD]. This concludes the proof of Theorem 1.3.

\section{A.3. Properties related to the sub-ellipticity of the sublaplacian}

Under Notation (1.5), denote by $\mathcal{X}=\left(\mathcal{X}_{1}, \ldots, \mathcal{X}_{2 d}\right)$ the family of horizontal left invariant vector fields (where we agree that $\mathcal{X}_{j+d}=\Xi_{j}$ for $j$ in $\{1, \ldots, d\}$ ), and define, for any multi-index $\alpha$ in $\{1, \ldots, 2 d\}^{k}$ :

$$
\mathcal{X}^{\alpha} \stackrel{\text { def }}{=} \mathcal{X}_{\alpha_{1}} \ldots \mathcal{X}_{\alpha_{k}} .
$$

Then setting, for a nonnegative integer $k$

$$
\|u\|_{\dot{H}^{k}\left(\mathbb{H}^{d}\right)}^{2} \stackrel{\text { def }}{=} \sum_{\alpha \in\{1, \ldots, 2 d\}^{k}}\left\|\mathcal{X}^{\alpha} u\right\|_{L^{2}}^{2},
$$

we have the following well-known result (see the proof in e.g. [CCX93, Hör85]):

Theorem A.1. - For any positive integer $\ell$, we have for some constant $C_{\ell}>0$,

$$
\left\|\Delta_{\mathbb{H}}^{\ell} u\right\|_{L^{2}\left(\mathbb{H}^{d}\right)} \leqslant\|u\|_{\dot{H}^{2 \ell}\left(\mathbb{H}^{d}\right)} \leqslant C_{\ell}\left\|\Delta_{\mathbb{H}^{\ell}}^{\ell} u\right\|_{L^{2}\left(\mathbb{H}^{d}\right)} .
$$

This will enable us to establish the following proposition which states that the usual semi-norms on the Schwartz class and the semi-norms using the structure of $\mathbb{H}^{d}$ are equivalent.

Proposition A.2. - Let us introduce the notation

$$
\left(M_{\mathbb{H}} f\right)(X, s) \stackrel{\text { def }}{=}\left(|X|^{2}-i s\right) f(X, s) .
$$

Next, for all $\alpha=\left(\alpha_{0}, \alpha_{1}, \ldots, \alpha_{2 d}\right)$ in $\mathbb{N}^{1+2 d}$, we define

$$
w^{\alpha} \stackrel{\text { def }}{=} s^{\alpha_{0}} y_{1}^{\alpha_{1}} \ldots y_{d}^{\alpha_{d}} \eta_{1}^{\alpha_{d+1}} \ldots \eta_{d}^{\alpha_{2 d}} \text { and } \widetilde{|\alpha|} \stackrel{\text { def }}{=} 2 \alpha_{0}+\alpha_{1}+\ldots+\alpha_{2 d} .
$$

Then the two families of semi-norms defined on $\mathcal{S}\left(\mathbb{H}^{d}\right)$ by

$$
\begin{aligned}
&\|f\|_{p, \mathcal{S}\left(\mathbb{H}^{d}\right)}^{2} \stackrel{\text { def }}{=}\|f\|_{L^{2}}^{2}+\left\|M_{\mathbb{H}}^{p} f\right\|_{L^{2}}^{2}+\left\|\Delta_{\mathbb{H}}^{p} f\right\|_{L^{2}}^{2} \\
& \text { and } \quad N_{p}^{2}(f) \stackrel{\text { def }}{=} \sum_{|\alpha|+|\beta| \leqslant p}\left\|w^{\alpha} \mathcal{X}^{\beta} f\right\|_{L^{2}}^{2}
\end{aligned}
$$

are equivalent to the classical family of semi-norms on $\mathcal{S}\left(\mathbb{R}^{2 d+1}\right)$.

Proof. - As obviously $\|f\|_{p, \mathcal{S}\left(\mathbb{H}^{d}\right)} \leqslant N_{2 p}(f)$, showing that the two families of seminorms are equivalent reduces to proving that

$$
\forall p \in \mathbb{N}, \exists\left(C_{p}, M_{p}\right) / \forall f \in \mathcal{S}\left(\mathbb{H}^{d}\right), N_{p}(f) \leqslant C_{p}\|f\|_{M_{p}, \mathcal{S}\left(\mathbb{H}^{d}\right)} .
$$

Now, integrating by parts yields

$$
\int_{\mathbb{H}^{d}} w^{\alpha} \mathcal{X}^{\beta} f(w) w^{\alpha} \mathcal{X}^{\beta} \bar{f}(w) \mathrm{d} w=(-1)^{|\beta|} \int_{\mathbb{H}^{d}} f(w) \mathcal{X}^{\beta}\left(w^{2 \alpha} \mathcal{X}^{\beta} \bar{f}(w)\right) \mathrm{d} w .
$$


Observe that $\mathcal{X}^{\gamma} w^{\gamma^{\prime}}$ is either null or an homogeneous polynomial (with respect to the dilations (1.4)) of degree $\gamma^{\prime}-\gamma$, and equal to 0 if the length of $\gamma$ is greater than the length of $\gamma^{\prime}$. Thus, thanks to Leibniz's rule, we have

$$
\left[\mathcal{X}^{\beta}, w^{2 \alpha}\right] f(w)=\sum_{\substack{\tau \alpha^{\prime}|\leqslant 2| \alpha|-1\\| \beta^{\prime}|\leqslant| \beta \mid-1}} a_{\alpha, \alpha^{\prime}, \beta^{\prime}, \beta} w^{\alpha^{\prime}} \mathcal{X}^{\beta^{\prime}} f(w) .
$$

Hence we get that

$$
\int_{\mathbb{H}^{d}} f(w) \mathcal{X}^{\beta}\left(w^{2 \alpha} \mathcal{X}^{\beta} \bar{f}(w)\right) \mathrm{d} w=\sum_{\substack{\left|\alpha^{\prime}\right| \leqslant 2|\alpha| \\\left|\beta^{\prime}\right| \leqslant|\beta|}} a_{\alpha, \alpha^{\prime}, \beta, \beta^{\prime}} \int_{\mathbb{H}^{d}} w^{\alpha^{\prime}} f(w) \mathcal{X}^{\beta^{\prime}} \mathcal{X}^{\beta} \bar{f}(w) \mathrm{d} w .
$$

Thanks to Cauchy-Schwarz inequality and by definition of $M_{\mathbb{H}}$, we get, applying Theorem A.1 and taking $p$ large enough,

$$
\sum_{\substack{\tau \alpha^{\prime}|\leqslant 2| \alpha|| \beta^{\prime}|\leqslant| \beta \mid}} a_{\alpha, \alpha^{\prime}, \beta, \beta^{\prime}} \int_{\mathbb{H}^{d}} w^{\alpha^{\prime}} f(w) \mathcal{X}^{\beta^{\prime}} \mathcal{X}^{\beta} \bar{f}(w) \mathrm{d} w \leqslant C\left(\|f\|_{L^{2}}^{2}+\left\|M_{\mathbb{H}}^{p} f\right\|_{L^{2}}^{2}+\left\|\Delta_{\mathbb{H}}^{p} f\right\|_{L^{2}}^{2}\right) .
$$

This proves that the two families of semi-norms in the above statement are equivalent.

In order to establish that they are also equivalent to the classical family, one can observe that for all $j$ in $\{1, \ldots, d\}$,

$$
S=\frac{1}{4}\left[\Xi_{j}, \mathcal{X}_{j}\right], \quad \partial_{y_{j}}=\mathcal{X}_{j}-\frac{\eta_{j}}{2}\left(\Xi_{j} \mathcal{X}_{j}-\mathcal{X}_{j} \Xi_{j}\right) \quad \text { and } \quad \partial_{\eta_{j}}=\Xi_{j}+\frac{y_{j}}{2}\left(\Xi_{j} \mathcal{X}_{j}-\mathcal{X}_{j} \Xi_{j}\right),
$$

from which we easily infer that

$$
\widetilde{\|} f \|_{p, \mathcal{S}\left(\mathbb{R}^{2 d+1}\right)} \leqslant C N_{2 p}(f) \quad \text { with } \widetilde{\|} f\left\|_{p, \mathcal{S}\left(\mathbb{R}^{2 d+1}\right)}^{2} \stackrel{\text { def }}{=} \sum_{|\alpha|+|\beta| \leqslant p}\right\| x^{\alpha} \partial^{\beta} f \|_{L^{2}\left(\mathbb{R}^{2 d+1}\right)}^{2} .
$$

This ends the proof of the proposition.

\section{A.4. Derivations and multiplication in the frequency space}

In Section 2, we only considered the effect of the sublaplacian $\Delta_{\mathbb{H}}$ or of the derivation $\partial_{s}$ on Fourier transform. Those operations led to multiplication by $-4|\lambda|(2|m|+d)$ or $i \lambda$, respectively, of the Fourier transform. We also studied the effect of the multiplication by $|Y|^{2}$ or $-i s$, and found out that they correspond to the "derivation operators" $\widehat{\Delta}$ and $\widehat{\mathcal{D}}_{\lambda}$ for functions on $\widetilde{\mathbb{H}}^{d}$.

Our purpose here is to study the effect of left invariant differentiations $\mathcal{X}_{j}$ and $\Xi_{j}$ and multiplication by $M_{j}^{ \pm} \stackrel{\text { def }}{=} y_{j} \pm i \eta_{j}$ on the Fourier transform. This is described by the following proposition. 
Proposition A.3. - For any function $f$ in $\mathcal{S}\left(\mathbb{H}^{d}\right)$, we have, for $\lambda$ different from 0 ,

$$
\mathcal{F}_{\mathbb{H}} \mathcal{X}_{j} f=-\widehat{\mathcal{M}}_{j}^{+} \widehat{f}_{\mathbb{H}} \quad \text { and } \quad\left(\mathcal{F}_{\mathbb{H}} \Xi_{j} f\right)=-\widehat{\mathcal{M}}_{j}^{-} \widehat{f}_{\mathbb{H}}
$$

with $\widehat{\mathcal{M}}_{j}^{+} \theta(\widehat{w}) \stackrel{\text { def }}{=}|\lambda|^{\frac{1}{2}}\left(\sqrt{2 m_{j}+2} \theta\left(n, m+\delta_{j}, \lambda\right)-\sqrt{2 m_{j}} \theta\left(n, m-\delta_{j}, \lambda\right)\right)$

and $\widehat{\mathcal{M}}_{j}^{-} \theta(\widehat{w}) \stackrel{\text { def }}{=} \frac{i \lambda}{|\lambda|^{\frac{1}{2}}}\left(\sqrt{2 m_{j}+2} \theta\left(n, m+\delta_{j}, \lambda\right)+\sqrt{2 m_{j}} \theta\left(n, m-\delta_{j}, \lambda\right)\right)$.

We also have $\mathcal{F}_{\mathbb{H}} M_{j}^{ \pm} f=\widehat{\mathcal{D}}_{j}^{ \pm} \widehat{f}_{\mathbb{H}}$ with

$$
\begin{aligned}
\left(\widehat{\mathcal{D}}_{j}^{ \pm} \theta\right)(\widehat{w}) \stackrel{\text { def }}{=} \frac{1_{\{ \pm \lambda>0\}}}{2|\lambda|^{\frac{1}{2}}}\left(\sqrt{2 n_{j}} \theta\left(n-\delta_{j}, m, \lambda\right)-\sqrt{2 m_{j}+2} \theta\left(n, m+\delta_{j}, \lambda\right)\right) \\
+\frac{1_{\{ \pm \lambda<0\}}}{2|\lambda|^{\frac{1}{2}}}\left(\sqrt{2 n_{j}+2} \theta\left(n+\delta_{j}, m, \lambda\right)-\sqrt{2 m_{j}} \theta\left(n, m-\delta_{j}, \lambda\right) .\right.
\end{aligned}
$$

Proof. — The main point is to compute

$$
\partial_{y_{j}} \mathcal{W}(\widehat{w}, Y), \quad \partial_{\eta_{j}} \mathcal{W}(\widetilde{w}, Y), \quad y_{j} \mathcal{W}(\widehat{w}, Y) \quad \text { and } \quad \eta_{j} \mathcal{W}(\widehat{w}, Y)
$$

By the definition of $\mathcal{W}$ and Leibniz's rule, we have, using the notation $f_{\lambda}(x) \stackrel{\text { def }}{=}$ $f\left(|\lambda|^{1 / 2} x\right)$,

$$
\begin{aligned}
\partial_{y_{j}} \mathcal{W}(\widehat{w}, Y)=\int_{\mathbb{R}^{d}} e^{2 i \lambda\langle z, \eta\rangle}|\lambda|^{\frac{1}{2}}\left(\left(\partial_{j} H_{n}\right)_{\lambda}(y+z) H_{m, \lambda}(-y+z)\right. & \\
& \left.-H_{n, \lambda}(y+z)\left(\partial_{j} H_{m}\right)_{\lambda}(-y+z)\right) \mathrm{d} z .
\end{aligned}
$$

From (A.4), we infer that

$$
\begin{aligned}
\partial_{y_{j}} \mathcal{W}(\widehat{w}, Y)= & \frac{|\lambda|^{\frac{1}{2}}}{2}\left(\sqrt{2 n_{j}} \mathcal{W}\left(n-\delta_{j}, m, \lambda, Y\right)\right)-\sqrt{2 n_{j}+2} \mathcal{W}\left(n+\delta_{j}, m, \lambda, Y\right) \\
& \left.-\sqrt{2 m_{j}} \mathcal{W}\left(n, m-\delta_{j}, \lambda, Y\right)+\sqrt{2 m_{j}+2} \mathcal{W}\left(n, m+\delta_{j}, \lambda, Y\right)\right)
\end{aligned}
$$

Let us observe that

$$
\begin{aligned}
\partial_{\eta_{j}} \mathcal{W}(\widehat{w}, Y) & =\int_{\mathbb{R}^{d}} 2 i \lambda z_{j} e^{2 i \lambda\langle\eta, z\rangle} H_{n, \lambda}(y+z) H_{m, \lambda}(-y+z) \mathrm{d} z \\
= & i \lambda \int_{\mathbb{R}^{d}} e^{2 i \lambda\langle\eta, z\rangle}\left(\left(y_{j}+z_{j}\right) H_{n, \lambda}(y+z) H_{m, \lambda}(-y+z)\right. \\
& \left.+H_{n, \lambda}(y+z)\left(-y_{j}+z_{j}\right) H_{m, \lambda}(-y+z)\right) \mathrm{d} z \\
= & \frac{i \lambda}{|\lambda|^{\frac{1}{2}}} \int_{\mathbb{R}^{d}} e^{2 i \lambda\langle\eta, z\rangle}\left(\left(M_{j} H_{n}\right)_{\lambda}(y+z) H_{m, \lambda}(-y+z)\right. \\
& \left.+H_{n, \lambda}(y+z)\left(M_{j} H_{m}\right)_{\lambda}(-y+z)\right) \mathrm{d} z .
\end{aligned}
$$

Now, using again (A.4), we get

$$
\begin{aligned}
\partial_{\eta_{j}} \mathcal{W}(\widehat{w}, Y) & =\frac{i \lambda}{2|\lambda|^{\frac{1}{2}}}\left(\sqrt{2 n_{j}} \mathcal{W}\left(n-\delta_{j}, m, \lambda, Y\right)\right)+\sqrt{2 n_{j}+2} \mathcal{W}\left(n+\delta_{j}, m, \lambda, Y\right) \\
& \left.+\sqrt{2 m_{j}} \mathcal{W}\left(n, m-\delta_{j}, \lambda, Y\right)+\sqrt{2 m_{j}+2} \mathcal{W}\left(n, m+\delta_{j}, \lambda, Y\right)\right) .
\end{aligned}
$$


For multiplication by $y_{j}$, we proceed along the same lines. By definition of $\mathcal{W}$, we have

$$
\begin{aligned}
y_{j} \mathcal{W}(\widehat{w}, Y)=\frac{1}{2|\lambda|^{\frac{1}{2}}} \int_{\mathbb{R}^{d}} e^{2 i \lambda\langle\eta, z\rangle}\left(\left(M_{j} H_{n}\right)_{\lambda}(y+z) H_{m, \lambda}(-y+z)\right. \\
\left.\quad-H_{n, \lambda}(y+z)\left(M_{j} H_{m}\right)_{\lambda}(-y+z)\right) \mathrm{d} z .
\end{aligned}
$$

Still using (A.4), we deduce that

$$
\begin{aligned}
(\mathrm{A} .16) y_{j} \mathcal{W}(\widehat{w}, Y)= & \frac{1}{4|\lambda|^{\frac{1}{2}}}\left(\sqrt{2 n_{j}} \mathcal{W}\left(n-\delta_{j}, m, \lambda, Y\right)\right)+\sqrt{2 n_{j}+2} \mathcal{W}\left(n+\delta_{j}, m, \lambda, Y\right) \\
& \left.-\sqrt{2 m_{j}} \mathcal{W}\left(n, m-\delta_{j}, \lambda, Y\right)-\sqrt{2 m_{j}+2} \mathcal{W}\left(n, m+\delta_{j}, \lambda, Y\right)\right) .
\end{aligned}
$$

For the multiplication by $\eta_{j}$, let us observe that, performing an integration by parts, we can write

$$
\begin{aligned}
\eta_{j} \mathcal{W}(\widehat{w}, Y) & =\frac{1}{2 i \lambda} \int_{\mathbb{R}^{d}} \partial_{z_{j}}\left(e^{2 i \lambda\langle\eta, z\rangle}\right) H_{n, \lambda}(y+z) H_{m, \lambda}(-y+z) \mathrm{d} z \\
& =\frac{i}{2 \lambda} \int_{\mathbb{R}^{d}} e^{2 i \lambda\langle\eta, z\rangle} \partial_{z_{j}}\left(H_{n, \lambda}(y+z) H_{m, \lambda}(-y+z)\right) \mathrm{d} z .
\end{aligned}
$$

Leibniz's rule implies that

$$
\begin{aligned}
\eta_{j} \mathcal{W}(\widehat{w}, Y)=\frac{i|\lambda|^{\frac{1}{2}}}{2 \lambda} \int_{\mathbb{R}^{d}} e^{2 i \lambda\langle\eta, z\rangle}\left(\left(\partial_{j} H_{n}\right)_{\lambda}(y+z) H_{m, \lambda}(-y+z)\right. \\
\left.+H_{n, \lambda}(y+z)\left(\partial_{j} H_{m}\right)_{\lambda}(-y+z)\right) \mathrm{d} z .
\end{aligned}
$$

Using (A.4), we deduce that

$$
\begin{aligned}
(\mathrm{A} .17) \eta_{j} \mathcal{W}(\widehat{w}, Y)= & \frac{i|\lambda|^{\frac{1}{2}}}{4 \lambda}\left(\sqrt{2 n_{j}} \mathcal{W}\left(n-\delta_{j}, m, \lambda, Y\right)\right)-\sqrt{2 n_{j}+2} \mathcal{W}\left(n+\delta_{j}, m, \lambda, Y\right) \\
& \left.+\sqrt{2 m_{j}} \mathcal{W}\left(n, m-\delta_{j}, \lambda, Y\right)-\sqrt{2 m_{j}+2} \mathcal{W}\left(n, m+\delta_{j}, \lambda, Y\right)\right) .
\end{aligned}
$$

As $e^{-i s \lambda} \mathcal{X}_{j}\left(e^{i s \lambda} \mathcal{W}(\widehat{w}, Y)\right)=2 i \eta_{j} \lambda \mathcal{W}(\widehat{w}, Y)+\partial_{y_{j}} \mathcal{W}(\widehat{w}, Y)$, we infer from (A.14) and (A.17) that

$$
\begin{aligned}
e^{-i s \lambda} \mathcal{X}_{j}\left(e^{i s \lambda} \mathcal{W}(\widehat{w}, \cdot)\right) & =|\lambda|^{\frac{1}{2}}\left(\sqrt{2 m_{j}+2} \mathcal{W}\left(n, m+\delta_{j}, \lambda, \cdot\right)-\sqrt{2 m_{j}} \mathcal{W}\left(n, m-\delta_{j}, \lambda, \cdot\right)\right) \\
& =\mathcal{M}_{j}^{+} \mathcal{W}(\widehat{w}, \cdot) .
\end{aligned}
$$

Then, using the definition of $\mathcal{F}_{\mathbb{H}}$ completes the proof of the first item of the proposition. Indeed, we have for all $\widehat{w}$ in $\widetilde{\mathbb{H}}^{d}$,

$$
\begin{aligned}
\mathcal{F}_{\mathbb{H}} \mathcal{X}_{j} f(\widehat{w}) & =\int_{\mathbb{H}^{d}} e^{-i s \lambda} \overline{\mathcal{W}(\widehat{w}, Y)} \mathcal{X}_{j} f(Y, s) \mathrm{d} w \\
& =-\int_{\mathbb{H}^{d}} \mathcal{X}_{j}\left(e^{-i s \lambda} \overline{\mathcal{W}(\widehat{w}, Y)}\right) f(Y, s) \mathrm{d} w \\
& =-\int_{\mathbb{H}^{d}} e^{-i s \lambda} \overline{\left(\mathcal{M}_{j}^{+} \mathcal{W}\right)(Y, s)} f(Y, s) \mathrm{d} w=-\mathcal{M}_{j}^{+} \mathcal{F}_{\mathbb{H}} f(\widehat{w}) .
\end{aligned}
$$


For proving the second item, we start from the relation

$$
e^{-i s \lambda} \Xi_{j}\left(e^{i s \lambda} \mathcal{W}(\widehat{w}, Y)\right)=-2 i y_{j} \lambda \mathcal{W}(\widehat{w}, Y)+\partial_{\eta_{j}} \mathcal{W}(\widehat{w}, Y),
$$

and infer from (A.15) and (A.16) that

$$
\begin{aligned}
e^{-i s \lambda} \Xi_{j}\left(e^{i s \lambda} \mathcal{W}(\widehat{w}, \cdot)\right) & =\frac{i \lambda}{|\lambda|^{\frac{1}{2}}}\left(\sqrt{2 m_{j}} \mathcal{W}\left(n, m-\delta_{j}, \lambda, \cdot\right)+\sqrt{2 m_{j}+2} \mathcal{W}\left(n, m+\delta_{j}, \lambda, \cdot\right)\right) \\
& =\mathcal{M}_{j}^{-} \mathcal{W}(\widehat{w}, \cdot) .
\end{aligned}
$$

Finally, it is obvious that (A.16) and (A.17) give

$$
\begin{aligned}
&\left(y_{j} \pm i \eta_{j}\right) \mathcal{W}(\widehat{w}, Y)= \frac{1}{4|\lambda|^{\frac{1}{2}}}\left(\sqrt{2 n_{j}} \mathcal{W}\left(n-\delta_{j}, m, \lambda, Y\right)\right)+\sqrt{2 n_{j}+2} \mathcal{W}\left(n+\delta_{j}, m, \lambda, Y\right) \\
&-\sqrt{2 m_{j}} \mathcal{W}\left(n, m-\delta_{j}, \lambda, Y\right)-\sqrt{2 m_{j}+2} \mathcal{W}\left(n, m+\delta_{j}, \lambda, Y\right) \\
& \pm \operatorname{sgn}(\lambda)\left(\sqrt{2 n_{j}} \mathcal{W}\left(n-\delta_{j}, m, \lambda, Y\right)-\sqrt{2 n_{j}+2} \mathcal{W}\left(n+\delta_{j}, m, \lambda, Y\right)\right. \\
&\left.\left.+\sqrt{2 m_{j}} \mathcal{W}\left(n, m-\delta_{j}, \lambda, Y\right)-\sqrt{2 m_{j}+2} \mathcal{W}\left(n, m+\delta_{j}, \lambda, Y\right)\right)\right) .
\end{aligned}
$$

Then reverting to the definition of $\mathcal{F}_{\mathbb{H}}$ gives the last part of the proposition.

\section{BIBLIOGRAPHY}

[ADBR13] Francesca Astengo, Bianca Di Blasio, and Fulvio Ricci, Fourier transform of Schwartz functions on the Heisenberg group, Stud. Math. 214 (2013), 201-222. $\uparrow 3$

[BCD] Hajer Bahouri, Jean-Yves Chemin, and Raphaël Danchin, A frequency space for the Heisenberg group, to appear in Ann. Inst. Fourier. $\uparrow 3,4,7,9,12,14,37,38,40$

[BFKG12] Hajer Bahouri, Clotilde Fermanian-Kammerer, and Isabelle Gallagher, Phase-space analysis and pseudodifferential calculus on the Heisenberg group, Astérisque, vol. 342, Société Mathématique de France, 2012. $\uparrow 3$

[CCX93] C. E. Cancelier, Jean-Yves Chemin, and Chao-Jiang Xu, Calcul de Weyl-Hörmander et opérateurs sous-elliptiques, Ann. Inst. Fourier 43 (1993), no. 4, 1157-1178. $\uparrow 40$

[Far10] Jacques Faraut, Asymptotic spherical analysis on the Heisenberg group, Colloq. Math. 118 (2010), no. 1, 233-258. $\uparrow 3$

[FH84] Jacques Faraut and Khélif Harzallah, Deux cours d'analyse harmonique, Progress in Mathematics, vol. 69, Birkhäuser, 1984. $\uparrow 3$

[Fol89] Gerald B. Folland, Harmonic Analysis in Phase Space, Annals of Mathematics Studies, vol. 122, Princeton University Press, 1989. $\uparrow 3$

[FR14] Véronique Fischer and Michael Ruzhansky, A pseudo-differential calculus on graded nilpotent Lie groups, Fourier analysis. Pseudo-differential operators, time-frequency analysis and partial differential equation, Trends in Mathematics, Birkhäuser, 2014, pp. 107-132. $\uparrow 3$

[FS82] Gerald B. Folland and Elias M. Stein, Hardy spaces on homogeneous groups, Mathematical Notes, vol. 28, Princeton University Press, 1982. $\uparrow 3$

[Gav77] Bernard Gaveau, Principe de moindre action, propagation de la chaleur et estimées sous-elliptiques sur certains groupes nilpotents, Acta Math. 139 (1977), 95-153. 110

[Gel77] Daryl Geller, Fourier analysis on the Heisenberg group, Proc. Natl. Acad. Sci. USA 74 (1977), 1328-1331. $\uparrow 3$ 
[Gel80] - Fourier analysis on the Heisenberg group I. the Schwartz space, J. Funct. Anal. 36 (1980), 205-254. $\uparrow 3$

[Hör85] Lars Hörmander, The analysis of linear partial differential operators. III: Pseudodifferential operators, Grundlehren der Mathematischen Wissenschaften, vol. 274, Springer, 1985. $\uparrow 40$

[Hue76] Denise Huet, Décomposition spectrale et opérateurs, Le Mathématicien, vol. 16, Presses Universitaires de France, 1976. $\uparrow 5$

[Hul84] Andrzej Hulanicki, A functional calculus for Rockland operators on nilpotent Lie groups, Stud. Math. 78 (1984), 253-266. $\uparrow 10$

[LT14] R. Lakshmi Lavanya and Sundaram Thangavelu, Revisiting the Fourier transform on the Heisenberg group, Publ. Mat., Barc. 58 (2014), 47-63. $\uparrow 3$

[Olv74] Frank W. J. Olver, Asymptotics and special functions, Computer Science and Applied Mathematics, Academic Press Inc., 1974. $\uparrow 5,38$

[Rud62] Walter Rudin, Fourier analysis on groups, Interscience Tracts in Pure and Applied Mathematics, vol. 12, Interscience Publishers, 1962. $\uparrow 2$

[Sch98] Laurent Schwartz, Théorie des distributions, Hermann, 1998. $\uparrow 2$

[Ste93] Elias M. Stein, Harmonic analysis: Real-variable methods, orthogonality, and oscillatory integrals, Princeton Mathematical Series, vol. 43, Princeton University Press, 1993. $\uparrow 3$

[Tay86] Michael E. Taylor, Noncommutative Harmonic Analysis, Mathematical Surveys and Monographs, vol. 22, American Mathematical Society, 1986. ^3, 4

[Tha98] Sundaram Thangavelu, Harmonic analysis on the Heisenberg group, Progress in Mathematics, vol. 159, Birkhäuser, 1998. $\uparrow 3$

Manuscript received on 13th December 2017, revised on 22nd December 2018, accepted on 24th December 2018.

Recommended by Editor F. Hérau. Published under license CC BY 4.0.

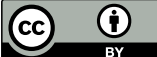

This journal is a member of Centre Mersenne.

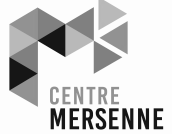

\author{
Hajer BAHOURI \\ LAMA, UMR 8050 \\ Université Paris-Est Créteil \\ 94010 Créteil Cedex (France) \\ hajer.bahouri@math.cnrs.fr \\ Jean-Yves CHEMIN \\ Laboratoire J.-L. Lions, UMR 7598 \\ Sorbonne Université \\ 75230 Paris Cedex 05 (France) \\ chemin@ann.jussieu.fr
}




\author{
Raphaël DANCHIN \\ LAMA, UMR 8050 \\ Université Paris-Est Créteil \\ 94010 Créteil Cedex (France) \\ danchin@u-pec.fr
}

